Uchasum, vel, 23

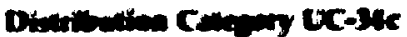

\title{
Docuana.
}

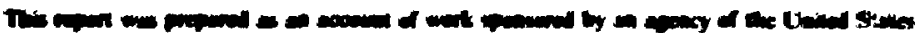

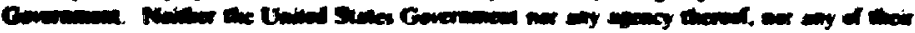

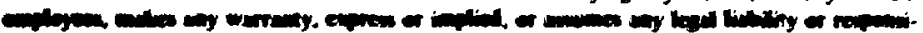

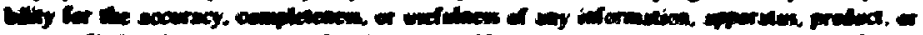

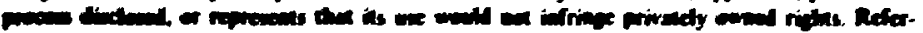

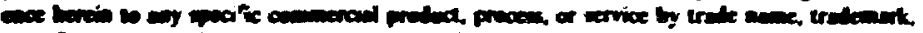

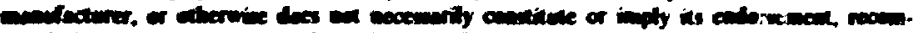

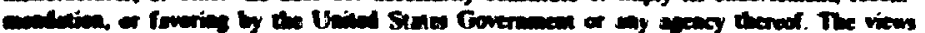

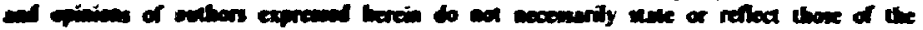

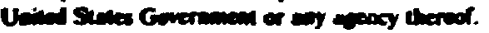

$001-50400-501.29$

D:37 003000

\section{Calculated Photon Kerma Factors Based on the LLNL EGDL Data File}

\author{
R. J. Howerton
}

Manuscript date: October 10, 1986

\section{MASTER}

\section{LAWRENCE LIVERMORE NATIONAL LABORATORY \\ University of California - Livermore, California - 94550}




\section{Comients}

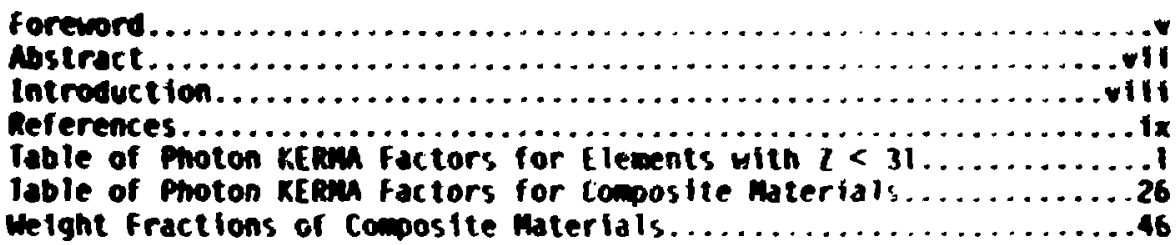




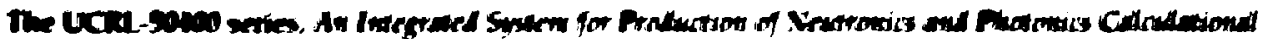

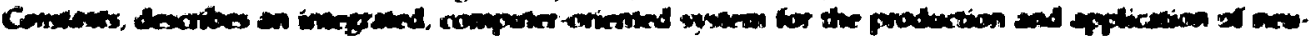

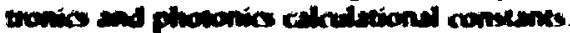

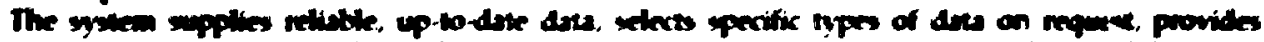

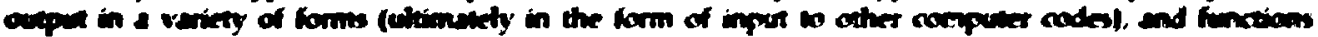
repinty and efficiemtly.

The UChL-50w0 series comprives the following volumes:

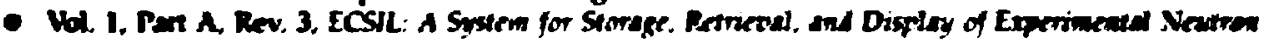
Date. Seprember 1976.

- Wol. 1. Part B. Program ECSX, Nerston 78-11: Conzersion of Experimentally Measured Cross-Section Dete from the Four-Center-Exchange $\alpha-4$ Format to the Livermore ECSIL Format, December 1578.

- Vol. 2, Rev. 2, A Bibliography of the Experimental Date of Neutron-Induced Interactions, July 1976.

- Vol. 3, Rev. 2, An Index of the Experimental Data of Neutron-Induced Interactions, July 1976.

- Vol. 4, Rev. 1. Evaluated Nuclear Dasa Librery. September 1981.

- Vol. 4, Rev. 1. Appendix C. The Neutron Library ENDL8y in the Transmitral Format, June 1982.

- Vol. 5. Part A, Rev. 1, CLYDE: A Code for the Production of Calculational Constents from Nuctear Data, September 1975.

- Vol. 5. Part B, Rev. 1, Relativistic Transformations betuceen Center-of-Mass and Laboratory Systems for Tzoo-Body Nuclear Reactions, April 1978.

- Vol. 6, Rev. 2, Tables and Graphs of Photon-Interaction Cross Sections from $1 \mathrm{keV}$ to $100 \mathrm{MeV}$, December 1978.

- Vol. 7. Part A, Rev. 1, Major Neutron-Induced Interactions $(Z \leq 55)$ : Graphical, Experimental Data, July 1976.

- Vol. 7, Part B, Rev. 1, Major Neutron-Induced Interactions $(Z>55$ ): Graphical, Experimental Data, July 1976.

- Vol. 8, Part A, Rev. 1, Supplemental Neutron-Induced Interactions $Z \leq 35$ ): Graphical, Experimental Data, July 1976.

- Vol. 8, Part B, Rev. 1, Supplemental Neutron-Induced Interactions $(Z>35)$ : Graphical, Experinental Data, July 1976.

- Vol. 9, Thresholds of Nuclear Reactions Induced by Neutrons, Photons, Deuterons, Tritons, and Alpha Particles, September 1970.

- Vol. 10, Rev. 1, Tabulated Experimental Data for Neutron-Induced Interactions, July 1976.

- Vol. 11, Experimental Data, Indexes, and Techniques of Obtaining a Selected Set of Neutron Resonance Parameters, May 1972.

- Vol. 12, An Atlas of Resolved Neutron Resonance Parameters, July 1972.

- Vol. 13, An Atlas of Unresolved Neutron Resonance Parameters, September 1972.

- Vol. 14, TARTNP: A Coupled Neutron-Photon Monte Carlo Transport Code, February 1976.

- Vol. 15, Part A, The iLL Evaluated-Nuclear-Data Library (ENDL): Evaluation Techniques, Reaction Index, and Descriptions of Individual Evaluations, September 1975.

- Vol. 15, Part B, Rev. 1, The LLL Evaluated-Nuclear-Data Library (ENDL): Graphs of Cross Sections from the Library, October 1978.

- Vol. 15, Part C, The LLL Evaluated-Nuclear-Data Library (ENDL): Translation of ENDL NeutronInduced Interaction Data into the ENDF/B Format, April 1976.

- Vol. 15, Part D, Rev. 1, The LLL Evaluated-Nuclear-Data Library (ENDL): Descriptions of Individual Evaluations for $Z=0-98$, May 1978.

- Vol. 15, Part E, Data Testing Results for the LLL Nuclear Data Library (ENDL-78), August 1979.

- Vol. 15, Part F, Experimental and Evaluated Elastic Nuclear Plus Interference Cross Sections for Light Charged Particles, July 1980.

- Vol. 16, Rev. 2, Tabular and Graphical Presentation of 175 Neutron-Group Constants Derived from the LLL Evaluated-Nuclear-Data Library (ENDL), October 1978.

- Vol. 17, Part A, Rev. 2, Program LINEAR (Version 79-1): Linearize Data in the Evaluated-NuclearData File/Version B (ENDF/F) Format, October 1979. 


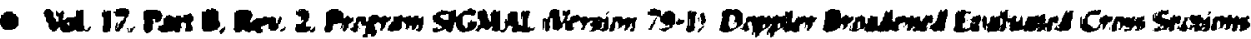

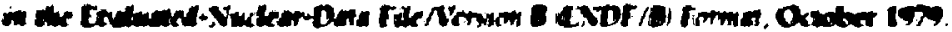

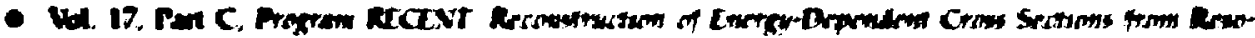

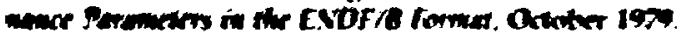

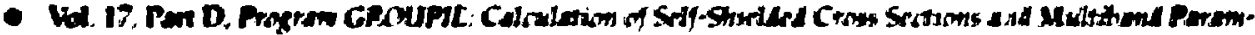

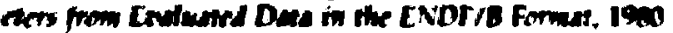

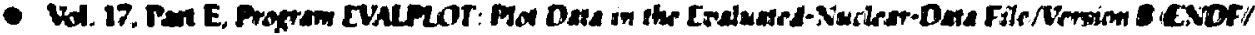
C) Forma, Fetruary 1979. 1979.

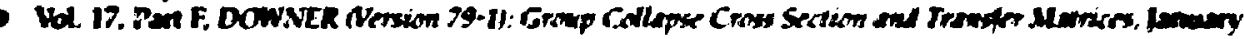

- Vol. 16. ACTL: Enduated Newtrsn Artization Cross-Sertion Litrary. October 1978.

- Vol. 19. Newtron-Induced Angular and Energy Distribufions: Graphical Experimented Data. April I97R.

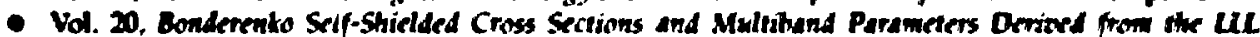
Evaluated-Nuclear-Dals Librany (ENDL. July 1978.

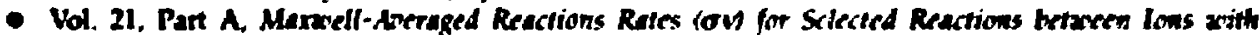
Atomic Mass $\leq 11$. February 1979.

- Vol. 21. Part C. Program SIGMAL Nersion 79-1): Doppler-Brondened Eoalwated Cross Sections in the Livermare-Evaluated Nuclear Data Library (ENDU Format, March 1979.

- Vol. 22, Rev. 1, GAMIDEN: A Program to Aid in the ldentificasion of Unknozen Materials by GanneaRay Spectroscopy, June 1982.

- Vol. 23, ENSL and CDRL: Evaluated Nuclear Structure Libraries, February 1981.

- Vol. 23, Addendum, ENSL82 and CDRL82: The 1982 Version of Epeluated Nuclear Structure Libraries. Jantuary 1983.

- Vol. 24, Thresholds and Q Values of Nuclear Reactions Induced by Neutrons. Protons, Deuterons. Tritons, ${ }^{3} \mathrm{He}$ lons, Alpha Particles, and Pholons, March 1981.

- Vol. 25, OMEGA: A CRAY 1 Executioe Code for LLNL Nuclear Dala Libraries, August 1983.

- Vol. 26, A Bibliography and Index for Juclear Reactions Among Light Charged Particies, September 1984.

- Vol. 27, Calculated Neutron KERMA Factors Based on the LLNL ENDL Data File, January 1986.

- Vol. 28, Index to the LLNL Evaluated Charged-Particle Library (ECPL). March 1986. 


\section{Astruct}

Fhoton (Cane-Ray) term factors calculated fron the UWJ ECDL (Lvelunted Cana-hay Data Library) tile are tabalated for the elements fron $2-1$ to $2-30$ and for 15 composite enterials. The kenh factors are presented for 191 energy groups over the Incident photon energy range from 100 ev to $100 \mathrm{HF}$. 


\section{Imooctios}

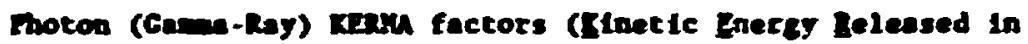
IMter) are used to assess phystologlcal danage Incurred by exposure to Gane-lays and to deternine heating in ater hals of interest from photon Interactions with ancer. The energy release is obtalned by formting the produce of the photon rum factor and the phocon florence at the approprlate photon energy. Conerelly, the rurh factor for a photon induced reaction 1s defined to be the energy avallable less the enercy cerried off by secondary photons. The kearh factor for a aterial is then obtained by swing the wexh factors of the Individual reactions, properly weighted by elenental abundance in the case of composte atterials.

In the context of this report, only photon interactions with atoas and wlecules are considered. The en $\times$ rgy deposited fros photo-nuclear reactions is not included. Thus, for photon energles greater than the least threshold of a photo-nuclear reaction of a naturally occurring isotope of an elenent, the values presented here will not include all contributions to the photon XERTA factors. This deficiency is caused by the lack of evaluated data needed to include the photo-nuclear phenomena in the calculated RERMA factors. An effort is currently underway to create a photo-nuclear data 11 brary so that future tabulations of photon XERYA factor can include contributions from such reactions. Since the least threshold of a photo-nuclear reaction occurs in the fer MeV region for naturaliy occurring 1sotopes, photon KERHA values presented here will be accurate for lesser energies. within the 1imitations of the data.

Explicit energy distributions for secondary photons are included in the EGDL (Evaluated Gamma-Ray Data LIbrary, See Ref. 1) for each reaction. From these distributions, average photon secondary energies are calculated and the result subtracted from the avallable energy to obtain the requisite energy for the reaction to calculate the RERMA factor. This value and all others for the various reactions are cross section weighted to obtain the photon-energy dependent KERHA factor for the element. For the composite materials, the RERMA factors for the individual elements are combined according to their weight fractions to obtain the value for the composite.

The tables of KERMA factors are presented for 191 energy groups from .0001 to $100 \mathrm{MeV}$. For photon energles between .001 and $20 \mathrm{MeV}$ the group boundarles correspond to the energles used by Singh (Ref, 2) so 


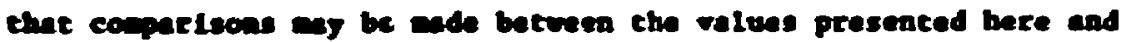
chose of inference 2.

The compontelons of the compostes aterials used here rere caken fron Caswli at al., (Ref. 3). The composition used hore differs s11phely Eron that of sineh (Ref. 2) for "scandard Man" which is the caly composite entertal given in comon.

2 The values presented in the tables are given in units of Gray $\mathrm{ca}^{2}$. The Grey is equivalenf to one Joule per $\mathrm{ks}$ or 100 rads or 10000 ergs per ge or $.624196 \times 10^{10}$ yeV per 8n.

\section{REFERELCES}

1. E.F. Plechaty, D.E. Cullen and R.J. Howerton, Tables and Graphs of Photon Interaction Gross Sections from 0.1 kev to 100 Hev Derfied fros the LLL Evaluated Puclear-Pete Ifbrery, UCRL-50400, Vo1. 6, Rev. 3, Lawrence LIvermore National Laboratory, 1981.

2. M.S. SIngh, XERYA EACTORS FOR NEUTRON AND PHOTONS WITH ENERGIES

BELOK 20 MeV. UCRL-52850, Lawrence Livermore Hational Laboratory, 1979.

3. R.S. Caswe11, J.J. Coyne., and M.L. Randolph, Rerma Factors for

Neutron Energles below 30 KeV, Radiation Research 83, 217-254 (1980). 
T11

Brey eninte

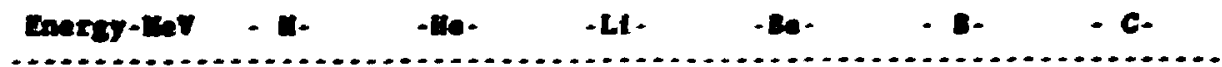

$1.0000-04$

$1.040 e-04 \quad 1.754 e-10 \quad 8.524 e-10 \quad 1.895 e-09 \quad 1.232 e-10 \quad 2.369 e-10 \quad 4.207 e-10$

$1.140 e-04 \quad 1.565 e-10 \quad \frac{225 e-10}{1.038 e-09} 1.457 e-092.312 e-10 \quad 4.099 e-10$

$1.360 e-041.104 e-10 \quad 7.246 e-10 \quad 1.645 e-09 \quad 2.631 e-09 \quad 2.103 e-10 \quad 3.715 e-10$

1.560e-04 $8.516 e-11 \quad 5.131 e-10 \quad 1.219 e-09 \quad 2.175 e-09 \quad 1.625 e-10 \quad 2.046 e-10$

$1.700 e-046.705 e-11 \quad 4.213 e-10 \quad 1.028 e-09 \quad 1.942 e-09 \quad 1.384 e-10 \quad 2.458 e-10$

$1.840 e-04 \quad 5.655 e-11 \quad 3.766 e-10 \quad 9.291 e-10 \quad 1.791 e-09 \quad 1.235 e-10 \quad 2.256 e-10$

$1.910 e-045.061 e-11 \quad 3.350 e-10 \quad 8.363 e-10 \quad 1.647 e-09 \quad 1.114 e-09$ 2.063e-10

2.140e-04 4.234e-11 2.819e-10 7.173e-10 1.458e-09 2.187e-09 1.811e-10

2.240e-04 3.710e-11 $2.625 e-10 \quad 6.735 e-10 \quad 1.383 e-09 \quad 2.091 e-09 \quad 1.759 e-10$

$2.410 e-04 \quad 3.256 e-11 \quad 2.488 e-10 \quad 6.420 e-10 \quad 1.324 e-09 \quad 2.028 e-09 \quad 1.630 e-10$

2.470e-04 2.815e-11 2.347e-10 6.092e-10 $1.263 e-09 \quad 1.959 e-09 \quad 1.545 e-10$

2.630e-04 2.505e-11 2.185e-10 5.713e-10 $1.191 e-09 \quad 1.875 e-09 \quad 1.452 e-10$

2.750e-04 2.298e-11 $1.950 e-10 \quad 5.156 e-10 \quad 1.085 e-09 \quad 1.749 e-09 \quad 1.312 e-10$

2.820e-04 2.144e-11 $1.768 e-10 \quad 4.728 e-10 \quad 1.003 e-09 \quad 1.650 e-09 \quad 1.204 e-10$

$2.960 e-04 \quad 1.953 e-11 \quad 1.546 e-10 \quad 4.200 e-10 \quad 9.023 e-10 \quad 1.526 e-09 \quad 1.897 e-09$

3.180e-04 1.679e-11 $1.277 e-10 \quad 3.556 e-10 \quad 7.784 e-10 \quad 1.369 e-09 \quad 2.002 e-09$

3.550e-04 1.376e-11 $1.142 e-10 \quad 3.216 e-10 \quad 7.114 e-10 \quad 1.263 e-09 \quad 1.885 e-09$

3.610e-04 $1.172 \mathrm{e}-11 \quad 1.025 e-10 \quad 2.920 e-10 \quad 6.522 e-10 \quad 1.166 e-09 \quad 1.775 e-09$

$3.700 e-04 \quad 1.128 e-11 \quad 9.778 e-11 \quad 2.800 e-10 \quad 6.280 e-10 \quad 1.127 e-09 \quad 1.728 e-09$

$3.830 e-04 \quad 1.058 e-11 \quad 9.035 e-11 \quad 2.610 e-10 \quad 5.895 e-10 \quad 1.063 e-09 \quad 1.654 e-09$

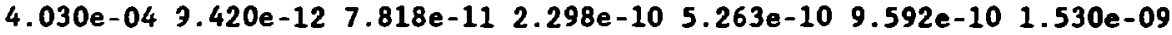

$4.150 e-04 \quad 8.581 e-12 \quad 7.081 e-11 \quad 2.107 e-10 \quad 4.869 e-10 \quad 8.939 e-10 \quad 1.448 e-09$

$4.300 e-048.088 e-12 \quad 6.800 e-11 \quad 2.032 e-10 \quad 4.704 e-10 \quad 8.663 e-10 \quad 1.409 e-09$

4.500e-04 7.375e-12 6.386e-11 $1.921 e-10 \quad 4.460 e-10 \quad 8.251 e-10 \quad 1.350 e-09$

$\begin{array}{lllllll}4.810 e-04 & 6.504 e-12 & 5.682 e-11 & 1.731 e-10 & 4.040 e-10 & 7.538 e-10 & 1.246 e-09\end{array}$

$5.160 e-04 \quad 5.509 e-12 \quad 4.693 e-11 \quad 1.462 e-10 \quad 3.445 e-10 \quad 6.522 e-10 \quad 1.096 e-09$

5.470e-04 4.725e-12 4.166e-11 $1.318 \mathrm{e}-10 \quad 3.127 \mathrm{e}-10 \quad 5.961 e-10 \quad 1.009 e-09$

$\begin{array}{lllllll}5.550 e-04 & 4.269 e-12 & 3.889 e-11 & 1.242 e-10 & 2.958 e-10 & 5.658 e-10 & 9.604 e-10\end{array}$

$\begin{array}{lllllll}5.930 e-04 & 3.937 e-12 & 3.515 e-11 & 1.138 e-10 & 2.728 e-10 & 5.242 e-10 & 8.935 e-10\end{array}$

$5.980 e-04 \quad 3.607 e-12 \quad 3.136 e-11 \quad 1.033 e-10 \quad 2.494 e-10 \quad 4.819 e-10 \quad 8.251 e-10$

$6.250 e-04 \quad 3.429 e-12 \quad 2.971 e-11 \quad 9.852 e-11 \quad 2.388 e-10 \quad 4.624 e-10 \quad 7.940 e-10$

$\begin{array}{lllllll}6.540 e-04 & 3.154 e-12 & 2.752 e-11 & 9.203 e-11 & 2.244 e-10 & 4.354 e-10 & 7.511 e-10\end{array}$

$\begin{array}{lllllll}6.750 e-04 & 2.914 e-12 & 2.532 e-11 & 8.547 e-11 & 2.097 e-10 & 4.078 e-10 & 7.072 e-10\end{array}$

$\begin{array}{lllllll}6.980 e-04 & 2.704 e-12 & 2.317 e-11 & 7.903 e-11 & 1.953 e-10 & 3.808 e-10 & 6.639 e-10\end{array}$

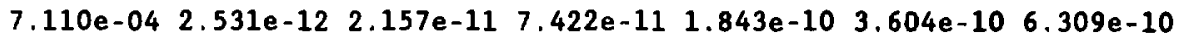

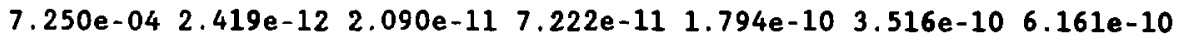

$\begin{array}{lllllll}7.710 e-04 & 2.166 e-12 & 1.929 e-11 & 6.732 e-11 & 1.673 e-10 & 3.302 e-10 & 5.795 e-10\end{array}$

$\begin{array}{lllllll}7.940 e-04 & 1.927 e-12 & 1.722 e-11 & 6.101 e-11 & 1.517 e-10 & 3.026 e-10 & 5.323 e-10\end{array}$

$8.180 e-04 \quad 1.787 e-12 \quad 1.589 e-11 \quad 5.696 e-11 \quad 1.417 e-10 \quad 2.846 e-10 \quad 5.017 e-10$

$8.380 e-04 \quad 1.697 e-12 \quad 1.512 e-11 \quad 5.453 e-11 \quad 1.358 e-10 \quad 2.737 e-10 \quad 4.835 e-10$

$\begin{array}{lllllll}8.600 e-04 & 1.610 e-12 & 1.435 e-11 & 5.210 e-11 & 1.299 e-10 & 2.626 e-10 & 4.651 e-10\end{array}$

8.980e-04 1.501e-12 1.314e-11 4.828e-11 $1.207 e-10 \quad 2.452 e-10 \quad 4.361 e-10$

$9.200 e-1441.387 e-12 \quad 1.210 e-11 \quad 4.438 e-11 \quad 1.119 e-10 \quad 2.287 e-10 \quad 4.095 e-10$

$9.400 e-1041.303 e-121.174 e-11 \quad 4.188 e-11 \quad 1.076 e-10 \quad 2.201 e-10 \quad 3.978 e-10$

$9.560 \mathrm{e}-04 \quad 1.230 \mathrm{e}-12 \quad 1.142 \mathrm{e}-11 \quad 3.961 \mathrm{e}-11 \quad 1.036 \mathrm{e}-10 \quad 2.123 \mathrm{e}-10 \quad 3.870 \mathrm{e}-10$ 


\section{Iing \\ cray expers}

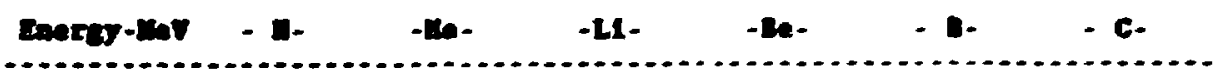

$9.800 e-04 \quad 1.101 e-12 \quad 1.104 e-11 \quad 3.694 e-11 \quad 9.085 e-11 \quad 2.030 e-10 \quad 3.742 e-10$

$9.900 e-04 \quad 1.143 e-12 \quad 1.070 e-11 \quad 3.457 e-11 \quad 9.464 e-11 \quad 1.947 e-10 \quad 3.627 e-10$

$1.000 e-03 \quad 1.120 e-12 \quad 1.048 e-11 \quad 3.312 e-11 \quad 9.207 e-11 \quad 1.896 e-10 \quad 3.556 e-10$

1.050e-03 $1.083 e-12$ 1.014a-11 $3.179 a-11 \quad 3.908 a-11 \quad 1.838--10 \quad 3.460 e-10$

1.140e-03 $1.026 e-12 \quad 9.636 e-12 \quad 3.046 e-11 \quad 0.533 e-11 \quad 1.764 e-10 \quad 3.323 e-10$

$1.3600-03 \quad 8.632 e-13 \quad 0.103 e-12 \quad 2.628 e-11 \quad 7.359 e-11 \quad 1.531 e-10 \quad 2.890 e-10$

$1.560,-03 \quad 5.224 e-13 \quad 4.937 e-12 \quad 1.745 e-11 \quad 4.093 e-11 \quad 1.035 e-10 \quad 1.968 e-10$

$1.700 e-03 \quad 3.063 e-13 \quad 3.665 e-1.21 .373 e-11 \quad 3.840 e-11 \quad 8.244 e-11 \quad 1.575 e-10$

$1.040 e-03 \quad 3.322 e-13 \quad 3.150 e-12 \quad 1.202 e-11 \quad 3.366 e-11 \quad 7.264 e-11 \quad 1.391 e-10$

1.910e-03 2.830e-13 2.682e-12 $1.046 e-11 \quad 2.931 e-11 \quad 6.361 e-11 \quad 1.222 e-10$

2.140e-03 2.215e-13 2.096e-12 8.485e-12 $2.382 e-11 \quad 5.219 e-11 \quad 1.007 e-10$

2.240e-03 2.009e-13 $1.899 e-12 \quad 7.788 e-12 \quad 2.188 e-11 \quad 4.812 e-11 \quad 9.300 e-11$

2.410e-03 $1.882 e-13 \quad 1.776 e-12 \quad 7.324 e-12 \quad 2.059 e-11 \quad 4.538 e-11 \quad 8.780 e-11$

2.470e-03 $1.753 e-13 \quad 1.652 e-12 \quad 6.853 e-12 \quad 1.929 e-11 \quad 4.258 e-11 \quad 8.249 e-11$

2.630e-03 $1.607 e-13 \quad 1.511 e-12$ 6.317e-12 $1.780 e-11 \quad 3.939 e-11 \quad 7.643 e-11$

2.750e-03 $1.396 e-13 \quad 1.308 e-12 \quad 5.540 e-12 \quad 1.564 e-11 \quad 3.475 e-11 \quad 6.761 e-11$

2.820e-03 1.236e-13 $1.153 e-12 \quad 4.947 e-12 \quad 1.398 e-11 \quad 3.121 e-11 \quad 6.085 e-11$

$2.960 e-03 \quad 1.039 e-13 \quad 9.645 e-13 \quad 4.222 e-12 \quad 1.196 e-11 \quad 2.687 e-11 \quad 5.258 e-11$

$\begin{array}{lllllll}3.180 e-03 & 8.070 e-14 & 7.405 e-13 & 3.352 e-12 & 9.540 e-12 & 2.165 e-11 & 4.263 e-11\end{array}$

3.550e-03 7.118e-14 $6.461 e-13 \quad 2.953 e-12 \quad 8.423 e-12 \quad 1.919 e-11 \quad 3.790 e-11$

3.610e-03 6.319e-14 $5.673 e-13 \quad 2.616 e-12 \quad 7.476 e-12 \quad 1.710 e-11 \quad 3.386 e-11$

3.700e-03 5.997e-14 5.356e-13 $2.480 e-12 \quad 7.095 e-12 \quad 1.626 e-11 \quad 3.223 e-11$

3.830e-03 5.491e-14 4.860e-13 $2.267 e-12 \quad 6.496 e-12 \quad 1.493 e-11 \quad 2.966 e-11$

4.030e-03 4.666e-14 4.052e-13 1.919e-12 5.518e-12 $1.277 e-11 \quad 2.547 e-11$

4.150e-03 4.185e-14 $3.575 e-13 \quad 1.711 e-12 \quad 4.933 e-12 \quad 1.146 e-11 \quad 2.294 e-11$

$4.300 e-03 \quad 4.027 e-14 \quad 3.409 e-13 \quad 1.635 e-12 \quad 4.718 e-12 \quad 1.098 e-11 \quad 2.199 e-11$

4.500e-03 3.793e-14 $3.167 e-13 \quad 1.524 e-12 \quad 4.404 e-12 \quad 1.027 e-11 \quad 2.060 e-11$

4.810e-03 3.393e-14 2.758e-13 $1.336 e-12 \quad 3.873 e-12 \quad 9.066 e-12 \quad 1.824 e-11$

$\begin{array}{lllllll}5.160 e-03 & 2.836 e-14 & 2.191 e-13 & 1.074 e-12 & 3.131 e-12 & 7.384 e-12 & 1.494 e-11\end{array}$

5.470e-03 2.579e-14 $1.909 e-13 \quad 9.410 e-13 \quad 2.752 e-12 \quad 6.515 e-12 \quad 1.322 e-11$

$\begin{array}{llllllll}5.550 e-03 & 2.454 e-14 & 1.768 e-13 & 8.733 e-13 & 2.558 e-12 & 6.069 e-12 & 1.234 e-11\end{array}$

$5.930 e-03 \quad 2.281 e-14 \quad 1.578 e-13 \quad 7.821 e-13 \quad 2.296 e-12 \quad 5.467 e-12 \quad 1.114 e-11$

$\begin{array}{lllllll}5.980 e-03 & 2.105 e-14 & 1.386 e-13 & 6.898 e-13 & 2.032 e-12 & 4.856 e-12 & 9.932 e-12\end{array}$

$\begin{array}{lllllll}6.250 e-03 & 2.054 e-14 & 1.320 e-13 & 6.574 e-13 & 1.939 e-12 & 4.641 e-12 & 9.502 e-12\end{array}$

$6.540 e-03 \quad 2.019 e-14 \quad 1.255 e-13 \quad 6.245 e-13 \quad 1.843 e-12 \quad 4.418 e-12 \quad 9.057 e-12$

$6.750 e-03 \quad 1.979 e-14 \quad 1.188 e-13 \quad 5.909 e-13 \quad 1.746 e-12 \quad 4.190 e-12 \quad 8.600 e-12$

$6.980 e-03 \quad 1.935 e-14 \quad 1.122 e-13 \quad 5.576 e-13 \quad 1.649 e-12 \quad 3.964 e-12 \quad 8.145 e-12$

$\begin{array}{lllllll}7.110 e-03 & 1.895 e-14 & 1.063 e-13 & 5.279 e-13 & 1.563 e-12 & 3.762 e-12 & 7.740 e-12\end{array}$

$7.250 e-03 \quad 1.861 e-14 \quad 1.016 e-13 \quad 5.041 e-13 \quad 1.494 e-12 \quad 3.600 e-12 \quad 7.413 e-12$

$7.710 e-03 \quad 1.775 e-14 \quad 9.006 e-14 \quad 4.461 e-13 \quad 1.325 e-12 \quad 3.204 e-12 \quad 6.617 e-12$

$7.940 e-03 \quad 1.663 e-14 \quad 7.546 e-14 \quad 3.726 e-13 \quad 1.112 e-12 \quad 2.702 e-12 \quad 5.608 e-12$

$\begin{array}{llllllll}8.180 e-03 & 1.604 e-14 & 6.716 e-14 & 3.306 e-13 & 9.894 e-13 & 2.414 e-12 & 5.028 e-12\end{array}$

8.380e-03 $1.605 e-14 \quad 6.479 e-14 \quad 3.179 e-13 \quad 9.520 e-13 \quad 2.325 e-12 \quad 4.846 e-12$

8.600e-03 $1.607 e-14 \quad 6.261 e-14 \quad 3.061 e-13 \quad 9.172 e-13 \quad 2.2416-12 \quad 4.676 e-12$

8.980e-03 1.607e-14 5.915e-14 2.875e-13 8.621e-13 $2.110 e-12 \quad 4.407 e-12$ 


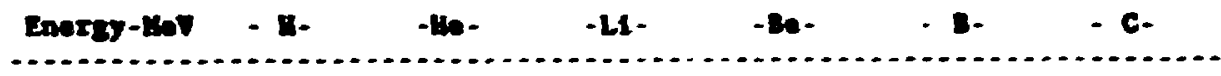

$9.200 e-03 \quad 1.603 e-14 \quad 5.533 e-14 \quad 2.671 e-13 \quad 0.017 e-13 \quad 1.965 e-12 \quad 4.111 e-12$ $9.400 e-03 \quad 1.598--14 \quad 5.243 e-14 \quad 2.516 e-13 \quad i .556 e-13 \quad 1.854 e-12 \quad 3.086 e-12$ $9.560 e-03 \quad 1.592 e-14 \quad 4.978 e-14 \quad 2.375 e-13 \quad 7.139 e-13 \quad 1.754 e-12 \quad 3.681 e-12$ 9.800e-03 $1.583 e-14 \quad 4.667 e-14 \quad 2.210 e-13 \quad 6.649 e-13 \quad 1.637 e-12 \quad 3.440 e-12$ 1.000e-02 $1.571 e-14 \quad 4.306 e-14 \quad 2.018 e-13 \quad 6.080 e-13 \quad 1.500 e-12 \quad 3.161 e-12$ $1.040 e-02 \quad 1.735 e-14 \quad 4.175 e-14 \quad 1.913 e-13 \quad 5.755 e-13 \quad 1.421 e-12 \quad 2.999 e-12$ 1.080e-02 2.074e-14 $4.242 e-14 \quad 1.877 e-13 \quad 5.623 e-13 \quad 1.387 e-12 \quad 2.928 e-12$ $1.110 e-02 \quad 2.370 e-14 \quad 4.288 e-14 \quad 1.839 e-13 \quad 5.486 e-13 \quad 1.352 e-12 \quad 2.854 e-12$ 1.190e-02 2.833e-14 4.334e-14 $1.764 e-13 \quad 5.222 e-13 \quad 1.286 e-12 \quad 2.714 e-12$ $1.270 e-02 \quad 3.503 e-14 \quad 4.352 e-14 \quad 1.626 e-13 \quad 4.752 e-13 \quad 1.167 e-12 \quad 2.464 e-12$ $1.300 e-02 \quad 3.961 e-14 \quad 4.331 e-14 \quad 1.511 e-13 \quad 4.368 e-13 \quad 1.070 e-12 \quad 2.261 e-12$

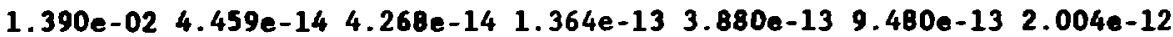
1.470e-02 5.159e-14 4.125e-14 $1.124 e-13 \quad 3.092 e-13 \quad 7.506 e-13 \quad 1.589 e-12$ $\begin{array}{lllllll}1.520 e-02 & 5.694 e-14 & 3.994 e-14 & 9.271 e-14 & 2.449 e-13 & 5.894 e-13 & 1.250 e-12\end{array}$ 1.570e-02 6.123e-14 $4.112 e-14 \quad 8.931 e-14 \quad 2.308 e-13 \quad 5.521 e-13 \quad 1.170 e-12$ $1.640 \mathrm{e}-02 \quad 6.642 \mathrm{e}-14 \quad 4.314 \mathrm{e}-14 \quad 8.850 \mathrm{e}-14 \quad 2.237 \mathrm{e}-13 \quad 5.312 \mathrm{e}-13 \quad 1.123 e-12$ $1.700 e-02 \quad 7.202 e-14 \quad 4.526 e-14 \quad 8.719 e-14 \quad 2.147 e-13 \quad 5.050 e-13 \quad 1.065 e-12$ $1.720 e-02 \quad 7.547 e-14 \quad 4.652 e-14 \quad 8.616 e-14 \quad 2.084 e-13 \quad 4.871 e-13 \quad 1.025 e-12$ $1.810 e-02 \quad 8.019 e-14 \quad 4.820 e-14 \quad 8.441 e-14 \quad 1.987 e-13 \quad 4.597 e-13 \quad 9.648 e-13$ $1.900 e-028.789 e-14 \quad 5.085 e-14 \quad 8.087 e-14 \quad 1.807 e-13 \quad 4.094 e-13 \quad 8.539 e-13$ $1.970 e-029.470 e-14 \quad 5.308 e-14 \quad 7.699 e-14 \quad 1.623 e-13 \quad 3.587 e-13 \quad 7.425 e-13$ 2.010e-02 9.937e-14 5.456e-14 $7.403 e-14 \quad 1.488 e-13 \quad 3.215 e-13 \quad 6.606 e-13$ 2.100e-02 $1.049 e-13 \quad 5.704 e-14 \quad 7.481 e-14 \quad 1.462 e-13 \quad 3.114 e-13 \quad 6.364 e-13$ 2.170e-02 $1.117 e-13 \quad 6.027 e-14 \quad 7.686 e-14 \quad 1.464 e-13 \quad 3.075 e-13 \quad 6.247 e-13$ $\begin{array}{lllllll}2.230 e-02 & 1.172 e-13 & 6.287 e-14 & 7.840 e-14 & 1.462 e-13 & 3.034 e-13 & 6.130 e-13\end{array}$ $2.310 e-02 \quad 1.231 e-13 \quad 6.565 e-14 \quad 7.994 e-14 \quad 1.457 e-13 \quad 2.980 e-13 \quad 5.984 e-13$ $2.550 e-02 \quad 1.364 e-13 \quad 7.189 e-14 \quad 8.299 e-14 \quad 1.428 e-13 \quad 2.816 e-13 \quad 5.558 e-13$ $2.920 e-02 \quad 1.616 e-13 \quad 8.343 e-14 \quad 8.710 e-14 \quad 1.318 e-13 \quad 2.361 e-13 \quad 4.437 e-13$ 3.320e-02 $1.928 e-13 \quad 9.791 e-14 \quad 9.298 e-14 \quad 1.205 e-13 \quad 1.853 e-13 \quad 3.163 e-13$ $3.750 e-022.255 e-13 \quad 1.141 e-13 \quad 1.050 e-13 \quad 1.277 e-13 \quad 1.807 e-13 \quad 2.887 e-13$ 4.200e-02 2.594e-13 $1.308 e-13 \quad 1.172 e-13 \quad 1.338 e-13 \quad 1.725 e-13 \quad 2.518 e-13$

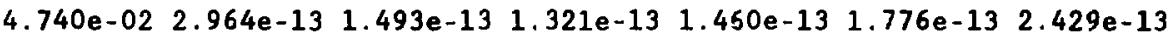
$4.850 e-02 \quad 3.202 e-13 \quad 1.613 e-13 \quad 1.418 e-13 \quad 1.541 e-13 \quad 1.817 e-13 \quad 2.389 e-13$ $\begin{array}{lllllll}5.020 e-02 & 3.303 e-13 & 1.663 e-13 & 1.459 e-13 & 1.574 e-13 & 1.829 e-13 & 2.360 e-13\end{array}$

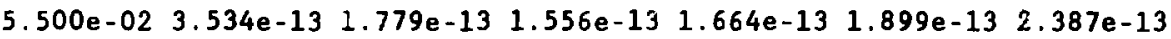
$6.100 e-02 \quad 3.909 e-13 \quad 1.968 e-13 \quad 1.716 e-13 \quad 1.813 e-13 \quad 2.021 e-13 \quad 2.451 e-13$ $6.750 e-02 \quad 4.332 e-13 \quad 2.182 e-13 \quad 1.897 e-13 \quad 1.990 e-13 \quad 2.182 e-13 \quad 2.578 e-13$

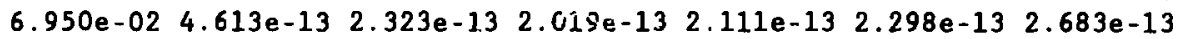
$\begin{array}{lllllll}7.350 e-02 & 4.806 e-13 & 2.421 e-13 & 2.102 e-13 & 2.194 e-13 & 2.375 e-13 & 2.750 e-13\end{array}$ $\begin{array}{lllllll}7.840 e-02 & 5.086 e-13 & 2.562 e-13 & 2.223 e-13 & 2.312 e-13 & 2.484 e-13 & 2.838 e-13\end{array}$ 8.070e-02 5.308,-13 2.674e-13 $2.318 \mathrm{e}-13 \quad 2.405 \mathrm{e}-13 \quad 2.568 \mathrm{e}-13 \quad 2.902 \mathrm{e}-13$ 8.550e-02 5.526e-13 $2.784 e-13 \quad 2.413 e-13 \quad 2.501 e-13 \quad 2.663 e-13 \quad 2.995 e-13$ $\begin{array}{lllllll}8.800 e-0 z & 5.747 e-13 & 2.896 e-13 & 2.509 e-13 & 2.599 e-13 & 2.762 e-13 & 3.095 e-13\end{array}$ $\begin{array}{lllllll}9.050 e-02 & 5.896 e-13 & 2.971 e-13 & 2.574 e-13 & 2.664 e-13 & 2.827 e-13 & 3.160 e-13\end{array}$

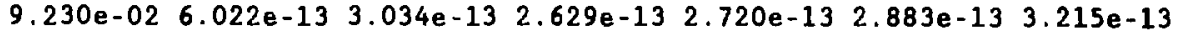


rin:

Geay erinz

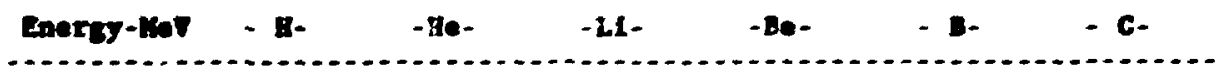

$9.600-02 \quad 6.100 e-13 \quad 3.114 e-13 \quad 2.698 e-13 \quad 2.789 e-13 \quad 2.952 \bullet-13 \quad 3.292 e-13$

$\begin{array}{llllllll}1.000 e-01 & 6.399 e-13 & 3.225 e-13 & 2.793 e-13 & 2.885 e-13 & 3.047 e-13 & 3.373 e-13\end{array}$

$\begin{array}{lllllll}1.050 e-01 & 6.937 \bullet-13 & 3.495 e-13 & 3.027 e-13 & 3.124 e-13 & 3.292 e-13 & 3.632 e-13\end{array}$

1.100e-01 $7.778 e-13 \quad 3.919 e-13 \quad 3.392 e-13 \quad 3.498 e-13 \quad 3.680 e-13 \quad 4.047 e-13$

$1.150 e-01 \quad 8.601 e-13 \quad 4.333 e-13 \quad 3.750 e-13 \quad 3.865 e-13 \quad 4.060 e-13 \quad 4.455 e-13$

$1.210 e-01 \quad 9.485 e-13 \quad 4.777 e-13 \quad 4.134 e-13 \quad 4.258 e-13 \quad 4.468 e-13 \quad 4.891 e-13$

$1.350 e-01 \quad 1.103 e-12 \quad 5.555 e-13 \quad 4.806 e-13 \quad 4.946 e-13 \quad 5.181 e-13 \quad 5.652 e-13$

$1.490 e-01 \quad 1.308 e-12 \quad 6.587 e-13 \quad 5.698 e-13 \quad 5.859 e-13 \quad 6.124 e-13 \quad 6.655 e-13$

$\therefore .640 e-01 \quad 1.510 e-12 \quad 7.605 e-13 \quad 6.579 e-13$ c i61e-13 $7.059 e-13 \quad 7.653 e-13$

i. $.800 e-01 \quad 1.719 e-128.658 e-13 \quad 7.489 e-13 \quad 7.695 e-13 \quad 8.030 e-13 \quad 8.698 e-13$

$\begin{array}{lllllll}1.980 e-01 & 1.933 e-12 & 9.735 e-13 & 8.420 e-13 & 8.651 e-13 & 9.024 e-13 & 9.766 e-13\end{array}$

2.180e-01 2.163e-12 $1.089 e-12 \quad 9.420 e-13 \quad 9.677 e-13 \quad 1.009 e-12 \quad 1.091 e-12$

2.390e-01 2.405e-12 $1.211 e-12 \quad 1.048 e-12 \quad 1.076 e-12 \quad 1.122 e-12 \quad 1.213 e-12$

$\begin{array}{lllllll}2.630 e-01 & 2.652 e-12 & 1.336 e-12 & 1.155 e-12 & 1.187 e-12 & 1.237 e-12 & 1.338 e-12\end{array}$

2.900e-01 2.908e-12 $1.465 e-12 \quad 1.267 e-12 \quad 1.301 e-12 \quad 1.356 e-12 \quad 1.466 e-12$

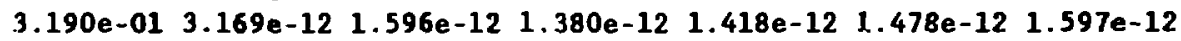

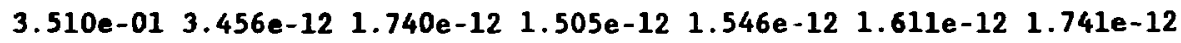

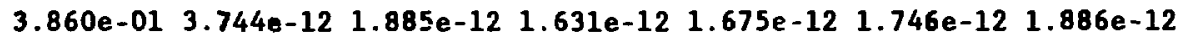

$\begin{array}{lllllll}4.240 e-01 & 4.031 e-12 & 2.030 e-12 & 1.756 e-12 & 1.804 e-12 & 1.880 e-12 & 2.031 e-12\end{array}$

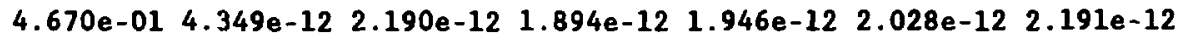

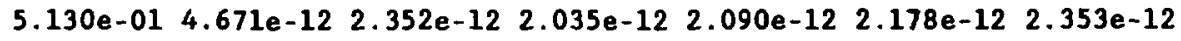

$\begin{array}{lllllll}5.650 e-01 & 5.153 e-12 & 2.595 e-12 & 2.245 e-12 & 2.306 e-12 & 2.403 e-12 & 2.596 e-12\end{array}$

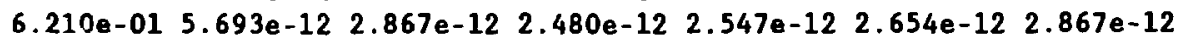

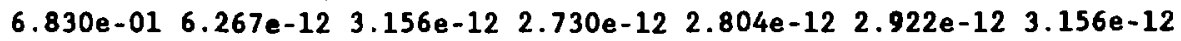

$\begin{array}{lllllll}7.510 e-01 & 6.845 e-12 & 3.447 e-12 & 2.982 e-12 & 3.062 e-12 & 3.191 e-12 & 3.447 e-12\end{array}$

8.260e-01 7.405e-12 $3.729 e-12 \quad 3.226 e-12 \quad 3.313 e-12 \quad 3.453 e-12 \quad 3.729 e-12$

9.090e-01 $8.017 e-12 \quad 4.037 e-12 \quad 3.492 e-12 \quad 3.587 e-12 \quad 3.738 e-12 \quad 4.037 e-12$

$\begin{array}{lllllll}1.000 e+00 & 8.626 e-12 & 4.344 e-12 & 3.757 e-12 & 3.859 e-12 & 4.021 e-12 & 4.343 e-12\end{array}$

$\begin{array}{lllllll}1.120 e+00 & 9.443 e-12 & 4.756 e-12 & 4.114 e-12 & 4.224 e-12 & 4.402 e-12 & 4.755 e-12\end{array}$

$\begin{array}{lllllll}1.270 e+00 & 1.052 e-11 & 5.300 e-12 & 4.585 e-12 & 4.708 e-12 & 4.907 e-12 & 5.300 e-12\end{array}$

$\begin{array}{lllllll}1.420 e+00 & 1.153 e-11 & 5.806 e-12 & 5.023 e-12 & 5.159 e-12 & 5.376 e-12 & 5.807 e-12\end{array}$

$\begin{array}{lllllll}1.600 e+00 & 1.245 e-11 & 6.269 e-12 & 5.424 e-12 & 5.571 e-12 & 5.807 e-12 & 6.272 e-12\end{array}$

$\begin{array}{lllllll}1.800 e+00 & 1.354 e-11 & 6.825 e-12 & 5.907 e-12 & 6.068 e-12 & 6.327 e-12 & 6.837 e-12\end{array}$

$\begin{array}{lllllll}2.030 e+00 & 1.458 e-11 & 7.348 e-12 & 6.362 e-12 & 6.539 e-12 & 6.821 e-12 & 7.373 e-12\end{array}$

$2.280 e+00 \quad 1.586 e-11 \quad 7.999 e-12 \quad 6.930 e-12 \quad 7.128 e-12 \quad 7.439 e-12 \quad 8.047 e-12$

$2.570 e+00 \quad 1.729 e-11 \quad 8.728 e-12 \quad 7.569 e-12 \quad 7.791 e-12 \quad 8.139 e-12 \quad 8.812 e-12$

$2.890 e+001.855 e-11 \quad 9.377 e-12 \quad 8.142 e-12 \quad 8.391 e-12 \quad 8.777 e-12 \quad 9.514 e-12$

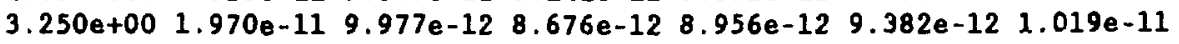

$3.650 e+002.114 e-11 \quad 1.072 e-11 \quad 9.341 e-12 \quad 9.660 e-12 \quad 1.014 e-11 \quad 1.103 e-11$ $4.110 e+002.239 e-11 \quad 1.139 e \quad 11 \quad 9.944 e-12 \quad 1.031 e-11 \quad 1.084 e-11 \quad 1.182 e-11$ $\begin{array}{lllllll}4.620 e+00 & 2.390 e-11 & 1.219 e-11 & 1.067 e-11 & 1.109 e-11 & 1.170 e-11 & 1.279 e-11\end{array}$ $\begin{array}{lllllll}5.200 e+00 & 2.539 e-11 & 1.299 e-11 & 1.141 e-11 & 1.190 e-11 & 1.259 e-11 & 1.380 e-11\end{array}$ $5.850 e+002.699 e-11 \quad 1.386 e-11 \quad 1.221 e-11 \quad 1.278 e-11 \quad 1.356 e-11 \quad 1.491 e-11$ $6.580 e+002.866 e-11 \quad 1.478 e-11 \quad 1.308 e-11 \quad 1.373 e-11 \quad 1.463 e-11 \quad 1.614 e-11$ $7.410 e+003.066 e-11 \quad 1.588 e-11 \quad 1.412 e-11 \quad 1.489 e-11 \quad 1.592 e-11 \quad 1.763 e-11$ 


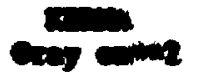

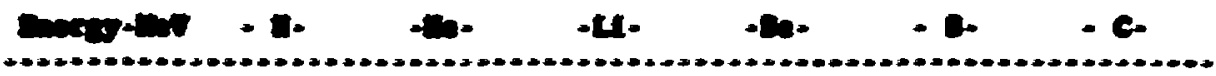

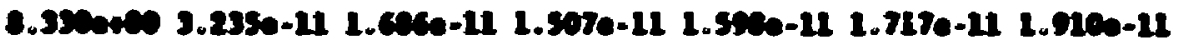

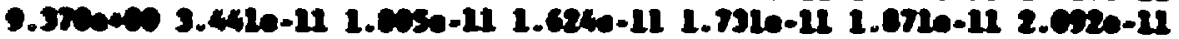

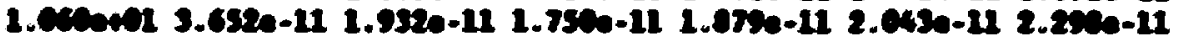

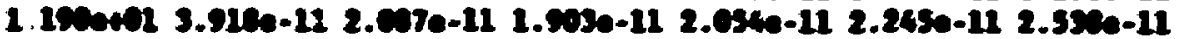
1.34061 4.1750-11 2.2410-11 2.0500-11 $2.2360-11$ 2.4570-11 2.7910-11 2.cosevel 4.7750-11 2.629e-11 $2.4670-112.7310-11$ 3.0320-11 3.3170-11 3.c000101 6.076e-11 3.403e-11 3.377e-11 3.0410-11 4.350e-11 5.150e-11 4.0000+01 7.450e-11 4.4620-11 4.454e-11 3.1000-11 6.027e-11 7.1830-11 5.6000+01 8.915e-11 5.4730-11 5.576e-11 6.5070-11 7.7580-11 9.3230-11 7.500e+01 1.150e-10 7.323e-11 7.641e-11 $9.1710-11$ 1.032e-10 $1.3240-10$ 1.000e4J2 1.532e-10 1.009e-10 1.075e-10 1.307e-10 1.570a-10 1.917e-10 


\section{$1.0000-04$}

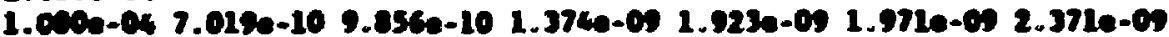

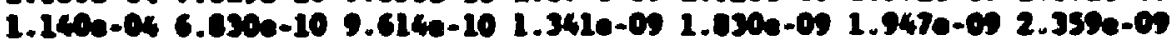

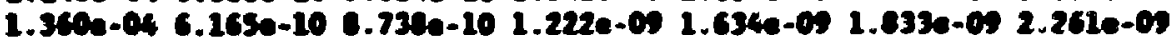

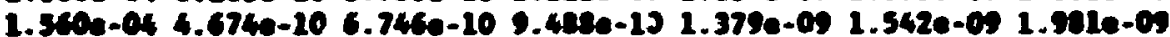

1.700e-e4 4.015e-10 5.e47e-10 8.242e-10 1.242e-0s 1.396e-0s 1.825e-0s

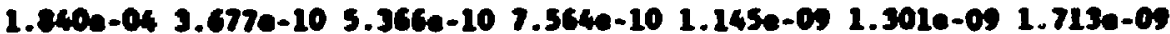

1.910e-04 3.357e-10 4.90se-10 6.918e-10 1.051e-0s 1.210e-0s 1.602e-0s

2.140e-04 2.9430-10 4.316e-10 $6.092 e-10 \quad 9.215 e-10$ 1.05ee-09 1.454e-0s

2.240e-04 2.793e-10 4.09te-10 $5.774 e-10$ e.466e-10 $1.042 e-05$ 1.396e-0s

2.410e-04 2.601e-10 3.934e-10 $5.542 e-107.811 e-10$ 1.004e-0s $1.345 e-0 s$

2.470e-04 $2.563 e-10 \quad 3.761 e-10 \quad 5.297 e-10 \quad 7.160 e-10 \quad 9.641 e-10 \quad 1.296 e-09$

2.630e-04 2.425e-10 $3.558 e-10 \quad 5.010 e-10 \quad 6.672 e-10 \quad 9.165 e-10 \quad 1.234 e-09$

2.750e-04 $2.220 e-10 \quad 3.257 e-10 \quad 4.585 e-10 \quad 6.293 e-10 \quad 8.456 e-10 \quad 1.142 e-09$

2.820e-04 2.061e-10 $3.024 e-10 \quad 4.255 e-10 \quad 6.001 e-10 \quad 7.903 e-10 \quad 1.071 e-09$

$2.960 e-04 \quad 1.865 e-10 \quad 2.736 e-10 \quad 3.848 e-10 \quad 5.633 e-10 \quad 7.218 e-10 \quad 9.812 e-10$

$3.180 e-041.626 e-10 \quad 2.384 e-10 \quad 3.349 e-10 \quad 5.145 e-10 \quad 6.374 e-10 \quad 8.709 e-10$

3.550e-04 1.500e-10 $2.198 e-10 \quad 3.082 e-10 \quad 4.738 e-10 \quad 5.900 e-10 \quad 8.077 e-10$

3.610e-04 1.387e-10 2.032e-10 2.844e-10 4.367e-10 5.472e-10 7.503e-10

3.700e-04 1.341e-10 $1.964 e-10 \quad 2.747 e-10 \quad 4.214 e-10 \quad 5.296 e-10 \quad 7.266 e-10$

3.830e-04 1.267e-10 1.855e-10 2.591e-10 3.972e-10 5.014e-10 $6.889 e-10$

4.030e-04 2.411e-10 $1.676 e-10 \quad 2.334 e-10 \quad 3.572 e-10 \quad 4.549 e-10 \quad 6.264 e-10$

4.150e-04 1.901e-09 1.566e-10 2.176e-10 3.325e-10 4.258e-10 5.871e-10

4.300e-04 1.863e-09 1.521e-10 2.112e-10 3.225e-10 4.135e-10 5.705e-10

4.500e-04 1.803e-09 $1.455 e-10 \quad 2.016 e-10 \quad 3.074 e-10 \quad 3.950 e-10 \quad 5.454 e-10$

4.810e-04 1.697e-09 1.338e-10 1.849e-10 2.814e-10 3.629e-10 5.018e-10

5.160e-04 1.539e-09 $1.168 e-10 \quad 1.610 e-10 \quad 2.443 e-10 \quad 3.169 e-10 \quad 4.394 e-10$

5.470e-04 1.434e-09 B.388e-10 1.478e-10 2.240e-10 2.916e-10 4.051e-10

5.550e-04 1.372e-09 1.608e-09 $1.407 e-10 \quad 2.132 e-10 \quad 2.779 e-10 \quad 3.865 e-10$

5.930e-04 $1.286 e-091.579 e-09 \quad 1.308 e-10 \quad 1.983 e-10 \quad 2.591 e-10 \quad 3.609 e-10$

5.980e-04 $1.198 e-09 \quad 1.546 e-09 \quad 1.208 e-10 \quad 1.830 e-10 \quad 2.398 e-10 \quad 3.346 e-10$

6.250e-04 1.156e-09 $1.510 e-09 \quad 1.161 e-10 \quad 1.760 e-10 \quad 2.307 e-10 \quad 3.221 e-10$

6.540e-04 1.095e-09 $1.434 e-09 \quad 1.093 e-10 \quad 1.662 e-10 \quad 2.279 e-10 \quad 3.043 e-10$

6.750e-04 1.033e-09 $1.356 e-09 \quad 1.025 e-10 \quad 1.562 e-10 \quad 2.047 e-10 \quad 2.860 e-10$

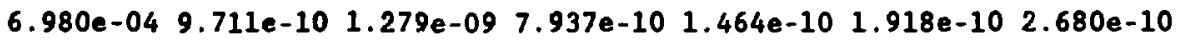

7.110e-04 9.245e-10 1.221e-09 $1.402 e-09 \quad 1.389 e-10 \quad 1.821 e-10 \quad 2.546 e-10$

7.250e-04 9.046e-10 $1.198 \mathrm{e}-09 \quad 1.386 \mathrm{e}-09 \quad 1.358 \mathrm{e}-10 \quad 1.783 \mathrm{e}-10 \quad 2.494 \mathrm{e}-10$

$7.710 e-04 \quad 8.552 e-101.139 e-09 \quad 1.341 e-09 \quad 1.279 e-10 \quad 1.686 e-10 \quad 2.365 e-10$

7.940e-04 7.911e-10 1.061e-09 1.282e-09 $1.178 \mathrm{e}-10 \quad 1.561 \mathrm{e}-10 \quad 2.197 \mathrm{e}-10$

8.180e-04 7.493e-10 $1.010 e-09 \quad 1.238 e-09 \quad 1.110 e-10 \quad 1.479 e-10 \quad 2.085 e-10$

$8.380 e-047.238 e-10 \quad 9.765 e-10 \quad 1.199 e-09 \quad 1.066 e-10 \quad 1.426 e-10 \quad 2.009 e-10$

8.600e-04 6.979e-10 $9.422 e-10 \quad 1.159 e-09 \quad 1.021 e-10 \quad 1.372 e-10 \quad 1.932 e-10$

$8.980 e-04 \quad 6.570 e-10 \quad 8.877 \mathrm{e}-10 \quad 1.095 \mathrm{e}-09 \quad 1.123 \mathrm{e}-09 \quad 1.288 \mathrm{e}-10 \quad 1.810 \mathrm{e}-10$

$9.200 e-04 \quad 6.200 e-10 \quad 8.384 e-10 \quad 1.036 e-09 \quad 1.373 e-09 \quad 1.221 e-10 \quad 1.710 e-10$

$9.400 e-046.046 e-108.178 e-101.011 e-09 \quad 1.342 e-091.211 e-101.686 e-10$

$9.560 e-045.904 e-10 \quad 7.988 e-10 \quad 9.873 e-10 \quad 1.313 e-09 \quad 1.202 e-10 \quad 1.664 e-10$ 


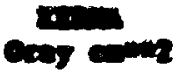

\begin{tabular}{|c|}
\hline 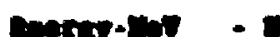 \\
\hline
\end{tabular}

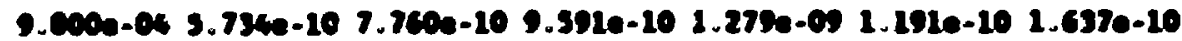

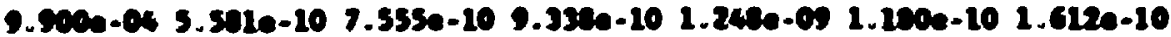

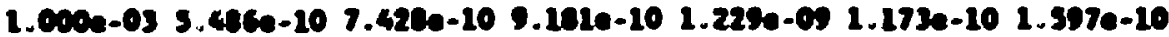

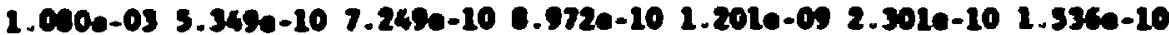
$1.1400-035.1450-10 \quad 0.4060-10 \quad 0.6720-101.1610-091.213 e-091.425 e-10$

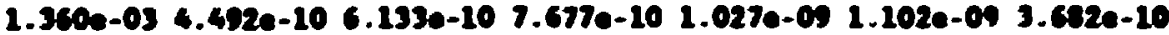

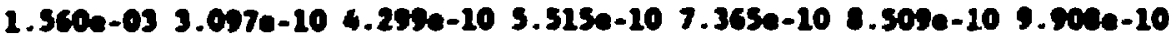
$1.700-032.4040-103.305 e-104.5640-10 \quad 6.092 e-107.305 e-10 \quad 0.0710-10$ $1.840 e-03 \quad 2.214 e-103.121 e-104.09 \%-10 \quad 5.459 e-10 \quad 5.607 e-10 \quad 0.072 e-10$ 1.910e-03 $1.952 e-102.765 e-103.646 e-10 \quad 4.970 e-10 \quad 5.933 e-10 \quad 7.318 e-10$ 2.140e-03 1.619e-10 2.312e-10 $3.092 e-10 \quad 4.119 e-10 \quad 5.111 \bullet-10 \quad 6.344 e-10$ 2.240e-03 1.500e-10 2.146e-10 2.875e-10 3.044e-10 4.798e-10 5.978e-10 2.410e-03 1.416e-10 2.035e-10 2.729e-10 3.650e-10 $4.570 e-10 \quad 5.707 e-10$ 2.470e-03 $1.3340-101.918 e-10 \quad 2.578 e-10 \quad 3.451 e-10 \quad 4.334 e-10 \quad 5.425 e-10$ 2.630e-03 1.239e-10 $1.795 e-10 \quad 2.405 e-10 \quad 3.221 e-10 \quad 4.061 e-10 \quad 5.096 e-10$ 2.750e-03 1.099e-10 $1.590 e-10 \quad 2.153 e-10 \quad 2.885 e-10 \quad 3.661 e-10 \quad 4.614 e-10$ $2.820 e-03 \quad 9.921 e-11 \quad 1.440 e-10 \quad 1.958 e-10 \quad 2.627 e-10 \quad 3.352 e-10 \quad 4.242 e-10$ $2.960 e-03 \quad 0.611 e-11 \quad 1.257 e-10 \quad 1.720 e-10 \quad 2.310 e-10 \quad 2.972 e-10 \quad 3.784 e-10$ $\begin{array}{lllllll}3.180 e-03 & 7.032 e-11 & 1.035 e-10 & 1.431 e-10 & 1.925 e-10 & 2.510 e-10 & 3.224 e-10\end{array}$ 3.550e-03 6.269e-11 $9.261 e-11 \quad 1.285 s-10 \quad 1.731 e-10 \quad 2.267 e-10 \quad 2.923 e-10$ 3.610e-03 5.616e-11 $8.324 e-11 \quad 1.159 e-10 \quad 1.564 e-10 \quad 2.056 e-10 \quad 2.660 e-10$ 3.700e-03 5.352e-11 $7.345 e-11 \quad 1.108 e-10 \quad 1.496 e-10 \quad 1.971 e-10 \quad 2.553 e-10$ $3.830 e-03 \quad 4.937 e-11 \quad 7.347 e-11 \quad 1.027 e-10 \quad 1.388 e-10 \quad 1.835 e-10 \quad 2.383 e-10$ 4.030e-03 4.257e-11 $6.369 e-11 \quad 8.951 e-11 \quad 1.212 e-10 \quad 1.613 e-10 \quad 2.105 e-10$ 4.150e-03 3.846e-11 5.776e-11 8.147e-11 $1.105 e-10 \quad 1.476 e-10 \quad 1.934 e-10$ 4.300e-03 3.691e-11 5.549e-11 7.836e-11 $1.064 e-10 \quad 1.42:--10 \quad 1.865 e-10$ 4.500e-03 3.463e-11 5.216e-11 7.377e-11 $1.002 e-10 \quad 1.343 e-10 \quad 1.764 e-10$ 4.810e-03 3.076e-11 4.648e-11 $6.595 e-11 \quad 8.975 e-11 \quad 1.206 e-10 \quad 1.589 e-10$ 5.160e-03 2.533e-11 3.851e-11 $5.494 e-11 \quad 7.500 e-11 \quad 1.014 e-10 \quad 1.343 e-10$ 5.470e-03 2.249e-11 3.431e-11 4.910e-11 6.717e-11 9.110e-11 $1.210 e-10$ 5.550e-03 2.102e-11 3.213e-11 4.604e-11 6.306e-11 8.565e-11 $1.140 e-10$ 5.930e-03 $1.903 e-11 \quad 2.917 e-11 \quad 4.190 e-11 \quad 5.748 e-11 \quad 7.825 e-11 \quad 1.043 e-10$ 5.980e-03 1.701e-11 2.617e-11 $3.769 e-11 \quad 5.182 e-11 \quad 7.073 e-11 \quad 9.458 e-11$ 6.250e-03 1.630e-11 2.510e-11 3.618e-11 4.979e-11 6.802e-11 9.104e-11 6.540e-03 1.555e-11 2.397e-11 $3.459 e-11 \quad 4.763 e-11 \quad 6.512 e-11 \quad 8.723 e-11$ 6.750e-03 1.478e-11 2.281e-11 3.295e-11 4.541e-11 6.212e-11 8.329e-11 6.980e-03 1.401e-11 2.165e-11 3.131e-11 4.319e-11 5.912e-11 $7.935 e-11$ 7.110e-03 $1.333 e-11 \quad 2.062 e-11 \quad 2.984 e-11 \quad 4.120 e-11 \quad 5.644 e-11 \quad 7.582 e-11$ $7.250 e-03 \quad 1.278 e-11 \quad 1.979 e-11 \quad 2.866 e-11 \quad 3.960 e-11 \quad 5.428 e-11 \quad 7.297 e-11$ $7.710 e-03 \quad 1.143 e-11 \quad 1.776 e-11 \quad 2.577 e-11 \quad 3.568 e-11 \quad 4.899 e-11 \quad 6.599 e-11$ 7.940e-03 9.729e-12 $1.518 \mathrm{e}-11 \quad 2.211 \mathrm{e}-11 \quad 3.071 e-11 \quad 4.227 e-11 \quad 5.713 e-11$ 8.180e-03 8.747e-12 $1.370 e-11 \quad 1.999 e-11 \quad 2.783 e-11 \quad 3.838 e-11 \quad 5.198 e-11$ 8.380e-03 8.438e-12 $1.322 e-11 \quad 1.931 e-11 \quad 2.691 e-11 \quad 3.711 e-11 \quad 5.029 e-11$ 8.600e-03 8.148e-12 1.278e-11 $1.867 e-11 \quad 2.603 e-11 \quad 3.591 e-11 \quad 4.870 e-11$ $8.980 e-03 \quad 7.688 e-12 \quad 1.207 e-11 \quad 1.765 e-11 \quad 2.464 e-11 \quad 3.400 e-11 \quad 4.616 e-11$ 


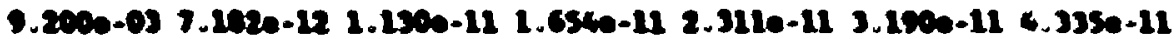

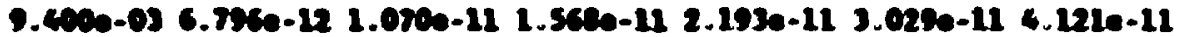
9.5xe-03 6.446-12 1.016e-11 $1.450-112.077 e-112.0030-11 \quad 3.926 e-12$ 9.000-03 6.033e-12 9.534e-12 $1.3940-11$ 1.962e-11 $2.711 e-11$ 3.696-11 1.600-02 5.355e-12 e.794e-12 $1.2930-11$ 1.816e-11 $2.512 e-11$ 3.429e-11

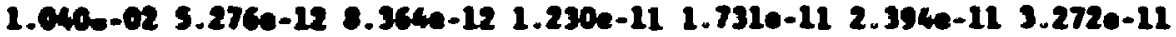
1.000-e2 5.152e-12 8.170e-12 1.202e-11 $1.632 e-12$ 2.341e-11 3.200e-11 1.110e-02 $5.023 e-12 \quad 7.965 e-12 \quad 1.173 e-11 \quad 1.651 e-112.205 e-11 \quad 3.1240-11$ $1.1500-02$ 4.776e-12 $7.546 e-12$ 1.117e-11 $1.576 e-112.177 e-112.975 e-11$ 1.270e-02 $4.342 e-12 \quad 6.9040-12 \quad 1.017 e-11$ 1.436e-11 $1.986 e-112.720 e-11$ 1.300e-02 3.94e-12 $6.349 e-12 \quad 9.359 e-12 \quad 1.323 e-11 \quad 1.8300-11 \quad 2.509 e-11$ 1.350e-02 3.539e-12 $5.645 e-12 \quad 6.330 e-12 \quad 1.160 e-11 \quad 1.6330-112.240 e-11$ 1.470e-02 2.815e-12 $4.509 e-12 \quad 6.666 e-12 \quad 9.489 e-12 \quad 1.313 e-11 \quad 1.007 e-11$ 1.520e-02 2.223e-12 3.580e-12 $5.306 e-12 \quad 7.599 e-12 \quad 1.051 e-11 \quad 1.452 e-11$ 1.570e-02 $2.001 e-12 \quad 3.355 e-12 \quad 4.975 e-12 \quad 7.136 e-12 \quad 9.869 e-12 \quad 1.364 e-11$ 1.640e-02 1.996e-12 3.219e-12 $4.773 e-12 \quad 6.850 e-12 \quad 9.471 e-12 \quad 1.310 e-11$ 1.700e-02 1.491e-12 $3.050 e-12$ 4.521e-12 $\quad 6.495 e-12 \quad 6.976 e-12 \quad 1.242 e-11$ $1.720 e-02 \quad 1.819 e-12 \quad 2.935 e-12 \quad 4.350 e-12 \quad 6.253 e-12 \quad 8.639 e-12 \quad 1.195 e-11$ 1.810e-02 $1.710 e-12 \quad 2.759 e-12 \quad 4.089 e-12 \quad 5.883 e-12 \quad 8.125 e-12 \quad 1.125 e-11$ 1.900e-02 1.511e-12 2.438e-12 $3.613 e-12 \quad 5.209 e-12 \quad 7.187 e-12 \quad 9.958 e-12$ 1.970e-02 1.311e-12 $2.117 e-12 \quad 3.135 e-12 \quad 4.532 e-12 \quad 6.245 e-12 \quad 8.663 e-12$ 2.010e-02 1.164e-12 $1.880 e-12 \quad 2.784 e-12 \quad 4.035 e-12 \quad 5.553 e-12 \quad 7.711 e-12$ 2.100e-02 1.118e-12 $1.806 e-12 \quad 2.673 e-12 \quad 3.876 e-12 \quad 5.331 e-12 \quad 7.405 e-12$ 2.170e-02 $1.095 e-12 \quad 1.766 e-12 \quad 2.611 e-12 \quad 3.787 e-12 \quad 5.206 e-12 \quad 7.231 e-12$ $\begin{array}{lllllll}2.230 e-02 & 1.072 e-12 & 1.727 e-12 & 2.552 e-12 & 3.701 e-12 & 5.086 e-12 & 7.063 e-12\end{array}$ $\begin{array}{lllllll}2.310 e-02 & 1.043 e-12 & 1.679--12 & 2.478 e-12 & 3.595 e-12 & 4.937 e-12 & 6.857 e-12\end{array}$ 2.550e-02 9.612e-13 $1.542 e-12 \quad 2.271 e-12 \quad 3.295 e-12 \quad 4.517 e-12 \quad 6.273 e-12$ 2.920e-02 $7.489 e-13 \quad 1.189 e-12 \quad 1.738 e-12 \quad 2.525 e-12 \quad 3.443 e-12 \quad 4.779 e-12$ 3.320e-02 $5.062 e-13 \quad 7.837 e-13 \quad 1.125 e-12 \quad 1.636 e-12 \quad 2.204 e-12 \quad 3.056 e-12$ 3.750e-02 4.421e-13 6.672e-13 $9.415 e-13 \quad 1.362 e-12 \quad 1.817 e-12 \quad 2.511 e-12$ 4.200e-02 $3.599 e-13 \quad 5.199 e-13 \quad 7.111 e-13 \quad 1.017 e-12 \quad 1.334 e-12 \quad 1.831 e-12$ 4.740e-02 3.276e-13 4.536e-13 $6.011 e-13 \quad 8.463 e-13 \quad 1.093 e-12 \quad 1.485 e-12$ 4.850e-02 3.097e-23 4.155e-13 $5.370 e-13 \quad 7.459 e-13 \quad 9.496 e-13 \quad 1.280 e-12$ 5.020e-02 $2.998 e-13 \quad 3.955 e-13 \quad 5.041 e-13 \quad 6.948 e-13 \quad 8.774 e-13 \quad 1.177 e-12$ 5.500e-02 $2.948 e-13 \quad 3.790 e-13 \quad 4.727 e-13 \quad 6.428 e-13 \quad 8.015 e-13 \quad 1.066 e-12$ 6.100e-02 2.900e-13 $3.578 e-13 \quad 4.301 e-13 \quad 5.706 e-13 \quad 6.952 e-13 \quad 9.101 e-13$ 6.750e-02 2.951e-13 $3.514 e-13 \quad 4.083 e-13 \quad 5.284 e-13 \quad 6.290 e-13 \quad 8.092 e-13$ $\begin{array}{llllllll}6.950 e-02 & 3.022 e-13 & 3.536 e-13 & 4.036 e-13 & 5.147 e-13 & 6.050 e-13 & 7.702 e-13\end{array}$ $\begin{array}{lllllll}7.350 e-02 & 3.060 e-13 & 3.531 e-13 & 3.973 e-13 & 5.007 e-13 & 5.821 e-13 & 7.343 e-13\end{array}$ $\begin{array}{lllllll}7.840 e-02 & 3.100 e-13 & 3.497 e-13 & 3.841 e-13 & 4.741 e-13 & 5.405 e-13 & 6.705 e-13\end{array}$ 8.070e-02 $3.120 e-13 \quad 3.452 e-13 \quad 3.709 e-13 \quad 4.488 e-13 \quad 5.018 e-13 \quad 6.119 e-13$ 8.550e-02 $3.197 e-13 \quad 3.505 e-13 \quad 3.725 e-13 \quad 4.462 e-13 \quad 4.940 e-13 \quad 5.968 e-13$ $\begin{array}{lllllll}8.800 e-02 & 3.284 e-13 & 3.573 e-13 & 3.766 e-13 & 4.471 e-13 & 4.909 e-13 & 5.883 e-13\end{array}$ $9.050 e-02 \quad 3.340 e-13 \quad 3.615 e-13 \quad 3.786 e-13 \quad 4.467 e-13 \quad 4.874 e-13 \quad 5.806 e-13$

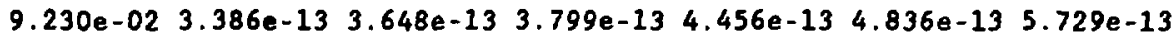


0.600.02 3.4k20.[3 3.635-13 3.016e-13 4.436e-13 4.775e-13 5.6230-13

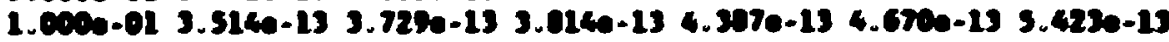

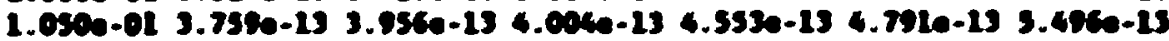
$1.1000-01 \quad 4.1720-13 \quad 4.3630-13 \quad 4.3400-13 \quad 4.9360-13 \quad 5.1400-135.0470-13$ $1.1900-01 \quad 4.5760-13 \quad 4.757 e-13 \quad 4.7460-13 \quad 5.303 e-13 \quad 5.407 e-13 \quad 6.176 e-13$ 1.210e-01 $5.006 e-13 \quad 5.177 e-13 \quad 5.129 e-13 \quad 5.687 e-13 \quad 5.034 e-13 \quad 6.510 e-13$ 1.350e-01 $5.748--13 \quad 5.501 e-13 \quad 5.705 e-13 \quad 0.332 e-13 \quad 6.415 e-13 \quad 7.042 e-13$ $1.490-01 \quad 6.727 e-13 \quad 6.039 e-13 \quad 6.6200-13 \quad 7.132 e-13 \quad 7.105 e-13 \quad 7.640 e-13$ 1.640e-01 $7.706 e-13 \quad 7.709 e-13 \quad 7.479 e-13 \quad 7.975 e-13 \quad 7.057 e-13 \quad 8.329 e-13$ $1.800 e-01 \quad 0.745 e-13 \quad 0.019 e-13 \quad 0.463 e-13 \quad 0.964 e-13 \quad 0.793 e-13 \quad 0.260 e-13$ $1.900 e-01 \quad 9.004 e-13 \quad 9.065 e-13 \quad 9.416 e-13 \quad 9.957 \bullet-13 \quad 9.724 e-13 \quad 1.019 e-12$ 2.100e-01 $1.095 e-12 \quad 1.100 e-12 \quad 1.047 e-12 \quad 1.104 e-12 \quad 1.075 e-12 \quad 1.121 e-12$ 2.390e-01 $1.216 e-12 \quad 1.221 e-12 \quad 1.162 e-12 \quad 1.223 e-12 \quad 1.189 e-12 \quad 1.239 e-12$ 2.630e-01 $1.340 e-12 \quad 1.345 e-12 \quad 1.274 e-12 \quad 1.344 e-12 \quad 1.305 e-12 \quad 1.356 e-12$ 2.900e-01 $1.468 e-12 \quad 1.472 e-12 \quad 1.398 e-12 \quad 1.468 e-12 \quad 1.423 e-12 \quad 1.477 e-12$ 3.190e-01 $1.599 e-12 \quad 1.602 e-12 \quad 1.520 e-12 \quad 1.594 a-12 \quad 1.543 e-12 \quad 1.598 e-12$ 3.510e-01 $1.743 e-12 \quad 1.746 e-12 \quad 1.656 e-12 \quad 1.736 e-12 \quad 1.680 e-12 \quad 1.738 e-12$ 3.860e-01 1.888e-12 $1.891 e-12 \quad 1.793 e-12 \quad 1.879 e-12 \quad 1.817 e-12 \quad 1.879 e-12$ 4.240e-01 2.032e-12 2.035e-12 1.929e-12 $2.021 e-12$ 1.954e-12 2.019e-12 $\begin{array}{lllllll}4.670 e-01 & 2.19 z e-12 & 2.195 e-12 & 2.081 e-12 & 2.179 e-12 & 2.106 e-12 & 2.176 e-12\end{array}$ $\begin{array}{lllllll}5.130 e-01 & 2.354 e-12 & 2.357 e-12 & 2.234 e-12 & 2.339 e-12 & 2.261 e-12 & 2.335 e-12\end{array}$ $\begin{array}{lllllll}5.650 e-01 & 2.597 e-12 & 2.600 e-12 & 2.464 e-12 & 2.579 e-12 & 2.492 e-12 & 2.573 e-12\end{array}$ 6.210e-01 2.869e-12 2.871e-12 2.721e-12 2.848e-12 2.752e-12 2.841e-12 6.830e-01 3.158e-12 $3.160 e-12 \quad 2.995 e-12 \quad 3.134 e-12 \quad 3.028 e-12 \quad 3.126 e-12$ 7.510e-01 3.449e-12 $3.451 e-12 \quad 3.271 e-12 \quad 3.422 e-12 \quad 3.306 e-12 \quad 3.412 e-12$ 8.260e-01 3.731e-12 $3.734 e-12 \quad 3.538 e-12 \quad 3.702 e-12 \quad 3.575 e-12 \quad 3.690 e-1.2$ 9.090e-01 4.039e-12 4.042e-12 $3.830 e-12 \quad 4.007 e-12 \quad 3.869 e-12 \quad 3.994 e-12$ $1.000 e+00 \quad 4.345 e-12 \quad 4.348 e-12 \quad 4.120 e-12 \quad 4.311 e-12 \quad 4.162 e-12 \quad 4.296 e-12$ $\begin{array}{lllllll}1.120 e+00 & 4.757 e-12 & 4.760 e-12 & 4.510 e-12 & 4.719 e-12 & 4.556 e-12 & 4.703 e-12\end{array}$ $1.270 e+00 \quad 5.302 e-125.306 e-12 \quad 5.027 e-12 \quad 5.260 e-12 \quad 5.079 e-12 \quad 5.242 e-12$ $\begin{array}{lllllll}1.420 e+00 & 5.810 e-12 & 5.814 e-12 & 5.509 e-12 & 5.764 e-12 & 5.566 e-12 & 5.745 e-12\end{array}$ $\begin{array}{lllllll}1.600 e+00 & 6.276 e-12 & 6.281 e-12 & 5.953 e-12 & 6.229 e-12 & 6.015 e-12 & 6.209 e-12\end{array}$ $\begin{array}{lllllll}1.800 e+00 & 6.843 e-12 & 6.850 e-12 & 6.493 e-12 & 6.796 e-12 & 6.565 e-12 & 6.779 e-12\end{array}$ $2.030 e+00 \quad 7.383 e-12 \quad 7.394 e-12 \quad 7.012 e-12 \quad 7.343 e-12 \quad 7.096 e-12 \quad 7.330 e-12$ $2.280 e+00 \quad 8.063 e-12 \quad 8.080 e-12 \quad 7.668 e-12 \quad 8.034 e-12 \quad 7.769 e-12 \quad 8.030 e-12$ $2.570 e+00 \quad 8.838 e-12 \quad 8.864 e-12 \quad 8.419 e-12 \quad 8.830 e-12 \quad 8.546 e-12 \quad 8.841 e-12$

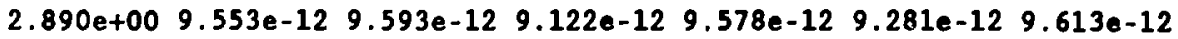
$3.250 e+001.024 e-11 \quad 1.030 e-11 \quad 9.810 e-12 \quad 1.031 e-11 \quad 1.001 e-11 \quad 1.038 e-11$ $3.650 e+001.111 e-11 \quad 1.119 e-11 \quad 1.068 e-11 \quad 1.124 e-11 \quad 1.093 e-11 \quad 1.135 e-11$ 4.110e+00 1.194e-11 $1.205 e-11 \quad 1.152 e-11 \quad 1.216 e-11 \quad 1.185 e-11 \quad 1.233 e-11$ 4.620e+00 1.294e-11 $1.310 \mathrm{e}-11 \quad 1.255 \mathrm{e}-11 \quad 1.328 \mathrm{e}-11 \quad 1.297 \mathrm{e}-11 \quad 1.353 \mathrm{e}-11$ $5.200 e+001.401 e-11 \quad 1.422 e-11 \quad 1.367 e-11 \quad 1.450 e-11 \quad 1.419 e-11 \quad 1.485 e-11$ $5.850 e+001.518 e-11 \quad 1.546 e-11 \quad 1.490 e-11 \quad 1.584 e-11 \quad 1.555 e-11 \quad 1.631 e-11$ $6.580 e+00 \quad 1.649 e-11 \quad 1.684 e-11 \quad 1.628 e-11 \quad 1.736 e-11 \quad 1.709 e-11 \quad 1.797 e-11$ $7.410 e+001.808 e-11 \quad 1.852 e-11 \quad 1.797 e-11 \quad 1.923 e-11 \quad 1.898 e-11 \quad 2.001 e-11$ 


\section{ars}

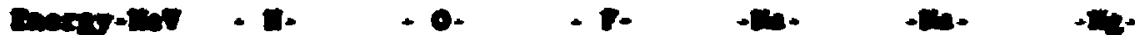

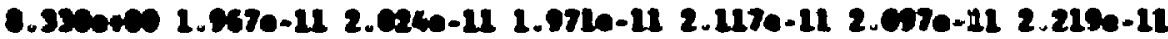

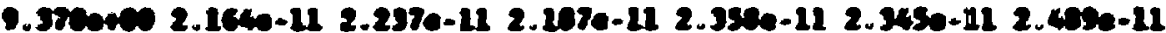

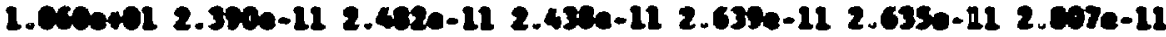

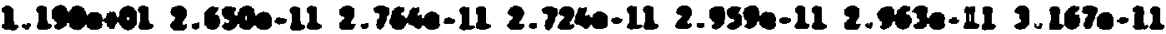

340erel 2.924e-11 3.057e-11 3.036-11 3.306e-11 3.323e-11 3.562e-11

$.000 \mathrm{col}$ 3.735e-11 3.960-11 3.954e-11 4.357e-11 4.414e-11 4.767e-11

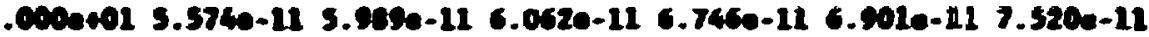

4.000ee01 7.05se-21 8.532e-11 8.714e-11 9.7720-11 $1.0070-110$ 1.203e-10

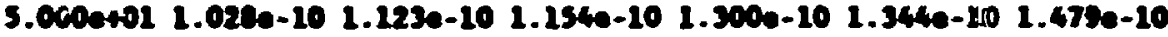

7.500e401 1.472e-10 1.619e-10 $1.671 e-10$ 1.891e-10 $1.963 e-10$ 2.165e-10

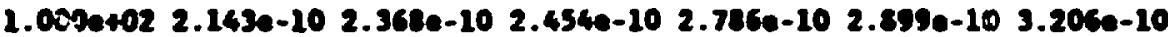




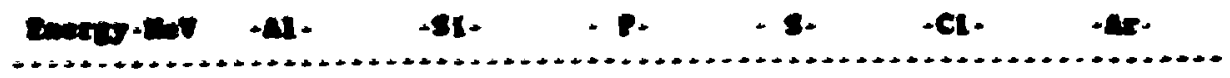

L.000.0\%

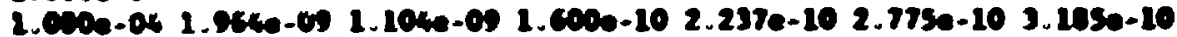

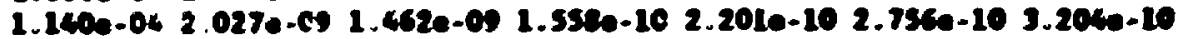

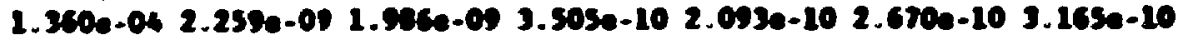

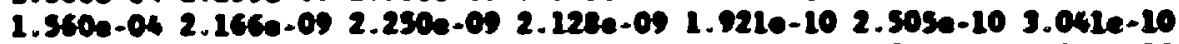

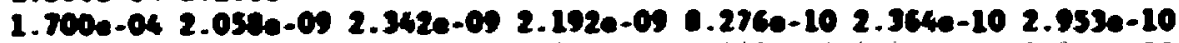

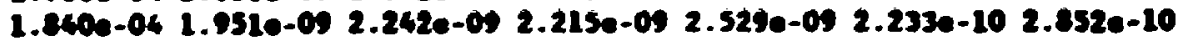
$1.9100-041.8430-052.165 e-052.2410-092.3790-052.1510-102.7400-10$

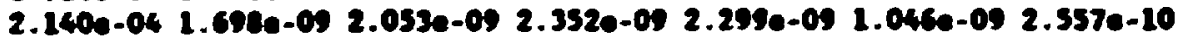
2.240e-04 1.639e-0s 1.934e-0s 2.258e-0s 2.247e-0s 2.26se-0s $1.423 e-10$ 2.410e-04 1.587e-09 1.017e-05 2.149e-09 2.358e-0s 2.287e-0s 2.313e-10 2.470e-04 1.531e-09 1.724e-09 2.061e-09 2.376e-09 2.209e-09 4.164e-10 2.630e-04 1.463e-09 1.674e-09 2.004e-09 2.328e-09 2.168e-09 2.625e-09 2.750e-04 1.362e-09 1.596e-09 $1.916 e-092.248 e-092.194 e-09$ 2.583e-0s 2.820e-04 1.282e-09 1.533e-09 1.844e-09 2.182e-09 2.290e-09 2.284e-09 2.960e-04 1.183e-09 1.454e-09 1.752e-09 2.096e-09 2.237e-09 2.189e-09 3.180e-04 1.060e-09 1.347e-09 1.629e-09 1.977e-09 2.153e-09 2.100e-09 3.550e-04 9.860e-10 1.257e-09 1.524e-09 1.857e-09 2.038e-09 2.174e-09 3.610e-04 9.184e-10 $1.172 e-09 \quad 1.424 e-09 \quad 1.743 e-09 \quad 1.925 e-09$ 2.096e-09 $3.700 e-048.903 e-10 \quad 1.137 e-09$ 1.383e-09 $1.696 e-09 \quad 1.877 e-09 \quad 2.057 e-09$ 3.830e-04 8.456e-10 $1.082 e-09$ 1.3i7e-09 1.619e-09 1.800e-09 1.994e-09 $4.030 e-047.714 e-109.889 e-101.208 e-09 \quad 1.492 e-091.672 e-09 \quad 1.886 e-09$ 4.150e-04 7.248e-10 9.308e-10 1.139e-09 1.411e-09 1.588e-09 1.810e-09 4.300e-04 7.048e-10 9.063e-10 1.109e-09 $1.375 e-09 \quad 1.550 e-09$ 1.766e-09 4.500e-04 6.747e-10 8.691e-10 1.064e-09 1.320e-09 1.491e-09 1.700e-09 4.810e-04 6.222e-10 8.040e-10 9.856e-10 1.224e-09 1.387e-09 1.582e-09 5.160e-04 5.469e-10 7.102e-10 8.722e-10 1.084e-09 1.237e-09 1.412e-09 5.470e-04 5.052e-10 6.569e-10 8.073e-10 1.006e-09 $1.150 e-09$ 1.313e-09 5.550e-04 4.825e-10 6.275e-10 7.715e-10 9.625e-10 1.101e-09 1.258e-09 5.930e-04 4.513e-10 5.869e-10 7.220e-10 9.025e-10 $1.034 e-09$ 1.182e-09 5.980e-04 4.192e-10 $5.453 e-10 \quad 6.712 e-10 \quad 8.409 e-10 \quad 9.644 e-10 \quad 1.103 e-09$ $6.250 e-04 \quad 4.042 e-10 \quad 5.260 e-10 \quad 6.477 e-10 \quad 8.120 e-10 \quad 9.319 e-10 \quad 1.066 e-09$ $6.540 e-04 \quad 3.829 e-10 \quad 4.989 e-10 \quad 6.148 e-10 \quad 7.709 e-10 \quad 8.857 e-10 \quad 1.014 e-09$ $6.750 e-04 \quad 3.611 e-10 \quad 4.712 e-10 \quad 5.810 e-10 \quad 7.287 e-10 \quad 8.382 e-10 \quad 9.596 e-10$ $6.980 e-04 \quad 3.396 e-10 \quad 4.437 e-10 \quad 5.475 e-10 \quad 6.869 e-10 \quad 7.911 e-10 \quad 9.060 e-10$ $7.110 e-043.233 e-10 \quad 4.229 e-10 \quad 5.222 e-10 \quad 6.552 e-10 \quad 7.555 e-10 \quad 8.653 e-10$ 7.250e-04 3.164e-10 $4.139 e-10 \quad 5.112 e-10 \quad 6.417 e-10 \quad 7.402 e-10 \quad 8.477 e-10$ $7.710 e-042.992 e-10 \quad 3.916 e-10 \quad 4.840 e-10 \quad 6.079 e-10 \quad 7.022 e-10 \quad 8.040 e-10$ $7.940 e-042.769 e-10 \quad 3.626 e-10 \quad 4.486 e-10 \quad 5.640 e-10 \quad 6.528 e-10 \quad 7.470 e-10$ $8.180 e-042.624 e-10 \quad 3.437 e-10 \quad 4.254 e-10 \quad 5.354 e-10 \quad 6.203 e-10 \quad 7.098 e-10$ $8.380 e-042.535 e-10 \quad 3.323 e-10 \quad 4.111 e-10 \quad 5.176 e-10 \quad 5.999 e-10 \quad 6.866 e-10$ $8.600 e-042.445 e-10 \quad 3.207 e-10 \quad 3.965 e-10 \quad 4.996 e-10 \quad 5.792 e-10 \quad 6.631 e-10$ $\begin{array}{llllllll}8.980 e-04 & 2.302 e-10 & 3.023 e-10 & 3.734 e-10 & 4.711 e-10 & 5.464 e-10 & 6.259 e-10\end{array}$ $9.200 e-042.171 e-10 \quad 2.862 e-10 \quad 3.537 e-10 \quad 4.467 e-10 \quad 5.158 e-10 \quad 5.926 e-10$ $9.400 e-042.112 e-10 \quad 2.804 e-10 \quad 3.478 e-10 \quad 4.391 e-10 \quad 5.013 e-10 \quad 5.798 e-10$ $9.560 e-042.058 e-10 \quad 2.750 e-10 \quad 3.422 e-10 \quad 4.321 e-10 \quad 4.879 e-10 \quad 5.678 e-10$ 


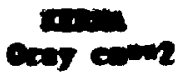

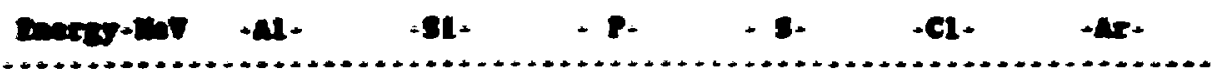

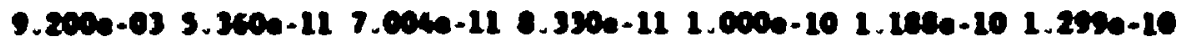
9.400-03 $5.2020-11 \quad 6.6700-11 \quad 7.960 e-11 \quad 9.5350-11 \quad 1.1350-101.2600-10$

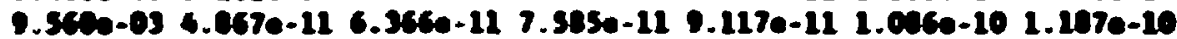

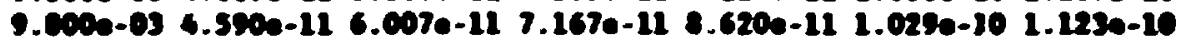

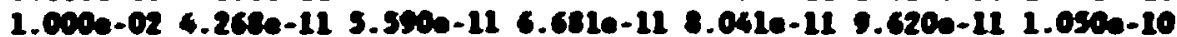
$1.0400-02 \quad 4.0790-115.3440-11 \quad 0.3930-11 \quad 7.6990-12 \quad 9.2250-11 \quad 1.0060-10$ 1.000e-02 3.940e-11 $5.229 e-11 \quad 6.256 e-11 \quad 7.539 e-11 \quad 0.037 e-11 \quad 9.057 e-11$ 1.110e-02 3.89e--11 $5.109 e-11 \quad 6.126 e-11 \quad 7.371 e-11$ e.e39e-11 e.641e-11 1.190e-02 3.722e-11 $4.076 e-11$ 5.043e-11 $7.047 e-11$ e.456e-11 $9.223 e-11$ 1.270e-02 3.405e-11 $4.464 e-115.354 e-11 \quad 6.466 e-11 \quad 7.769 e-11 \quad 8.471 e-11$ 1.300e-02 3.147e-11 4.127e-11 4.954e-11 $5.990 e-11 \quad 7.206 e-11 \quad 7.055 e-11$ 1.390e-02 2.819e-11 $3.697 e-11 \quad 4.445 e-11 \quad 5.383 e-11 \quad 6.409 e-11 \quad 7.071 e-11$ 1.470e-02 2.207e-11 3.001e-11 3.621e-11 4.401e-11 $5.325 e-11 \quad 5.790 e-11$ 1.520e-02 1.852e-11 2.432e-11 $2.945 e-11 \quad 3.596 e-11 \quad 4.370 e-11 \quad 4.754 e-11$ 1.570e-02 $1.744 e-11 \quad 2.290 e-11 \quad 2.776 e-11 \quad 3.394 e-11 \quad 4.129 e-11 \quad 4.492 e-11$ 1.640e-02 1.675e-11 $2.200 e-11 \quad 2.669 e-11 \quad 3.265 e-11 \quad 3.973 e-11 \quad 4.322 e-11$ 1.700e-02 1.591e-11 2.086e-11 $2.535 e-11 \quad 3.104 e-11 \quad 3.779 e-11 \quad 4.111 e-11$ 1.720e-02 $1.533 e-112.012 e-11 \quad 2.443 e-11 \quad 2.994 e-11 \quad 3.646 e-11 \quad 3.966 e-11$ 1.810e-02 1.444e-11 $1.896 e-11 \quad 2.303 e-11 \quad 2.826 e-11 \quad 3.443 e-11 \quad 3.745 e-11$ $1.900 e-02 \quad 1.283 e-11 \quad 1.683 e-11 \quad 2.048 e-11 \quad 2.519 e-11 \quad 3.071 e-11 \quad 3.341 \bullet-11$ 1.970e-02 1.121e-11 $1.470 e-11 \quad 1.791 e-11 \quad 2.210 e-11 \quad 2.697 e-11 \quad 2.933 e-11$ 2.010e-02 1.001e-11 $1.313 e-11 \quad 1.602 e-11 \quad 1.983 e-11 \quad 2.422 e-11 \quad 2.634 e-11$ 2.100e-02 9.629e-12 $1.262 e-11 \quad 1.541 e-11 \quad 1.909 e-11 \quad 2.332 e-11 \quad 2.537 e-11$ 2.170e-02 9.407e-12 $1.233 e-11 \quad 1.505 e-11 \quad 1.866 e-11 \quad 2.279 e-11 \quad 2.479 e-11$ 2.230e-02 9.194e-12 $1.204 e-11 \quad 1.471 e-11 \quad 1.824 e-11 \quad 2.228 e-11 \quad 2.424 e-11$ 2.310e-02 8.929e-12 $1.169 e-11 \quad 1.428 e-11 \quad 1.773 e-11 \quad 2.165 e-11 \quad 2.356 e-11$ 2.550e-02 8.183e-12 1.071e-11 $1.309 e-11$ 1.627e-11 1.987e-11 2.162e-11 2.920e-02 6.269e-12 8.185e-12 $1.002 e-11 \quad 1.253 e-11 \quad 1.529 e-11 \quad 1.665 e-11$ 3.320e-02 4.054e-12 5.263e-12 6.461e-12 8.181e-12 9.967e-12 $1.086 e-11$ 3.750e-02 3.336e-12 4.317e-12 5.298e-12 $6.737 e-12 \quad 3.189 e-12 \quad 8.932 e-12$ 4.200e-02 2.437e-12 $3.133 e-12 \quad 3.841 e-12 \quad 4.920 e-12 \quad 5.955 e-12 \quad 6.504 e-12$ 4.740e-02 1.973e-12 2.520e-12 3.083e-12 $3.964 e-12 \quad 4.777 e-12 \quad 5.220 e-12$ 4.850e-02 $1.695 e-12 \quad 2.155 e-12 \quad 2.629 e-12 \quad 3.389 e-12 \quad 4.068 e-12 \quad 4.446 e-12$ $5.020 e-02 \quad 1.556 e-12 \quad 1.971 e-12 \quad 2.401 e-12 \quad 3.099 e-12 \quad 3.712 e-12 \quad 4.057 e-12$ 5.500e-02 $1.403 e-12 \quad 1.768 e-12 \quad 2.148 e-12 \quad 2.774 e-12 \quad 3.311 e-12 \quad 3.618 e-12$ 6.100e-02 $1.184 e-12 \quad 1.479 e-12 \quad 1.785 e-12 \quad 2.304 e-12 \quad 2.731 e-12 \quad 2.983 e-12$ 6.750e-02 $1.039 e-12 \quad 1.285 e-12 \quad 1.539 e-12 \quad 1.982 e-12 \quad 2.333 e-12 \quad 2.544 e-12$ 6.950e-02 9.796e-13 1.205e-12 $1.436 e-12$ 1.844e-12 2.161e-12 2.354e-12 $\begin{array}{lllllll}7.350 e-02 & 9.266 e-13 & 1.133 e-12 & 1.345 e-12 & 1.724 e-12 & 2.012 e-12 & 2.189 e-12\end{array}$

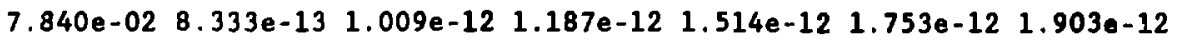
8.070e-02 $7.482 e-13 \quad 8.958 e-13 \quad 1.043 e-12 \quad 1.324 e-12 \quad 1.520 e-12 \quad 1.645 e-12$ $\begin{array}{lllllll}8.550 e-02 & 7.228 e-13 & 8.603 e-13 & 9.963 e-13 & 1.259 e-12 & 1.438 e-12 & 1.554 e-12\end{array}$ 8.800e-02 $7.064 e-13 \quad 8.364 e-13 \quad 9.634 e-13 \quad 1.213 e-12 \quad 1.379 e-12 \quad 1.487 e-12$ $9.050 e-02 \quad 6.925 e-13 \quad 8.167 e-13 \quad 9.369 e-13 \quad 1.176 e-12 \quad 1.332 e-12 \quad 1.434 e-12$ $9.230 e-026.791 e-13 \quad 7.978 e-13 \quad 9.117 e-13 \quad 1.140 e-12 \quad 1.288 e-12 \quad 1.385 e-12$ 


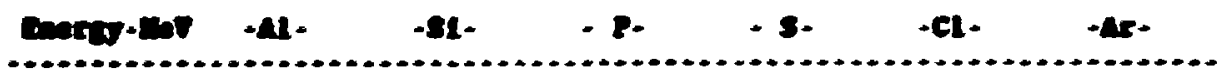

9.6000-02 6.566-13 7.707e-13 8.75e-13 1.000e-12 $1.226 e-12$ 1.315e-12 1.0000-01 6.204e-13 $7.27 e-138.195 e-13$ 1.012e-12 $1.129 e-12$ 1.206e-12

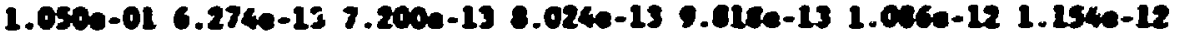
1.100-01 6.550e-13 $7.5050-138.2500-13 \quad 1.0050-121.1040-12 \quad 1.167 e-12$ 1.25e-01 6.07ce-13 $7.7760-13 \quad 0.5150-13 \quad 1.023 e-12 \quad 1.115 e-12 \quad 1.173 e-12$ 1.210e-01 $7.160 e-13 \quad 0.032 e-13 \quad 8.714 e-13$ l.036e-12 $1.121 e-12 \quad 1.173 e-12$ 1.350e-01 $7.5760-13 \quad .3030-13 \quad 8.9380-13 \quad 1.0430-12 \quad 1.1120-12 \quad 1.140-12$ 1.450-01 $7.977 \bullet-13 \quad 0.651 e-13 \quad 0.989 \bullet-13 \quad 1.016 e-12 \quad 1.057 e-12 \quad 1.072 e-12$ 1.640e-01 $\quad 0.505 e-13 \quad 9.091 e-13 \quad 9.257 e-13 \quad 1.022 e-12 \quad 1.034 e-12 \quad 1.034 e-12$ 1.000e-01 $9.379 e-13 \quad 9.958 e-13 \quad 1.005 e-12 \quad 1.056 e-12 \quad 1.102 e-12 \quad 1.004 e-12$ 1.980-01 $1.021 e-12 \quad 1.077 e-12 \quad 1.077 e-12 \quad 1.158 e-12 \quad 1.152 e-12 \quad 1.125 e-12$ 2.100e-01 $1.116 e-12 \quad 1.171 e-12 \quad 1.161 e-12 \quad 1.236 e-12 \quad 1.218 e-12 \quad 1.179 e-12$ 2.390e-01 $1.229 e-12 \quad 1.286 e-12 \quad 1.272 e-12 \quad 1.347 e-12 \quad 1 / 323 e-12 \quad 1.275 e-12$ 2.630e-01 $1.341 e-12 \quad 1.401 e-12 \quad 1.381 e-12 \quad 1.456 e-12 \quad 1.423 e-12 \quad 1.366 e-12$ 2.900e-01 $1.455 e-12 \quad 1.516 e-12 \quad 1.489 e-12 \quad 1.560 e-12 \quad 1.518 e-12 \quad 1.450 e-12$ 3.190e-01 $1.570 e-12 \quad 1.632 e-12 \quad 1.597 e-12 \quad 1.664 e-12 \quad 1.613 e-12 \quad 1.532 e-12$ 3.510e-01 $1.705 e-12 \quad 1.772 e-12 \quad 1.731 e-12 \quad 1.800 e-12 \quad 1.742 e-12 \quad 1.652 e-12$ 3.860e-01 $1.841 e-12 \quad 1.911 e-12 \quad 1.866 e-12 \quad 1.936 e-12 \quad 1.870 e-12 \quad 1.770 e-12$ 4.240e-01 $1.977 e-12 \quad 2.050 e-12 \quad 1.999 e-12 \quad 2.070 e-12 \quad 1.997 e-12 \quad 1.887 e-12$ $\begin{array}{llllllll}4.670 e-01 & 2.129 e-12 & 2.207 e-12 & 2.151 e-12 & 2.225 e-12 & 2.145 e-12 & 2.024 e-12\end{array}$ $\begin{array}{lllllll}5.130 e-01 & 2.283 e-12 & 2.366 e-12 & 2.304 e-12 & 2.381 e-12 & 2.294 e-12 & 2.163 e-12\end{array}$ $\begin{array}{lllllll}5.650 e-01 & 2.515 e-12 & 2.606 e-12 & 2.536 e-12 & 2.619 e-12 & 2.522 e-12 & 2.376 e-12\end{array}$ $\begin{array}{lllllll}6.210 e-01 & 2.775 e-12 & 2.874 e-12 & 2.797 e-12 & 2.8 B 7 e-12 & 2.778 e-12 & 2.616 e-12\end{array}$ 6.830e-01 $3.053 e-12 \quad 3.161 e-12 \quad 3.075 e-12 \quad 3.173 e-12 \quad 3.053 e-12 \quad 2.873 e-12$ $7.510 e-02 \quad 3.332 e-12 \quad 3.450 e-12 \quad 3.355 e-12 \quad 3.461 e-12 \quad 3.329 e-12 \quad 3.133 e-12$ 8.260e-01 3.6r 3e-12 3.730e-12 $3.626 e-12 \quad 3.740 e-12 \quad 3.596 e-12 \quad 3.383 e-12$ 9.090e-01 3.899e-12 4.036e-12 $3.923 e-12$ 4.045e-12 $3.890 e-12 \quad 3.659 e-12$ $1.000 e+00 \quad 4.194 e-12 \quad 4.341 e-12 \quad 4.219 e-12 \quad 4.350 e-12 \quad 4.182 e-12 \quad 3.933 e-12$ $1.120 e+00 \quad 4.590 e-12 \quad 4.751 e-12 \quad 4.617 e-12 \quad 4.760 e-12 \quad 4.576 e-12 \quad 4.302 e-12$ $1.270 e+005.117 e-125.295 e-125.146 e 125.305 e-125.100 e-12 \quad 4.795 e-12$ $1.420 e+00 \quad 5.608 e-125.804 e-12 \quad 5.640 e-12 \quad 5.815 e-12 \quad 5.589 e-12 \quad 5.255 e-12$ $\begin{array}{lllllll}1.600 e+00 & 6.061 e-12 & 6.274 e-12 & 6.097 e-12 & 6.286 e-12 & 6.043 e-12 & 5.682 e-12\end{array}$ $\begin{array}{lllllll}1.800 e+00 & 6.619 e-12 & 6.853 e-12 & 6.662 e-12 & 6.871 e-12 & 6.606 e-12 & 6.213 e-12\end{array}$ 2.030e+00 7.161e-12 7.417e-12 $7.213 e-12 \quad 7.442 e-12 \quad 7.159 e-12 \quad 6.735 e-12$ $2.280 e+007.850 e-12 \quad 8.135 e-12 \quad 7.917 e-12 \quad 8.173 e-12 \quad 7.867 e-12 \quad 7.405 e-12$ $2.570 e+008.649 e-128.972 e-12 \quad 8.738 e-12 \quad 9.029 e-12 \quad 8.698 e-12 \quad 8.195 e-12$ $2.890 e+009.415 e-12 \quad 9.777 e-12 \quad 9.533 e-12 \quad 9.861 e-12 \quad 9.511 e-12 \quad 8.970 e-12$ $3.250 e+001.019 e-11 \quad 1.059 e-11 \quad 1.034 e-11 \quad 1.071 e-11 \quad 1.034 e-11 \quad 9.771 e-12$ $3.650 e+001.116 \mathrm{e}-11 \quad 1.162 \mathrm{e}-11 \quad 1.136 \mathrm{e}-11 \quad 1.179 \mathrm{e}-11 \quad 1.140 \mathrm{e}-11 \quad 1.078 \mathrm{e}-11$ 4.110e+00 $1.214 \mathrm{e}-11 \quad 1.267 \mathrm{e}-11 \quad 1.241 \mathrm{e}-11 \quad 1.290 \mathrm{e}-11 \quad 1.250 \mathrm{e}-11 \quad 1.185 \mathrm{e}-11$ 4.620e+00 1.335e-11 $1.396 e-11 \quad 1.371 e-11 \quad 1.428 e-11 \quad 1.386 e-11 \quad 1.316 e-11$ $5.200 e+00 \quad 1.468 e-11 \quad 1.540 e-11 \quad 1.515 e-11 \quad 1.582 e-11 \quad 1.540 e-11 \quad 1.465 e-11$ $5.850 e+001.617 e-11 \quad 1.699 e-11 \quad 1.677 e-11 \quad 1.754 e-11 \quad 1.711 e-11 \quad 1.632 e-11$ $6.580 \mathrm{e}+00 \quad 1.786 \mathrm{e}-11 \quad 1.882 \mathrm{e}-11 \quad 1.861 \mathrm{e}-11 \quad 1.952 \mathrm{e}-11 \quad 1.908 \mathrm{e}-11 \quad 1.823 \mathrm{e}-11$ $7.410 e+001.995 e-112.107 e-112.089 e-112.196 e-112.152 e-112.061 e-11$ 


\section{a \\ ats ande}

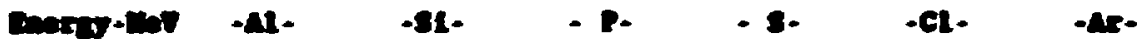

C.3390400 $2.219 a-112.3510-112.337 e-112.4640-112.426 e-112.3240-11$

-.370e+00 $2.497 e-112.653 e-112.6460-112.797 e-112.7940-112.651 e-11$

1.0600n01 $2.026 e-113.0130-113.013 e-113.196-113.1540-113.0440-11$

$1.15004013 .1960-11$ 3.416e-11 3.425e-11 3.638e-11 3.601e-11 3.412e-21

1.3400401 3.c05e-11 3.053e-11 3.cese-11 4.135e-11 4.100e-11 3.972e-21

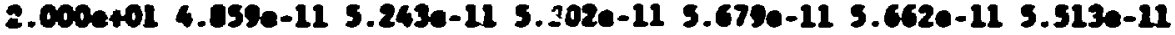

3.000e+01 $7.726 e-11 \quad 0.397 \bullet-11 \quad 0.5500-11 \quad 9.216 e-11 \quad 9.239 a-11 \quad 9.0430-11$

4.000e+01 $1.1390-10 \quad 1.2440-10 \quad 1.2720-10 \quad 1.376 \bullet-10 \quad 1.305 e-10 \quad 1.3600-10$

5.000e+01 $1.532 e-10 \quad 1.677 e-10 \quad 1.719 e-10 \quad 1.063 e-10 \quad 1.078 e-10 \quad 1.046 e-10$

7.500e401 2.250e-10 2.459a-10 $2.536 e-10 \quad 2.754 e-10 \quad 2.700 e-10 \quad 2.734 e-10$

1.000e+02 $3.337 e-103.659 e-103.773 e-10+.103 e-10 \quad 4.146 e-10 \quad 4.05 e--10$ 


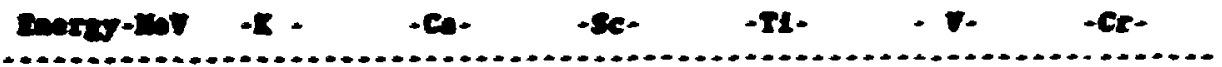

\subsection{0e-04}

$1.0200-043.523 e-103.012 e-10 \quad 4.3050-104.762 e-105.335 e-10 \quad 7.050 e-10$

$1.1400-043.583 e-103.497 e-104.3460-104.842 e-10 \quad 5.411 e-107.1110-10$

$1.3600-043.627 e-103.993 e-104.456 e-104.004 e-10 \quad 5.430 e-107.053 e-10$

$1.560 e-04 \quad 3.551 e-103.996 e-10 \quad 4.3510-10 \quad 4.750 e-10 \quad 5.230=-10 \quad 6.640 e-10$

$1.700 e-043.482 e-103.962 e-10 \quad 4.331 e-10 \quad 4.667 e-10 \quad 5.119 e-10 \quad 6.421 e-10$

$1.0400-043.397 e-103.093 e-104.249 e-104.579 e-105.014 e-10 \quad 5.247 e-10$

1.1210e-04 3.296e-10 3.000e-10 4.143e-10 4.465e-10 4.882e-10 $6.044 a-10$

$2.140 e-043.150 e-103.664 e-103.929 e-104.300 e-104.694 e-10 \quad 5.762 e-10$

2.240e-04 3.093e-10 3.620e-10 3.942e-10 4.253e-10 4.640e-10 5.674e-10

$2.410 e-043.031 e-10 \quad 3.567 e-103.845 e-10 \quad 4.194 e-10 \quad 4.578 e-10 \quad 5.582 e-10$

2.470e-04 2.958e-10 3.499e-10 3.812e-10 4.119e-10 4.496e-10 5.468e-10

$2.630 e-042.866 e-10 \quad 3.410 e-10 \quad 3.716 e-10 \quad 4.018 e-10 \quad 4.387 e-10 \quad 5.319 e-10$

2.750e-04 2.723e-10 $3.268 e-10 \quad 3.563 e-10 \quad 3.858 e-10 \quad 4.212 e-10 \quad 5.085 e-10$

2. 820e-04 2.608e-10 $3.152 e-10 \quad 3.438 e-10 \quad 3.726 e-10 \quad 4.069 e-10 \quad 4.895 e-10$

$2.960 e-04 \quad 3.915 e-10 \quad 3.004 e-10 \quad 3.278 e-10 \quad 3.558 e-10 \quad 3.886 e-10 \quad 4.653 e-10$

3.180e-04 2.886e-09 2.798e-10 3.075e-10 3.344e-10 3.654e-10 4.348e-10

3.550e-04 2.282e-09 6.441e-10 2.937e-10 3.199e-10 $3.500 e-10 \quad 4.152 e-10$

3.610e-04 2.139e-09 2.230e-09 2.801e-10 3.056e-10 3.346e-10 3.958e-10

3.700e-04 2.091e-09 2.267e-09 2.742e-10 2.994e-10 3.279e-10 3.875e-10

3.830e-04 2.130e-09 2.264e-09 2.648e-10 2.893e-10 3.171e-10 3.741e-10

4.030e-04 2.210e-09 2.191e-09 2.487e-10 2.723e-10 2.988e-10 3.514e-10

4.150e-04 2.164e-09 2.112e-09 1.757e-09 2.602e-10 2.858e-10 3.371e-10

4.300e-04 2.117e-09 2.037e-09 2.140e-09 2.534e-10 $2.783 e-10 \quad 3.308 e-10$

4.500e-04 2.045e-09 2.113e-09 2.083e-09 2.431e-10 2.671e-10 3.211e-10

4.810e-04 1.916e-09 2.112e-09 $1.952 e-09 \quad 1.386 e-09 \quad 2.523 e-10 \quad 3.034 e-10$

5.160e-04 1.728e-09 1.946e-09 1.918e-09 1.947e-09 2.534e-10 2.771e-10

5.470e-04 $1.613 e-091.832 e-09 \quad 1.936 e-09 \quad 1.818 e-09 \quad 1.478 e-09 \quad 2.597 e-10$

5.550e-04 $1.548 e-09 \quad 1.764 e-09 \quad 1.848 e-09 \quad 1.735 e-09 \quad 1.726 e-09 \quad 2.494 e-10$

5.930e-04 1.456e-09 $1.667 e-09 \quad 1.757 e-09 \quad 1.846 e-09 \quad 1.790 e-09 \quad 8.883 e-10$

5.980e-04 1.363e-09 1.568e-09 1.679e-09 1.821e-09 1.712e-09 2.023e-09

6.250e-04 1.319e-09 1.521e-09 1.635e-09 1.774e-09 1.652e-09 2.002e-09

$6.540 e-041.257 e-091.454 e-091.563 e-09 \quad 1.700 e-09 \quad 1.789 e-09 \quad 1.881 e-09$

$6.750 e-041.192 e-091.384 e-091.488 e-09 \quad 1.623 e-09 \quad 1.742 e-09 \quad 1.758 e-09$

6.980e-04 1.129e-09 1.315e-09 1.413e-09 1.546e-09 1.667e-09 1.708e-09

7.110e-04 1.080e-09 1.261e-09 $1.356 e-09 \quad 1.486 e-09 \quad 1.609 e-09 \quad 1.857 e-09$

$7.250 e-041.059 e-091.237 e-09$ 1.331e-09 $1.459 e-09$ 1.581e-09 1.818e-09

7.710e-04 1.005e-09 1.176e-09 $1.267 e-09$ 1.390e-09 $1.510 e-09 \quad 1.723 e-09$

7.940e-04 9.356e-10 $1.096 e-09 \quad 1.184 e-09 \quad 1.300 e-09 \quad 1.416 e-09 \quad 1.625 e-09$

8.180e-04 8.899e-10 1.044e-09 $1.129 e-091.241 e-091.355 e-091.563 e-09$

8.380e-04 8.612e-10 1.011e-09 $1.094 \mathrm{e}-09$ 1.204e-09 $1.316 \mathrm{e}-09 \quad 1.520 \mathrm{e}-09$

8.600e-04 8.320e-10 9.775e-10 $1.058 e-09 \quad 1.167 e-09 \quad 1.277 e-09 \quad 1.476 e-09$

8.980e-04 7.856e-10 9.242e-10 $1.001 e-09 \quad 1.107 e-09 \quad 1.214 e-09 \quad 1.405 e-09$

$9.200 e-047.435 e-10 \quad 8.773 e-10 \quad 9.505 e-10 \quad 1.058 e-09 \quad 1.160 e-09 \quad 1.344 e-09$

$9.400 e-047.254 e-10 \quad 8.604 e-10 \quad 9.314 e-10 \quad 1.045 e-09 \quad 1.144 e-09 \quad 1.327 e-09$

$9.560 e-047.088 e-10 \quad 8.447 e-10 \quad 9.137 e-10 \quad 1.033 e-09 \quad 1.129 e-09 \quad 1.310 e-09$

Page -16- 


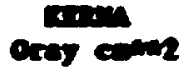

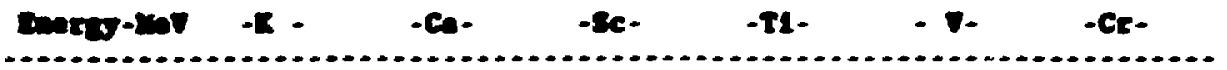

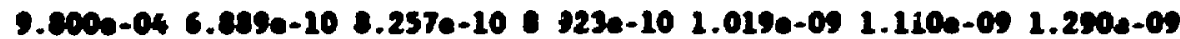

1.500-04 6.705e-10 0.056e-10 e.729e-10 1.005e-0s 1.054e-0s 1.271e-0s

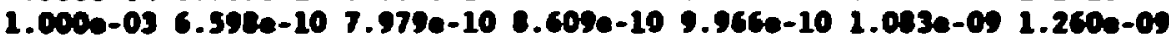

1.000e-03 6.446e-10 7.804e-10 $8.433 e-10 \quad 9.777 e-10 \quad 1.062 e-03 \quad 1.236 e-09$

1.140e-03 6.223e-10 7.536e-10 $\quad .162 e-10 \quad 9.439-10$ i.026e-0s $1.194 e-09$

$1.360 e-03 \quad 5.491 e-10 \quad 6.646 e-10 \quad 7.254 e-10 \quad 0.332 e-10 \quad 9.032 e-10 \quad 1.055 e-03$

1.566e-03 3.907e-10 4.721e-10 5.270e-10 5.934e-10 $6.515 e-10 \quad 7.541 e-10$

$1.700 e-03 \quad 3.223 e-10 \quad 3.891 e-10 \quad 4.396 e-10 \quad 4.899 e-10 \quad 5.401 e-10 \quad 6.240 e-10$

$1.040 e-03 \quad 2.092 e-10 \quad 3.491 e-10 \quad 3.958 e-10 \quad 4.399 e-10 \quad 4.858 \cdot-10 \quad 5.607 e-10$

$1.910 e-03 \quad 2.584 e-103.118 e-103.546 e-10 \quad 3.934 e-10 \quad 4.350 e-10 \quad 5.018 e-10$

2.140e-03 2.191e-10 2.643e-10 3.023e-10 $3.339 e-103.702 e-10 \quad 4.264 e-10$

2.240e-03 2.049e-10 2.472e-10 2.830e-10 $3.125 e-10 \quad 3.468 e-10 \quad 3.993 e-10$

2.410e-03 i.949e-10 2.352e-10 2.692e-10 2.974e-10 $3.303 e-10 \quad 3.801 e-10$

2.470e-03 $1.846 e-102.228 e-10 \quad 2.550 e-10 \quad 2.818 e-10 \quad 3.131 \bullet-10 \quad 3.603 e-10$

2.630e-03 $1.727 e-102.084 e-10 \quad 2.385 e-10 \quad 2.637 e-10 \quad 2.932 e-10 \quad 3.373 \bullet-10$

2.750e-03 1.552e-10 $1.874 e-10 \quad 2.144 e-10 \quad 2.373 e-10 \quad 2.641 e-10 \quad 3.037 e-10$

2.820e-03 $1.418 e-101.713 e-10 \quad 1.959 e-10 \quad 2.170 e-10 \quad 2.417 e-10 \quad 2.778 e-10$

$2.960 e-031.253 e-101.514 e-10 \quad 1.731 e-10 \quad 1.920 e-10 \quad 2.142 e-10 \quad 2.460 e-10$

3.180e-03 $1.044 e-10 \quad 1.273 e-10 \quad 1.454 e-10 \quad 1.617 e-10 \quad 1.803 e-10 \quad 2.074 e-10$

3.550e-03 9.036e-11 $1.152 e-10 \quad 1.311 e-10 \quad 1.463 e-10 \quad 1.637 e-10 \quad 1.877 e-10$

3.610e-03 $1.134 e-10 \quad 1.046 e-10 \quad 1.186 e-10 \quad 1.330 e-10 \quad 1.488 e-10 \quad 1.706 e-10$

3.700e-03 6.684e-10 $1.003 e-10 \quad 1.136 e-10 \quad 1.275 e-10 \quad 1.428 e-10 \quad 1.636 e-10$

3.830e-03 6.446e-10 $9.347 e-11 \quad 1.056 e-10 \quad 1.189 e-10 \quad 1.332 e-10 \quad 1.526 e-10$

$\begin{array}{lllllll}4.030 e-03 & 6.044 e-10 & 8.227 e-11 & 9.253 e-11 & 1.048 e-10 & 1.175 e-10 & 1.345 e-10\end{array}$

4.150e-03 5.755e-10 $6.448 e-10 \quad 8.374 e-11 \quad 9.613 e-11 \quad 1.079 e-10 \quad 1.235 e-10$

4.300e-03 5.587e-10 6.627e-10 7.930e-11 9.267e-11 1.041e-1ú 1.192e-10

4.500e-03 5.335e-10 6.320e-10 9.252e-11 $8.754 e-11 \quad 9.851 e-11 \quad 1.128 e-10$

$4.810 e-03 \quad 4.897 e-10 \quad 5.787 e-10 \quad 5.692 e-10 \quad 7.876 e-11 \quad 8.891 e-11 \quad 1.017 e-10$

5.160e-03 4.271e-10 5.025e-10 5.147e-10 3.395e-10 $7.513 e-11 \quad 8.618 e-11$

$5.470 e-03 \quad 3.917 e-10 \quad 4.595 e-10 \quad 4.770 e-10 \quad 5.239 e-10 \quad 7.475 e-11 \quad 7.777 e-11$

5.550e-03 3.724e-10 4.361e-10 4.544e-10 4.987e-10 5.406e-10 7.331e-11

5.930e-03 $3.458 e-10 \quad 4.041 e-10 \quad 4.234 e-10 \quad 4.639 e-10 \quad 5.089 e-10 \quad 6.724 e-11$

$5.980 e-03 \quad 3.187 e-10 \quad 3.713 e-10 \quad 3.917 e-10 \quad 4.283 e-10 \quad 4.762 e-10 \quad 6.107 e-11$

$6.250 e-03 \quad 3.086 e-10 \quad 3.593 e-10 \quad 3.799 e-10 \quad 4.152 e-10 \quad 4.629 e-10 \quad 5.043 e-10$

$6.540 e-03 \quad 2.975 e-10 \quad 3.461 e-10 \quad 3.667 e-10 \quad 4.007 e-10 \quad 4.462 e-10 \quad 5.008 e-10$

$6.750 e-03 \quad 2.858 e-10 \quad 3.323 e-10 \quad 3.528 e-10 \quad 3.853 e-10 \quad 4.287 e-10 \quad 4.815 e-10$

$6.980 e-03 \quad 2.740 e-10 \quad 3.184 e-10 \quad 3.387 e-10 \quad 3.698 e-10 \quad 4.110 e-10 \quad 4.621 e-10$

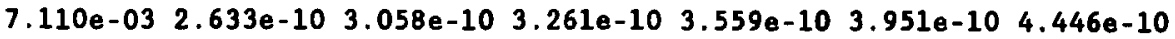

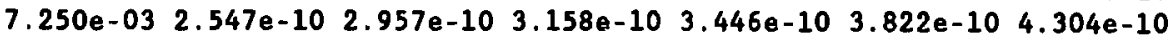

$\begin{array}{llllll}7.710 e-032.335 e-10 & 2.708 e-10 & 2.905 e-10 & 3.168 e-10 & 3.505 e-10 & 3.955 e-10\end{array}$

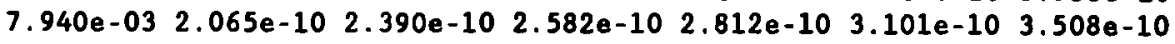

$\begin{array}{lllllll}8.180 e-03 & 1.907 e-10 & 2.204 e-10 & 2.392 e-10 & 2.603 e-10 & 2.864 e-10 & 3.247 e-10\end{array}$

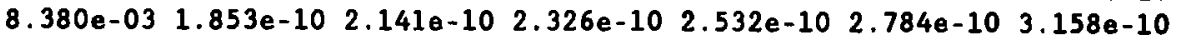

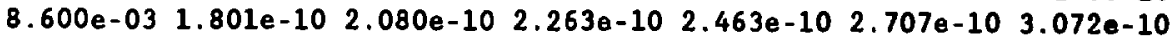

$8.980 e-03 \quad 1.717 e-10 \quad 1.984 e-102.161 e-102.352 e-10 \quad 2.583 e-10 \quad 2.934 e-10$ 
Iin

Cras one?

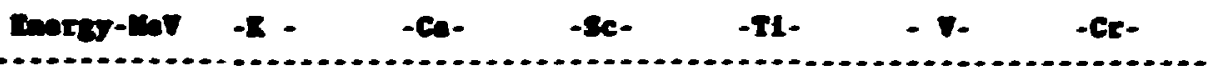

$9.200 e-03 \quad 1.6240-101.877 e-10 \quad 2.046 e-10 \quad 2.230 e-10 \quad 2.446 e-10 \quad 2.792 e-10$ 9.400--03 $1.553 e-10 \quad 1.794 e-10 \quad 1.961 e-10 \quad 2.135 e-10 \quad 2.3410-10 \quad 2.664 e-10$ 9.560e-03 $1.494 e-10 \quad 1.720 e-10 \quad 1.092 e-10 \quad 2.049 e-10 \quad 2.245 e-10 \quad 2.557 e-10$ 9.600e-03 $1.412 e-10 \quad 1.631 e-10 \quad 1.798 e-10 \quad 1.946 e-10 \quad 2.132 e-10 \quad 2.431 e-10$ 1.000e-02 $1.323 e-10 \quad 1.529 e-10 \quad 1.680 e-10 \quad 1.829 e-10 \quad 2.000 e-10 \quad 2.283 e-10$ 1.040e-02 $1.270 e-101.467 \bullet-10 \quad 1.615 e-10 \quad 1.759 a-10 \quad 1.922 e-10 \quad 2.196 e-10$ 1.080e-02 $1.245 e-101.439 e-10 \quad 1.583 e-10 \quad 1.726 e-10 \quad 1.885 e-10 \quad 2.155 e-10$ 1.110e-02 1.218e-10 $1.408 e-10 \quad 1.550 e-10 \quad 1.690 e-10 \quad 1.846 e-10 \quad 2.110 e-10$ 1.190e-02 $1.166 e-10 \quad 1.349 e-10 \quad 1.486 e-10 \quad 1.621 e-10 \quad 1.769 e-10 \quad 2.025 e-10$ 1.270e-02 $1.073 e-10 \quad 1.242 e-10 \quad 1.370 e-10 \quad 1.495 e-10 \quad 1 \quad 32 e-10 \quad 1.869 e-10$ 1.300e-02 9.963e-11 $1.155 e-10 \quad 1.274 e-10 \quad 1.392 e-10 \quad 1.519 e-10 \quad 1.741 e-10$ $\begin{array}{lllllll}1.390 e-02 & 0.987 e-11 & 1.043 e-10 & 1.152 e-10 & 1.260 e-10 & 1.374 e-10 & 1.578 e-10\end{array}$ 1.470e-02 7.403e-11 8.610e-11 $9.544 e-11 \quad 1.046 e-10 \quad 1.139 e-10 \quad 1.312 e-10$ 1.520e-02 6.102e-11 $7.119 e-11 \quad 7.916 e-11 \quad 8.702 e-11 \quad 9.458 e-11 \quad 1.093 e-10$ 1.570e-02 5.773e-11 6.742e-11 7.501e-11 8.254e-11 8.969e-11 $1.038 e-10$ $1.640 e-32$ 5.558e-11 $6.496 e-11 \quad 7.227 e-11 \quad 7.958 e-11 \quad 8.648 e-11 \quad 1.001 e-10$ $1.700 e-025.289 e-11 \quad 6.189 e-11 \quad 6.884 e-11 \quad 7.588 e-11 \quad 8.246 e-11 \quad 9.552 e-11$ 1.720e-02 5.105e-11 5.979e-11 6.650e-11 7.335e-11 7.971e-11 9.237e-11 $\begin{array}{llllllll}1.810 e-02 & 4.824 e-11 & 5.657 e-11 & 6.291 e-11 & 6.946 e-11 & 7.550 e-11 & 8.755 e-11\end{array}$ $\begin{array}{lllllll}1.900 e-02 & 4.310 e-11 & 5.067 e-11 & 5.633 e-11 & 6.234 e-11 & 6.776 e-11 & 7.869 e-11\end{array}$ $1.970 e-02 \quad 3.792 e-11 \quad 4.472 e-11 \quad 4.970 e-11 \quad 5.516 e-11 \quad 5.996 e-11 \quad 6.975 e-11$ 2.010e-02 3.411e-11 $4.035 e-11 \quad 4.482 e-11 \quad 4.988 e-11 \quad 5.422 e-11 \quad 6.316 e-11$ 2.100e-02 3.286e-11 $3.891 e-11 \quad 4.322 e-11 \quad 4.814 e-11 \quad 5.234 e-11 \quad 6.100 e-11$ 2.170e-02 3.212e-11 3.806e-11 $4.226 e-11 \quad 4.710 e-11 \quad 5.122 e-11 \quad 5.971 e-11$ 2.230e-02 3.141e-11 3.724e-11 4.134e-11 4.610e-11 $5.014 e-11 \quad 5.846 e-11$ 2.310e-02 3.053e-11 3.622e-11 4.n19e-11 4.485e-11 4.879e-11 $5.690 e-11$ 2.550a-02 2.803e-11 3.333e-11 3.695e-11 4.130e-11 4.495e-11 5.247e-11 2.920e-02 2.161e-11 2.587e-11 2.859e-11 $3.214 e-11 \quad 3.503 e-11 \quad 4.099 e-11$ $3.320 e-02 \quad 1.414 e-11 \quad 1.717 e-11 \quad 1.885 e-11 \quad 2.144 e-11 \quad 2.344 e-11 \quad 2.756 e-11$ $3.750 e-02 \quad 1.162 e-11 \quad 1.419 e-11 \quad 1.553 e-11 \quad 1.775 e-11 \quad 1.944 e-11 \quad 2.289 e-11$ 4.200e-02 $8.454 e-12 \quad 1.043 e-12 \quad 1.133 e-11 \quad 1.307 e-11 \quad 1.436 e-11 \quad 1.695 e-11$ 4.740e-02 6.775e-12 $8.420 e-12 \quad 9.096 e-12 \quad 1.055 e-11 \quad 1.162 e-11 \quad 1.374 e-11$ 4.850e-02 5.762e-12 $7.195 e-12 \quad 7.740 e-12 \quad 9.013 e-12 \quad 9.945 e-12 \quad 1.177 e-11$

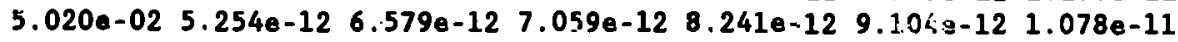
5.500e-02 4.678e-12 5.876e-12 6.282e-12 $7.356 e-12 \quad 8.136 e-12 \quad 9.637 e-12$ 6.100e-02 3.845e-12 4.851e-12 $5.156 e-12 \quad 6.062 e-12 \quad 6.718 e-12 \quad 7.959 e-12$ 6.750e-02 3.267e-12 $4.134 e-12 \quad 4.370 e-12 \quad 5.154 e-12 \quad 5.719 e-12 \quad 6.775 e-12$ 6.950e-02 3.016e-12 $3.818 e-12 \quad 4.026 e-12 \quad 4.751 e-12 \quad 5.275 e-12 \quad 6.847 e-12$ $\begin{array}{lllllll}7.350 e-02 & 2.798 e-12 & 3.543 e-12 & 3.727 e-12 & 4.403 e-12 & 4.889 e-12 & 5.789 e-12\end{array}$ 7. 840e-02 2. $422 e-12 \quad 3.070 e-12 \quad 3.213 e-12 \quad 3.801 e-12 \quad 4.225 e-12 \quad 4.999 e-12$

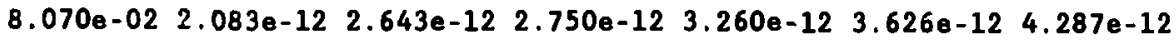
8.550e-02 1.962e-12 2.488e-12 2.581e-12 $3.060 e-12 \quad 3.403 e-12 \quad 4.021 e-12$ 8.800e-02 $1.873 e-12 \quad 2.371 e-12 \quad 2.455 e-12 \quad 2.908 e-12 \quad 3.234 e-12 \quad 3.818 e-12$

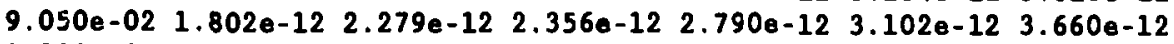
$9.230 e-02 \quad 1.736 e-12 \quad 2.193 e-12 \quad 2.263 e-12 \quad 2.679 e-12 \quad 2.978 e-12 \quad 3.512 e-12$ 


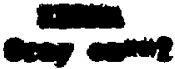

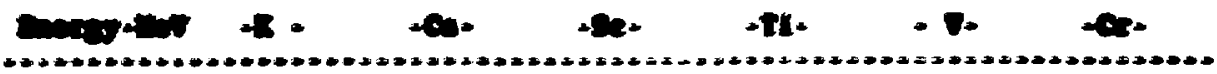

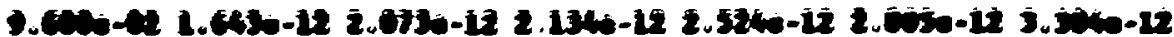
1.cmen 1.

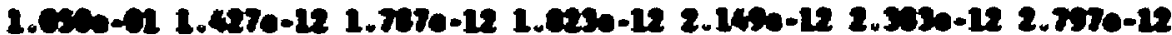

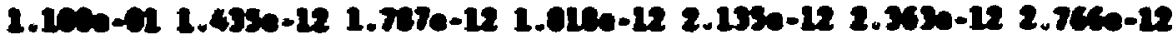

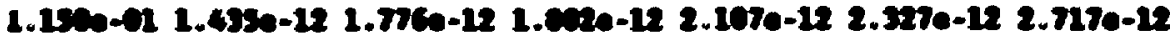
1.210-01 1.425-12 1.7520-12 $1.7710-12$ 2.6510-12 $2.265-12$ 2.c40-12

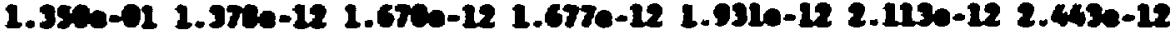

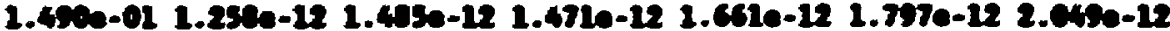

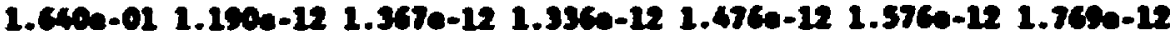

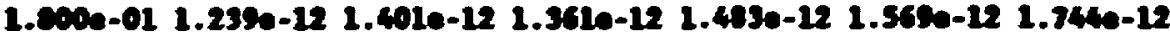
1.900-01 $1.2660-22$ 1.605e-12 $1.3550-12$ 1.450e-12 $1.517 e-12$ 1.65se-12 2.190-01 $1.3140-22$ 1.434e-12 $1.373 e-12$ 1.446-12 $1.4360-12$ 1.620e-12 2.390e-01 $1.415 e-12 \quad 1.531 e-12 \quad 1.463 e-12 \quad 1.527 e-12 \quad 1.570 e-12 \quad 1.690 e-12$ 2.630e-01 $1.501 e-12 \quad 1.616 e-12 \quad 1.540 e-12 \quad 1.5930-12 \quad 1.627 e-12 \quad 1.734 e-12$ 2.300e-01 $1.591 e-121.689 e-12 \quad 1.602 e-12 \quad 1.6380-12 \quad 1.659 e-12 \quad 1.755 e-12$ 3.150e-01 $1.673 e-12 \quad 1.757 e-12 \quad 1.661 e-12 \quad 1.676 e-12 \quad 1.684 e-12 \quad 1.765 e-12$ 3.510e-01 $1.799 e-12 \quad 1.892 e-12 \quad 1.776 e-12 \quad 1.706 e-12 \quad 1.786 e-12 \quad 1.864 e-12$ 3.860e-01 $1.924 e-12$ 2.005e-12 $1.089 e-12$ 1.891e-12 $1.844 e-12$ 1.95ee-12 4.240e-01 2.047e-12 $2.124 e-12$ 1.999e-12 $1.991 e-12$ 1.976e-12 $2.045 e-12$ 4.670e-01 2.194e-12 2.272e-12 $2.137 e-12$ 2.123e-12 $2.103 e-12 \quad 2.172 e-12$ 5.130e-01 $2.342 e-12 \quad 2.420 e-12 \quad 2.274 e-12 \quad 2.254 e-12 \quad 2.228 e-12 \quad 2.295 e-12$ 5.650e-01 2.571e-12 $2.652 e-12 \quad 2.491 e-12 \quad 2.464 e-12 \quad 2.432 e-12 \quad 2.502 e-12$ 6.210e-01 2.828e-12 2.914e-12 2.735e-12 2.702e-12 2.664e-12 2.736e-12 6.830e-01 3.105e-12 $3.197 e-12 \quad 3.000 e-12 \quad 2.960 e-12 \quad 2.918 e-12 \quad 2.994 e-12$ 7.510e-01 $3.384 e-12 \quad 3.482 e-12 \quad 3.266 e-12 \quad 3.221 e-12 \quad 3.173 e-12 \quad 3.253 e-12$ 8.260e-01 3.654e-12 $3.757 e-12 \quad 3.523 e-12 \quad 3.471 e-12 \quad 3.417 e-12 \quad 3.501 e-12$ 9.090e-01 3.950e-12 $4.060 e-12 \quad 3.807 e-12 \quad 3.743 e-12 \quad 3.690 e-12 \quad 3.779 e-12$ $1.000 e+00 \quad 4.245 e-12 \quad 4.362 e-12 \quad 4.089 e-12 \quad 4.025 e-12 \quad 3.961 e-12 \quad 4.055 e-12$ $1.120 e+00 \quad 4.644 e-12 \quad 4.771 e-12 \quad 4.471 e-12 \quad 4.400 e-12 \quad 4.329 e-12 \quad 4.431 e-12$ $1.270 e+00 \quad 5.175 e-12 \quad 5.316 e-12 \quad 4.982 e-12 \quad 4.902 e-12 \quad 4.823 e-12 \quad 4.935 e-12$ $1.420 e+00 \quad 5.671 e-12 \quad 5.826 e-12 \quad 5.459 e-12 \quad 5.371 e-12 \quad 5.284 e-12 \quad 5.407 e-12$ $\begin{array}{lllllll}1.600 e+00 & 6.132 e-12 & 6.300 e-12 & 5.902 e-12 & 5.808 e-12 & 5.713 e-12 & 5.846 e-12\end{array}$ $1.800 e+00 \quad 6.706 e-12 \quad 6.892 e-1.26 .459 e-12 \quad 6.357 e-12 \quad 6.255 e-12 \quad 6.401 e-12$ $2.030 e+007.273 e-12 \quad 7.477 e-12 \quad 7.010 e-12 \quad 6.902 e-12 \quad 6.794 e-12 \quad 6.956 e-12$ $2.280 e+00$ 8.001e-12 8.231e-12 7.721e-12 7.607e-12 $7.492 e-12 \quad 7.675 e-12$ $2.570 e+00 \quad 8.862 e-12 \quad 9.124 e-12 \quad 8.565 e-12 \quad 8.445 e-12 \quad 8.324 e-12 \quad 8.535 e-12$ 2.890e+00 9.711e-12 $1.001 e-11 \quad 9.406 e-12 \quad 9.284 e-12 \quad 9.161 e-12 \quad 9.402 e-12$ $3.250 e+001.059 e-11 \quad 1.093 e-11 \quad 1.029 e-11 \quad 1.017 e-11 \quad 1.004 e-11 \quad 1.032 e-11$ $3.650 e+001.171 e-11 \quad 1.210 e-11 \quad 1.141 e-11 \quad 1.129 e-11 \quad 1.117 e-11 \quad 1.149 e-11$ 4.110e+00 1.289e-11 $1.334 e-11$ 1.260e-11 $1.249 e-11 \quad 1.238 e-11 \quad 1.276 e-11$ $4.620 e+001.435 e-11 \quad 1.499=-11 \quad 1.407 e-11 \quad 1.398 e-11 \quad 1.387 e-11 \quad 1.432 e-11$ $5.200 e+001.600 e-11 \quad 1.664 e-11 \quad 1.576 e-11 \quad 1.568 e-11 \quad 1.559 e-11 \quad 1.612 e-11$ $5.850 e+001.785 e-11 \quad 1.860 e-11 \quad 1.765 e-11 \quad 1.759 e-11 \quad 1.752 e-11 \quad 1.815 e-11$ $6.580 e+001.999 e-11 \quad 2.087 e-11 \quad 1.985 e-11 \quad 1.982 e-11 \quad 1.978 e-11 \quad 2.052 e-11$ $7.410 e+002.265 e-112.369 e-11 \quad 2.257 e-11 \quad 2.258 e-11 \quad 2.258 e-11 \quad 2.348 e-11$ 


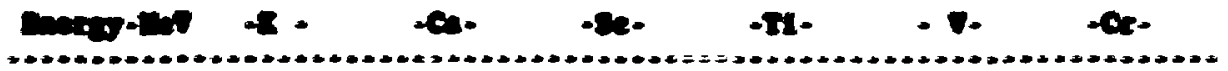

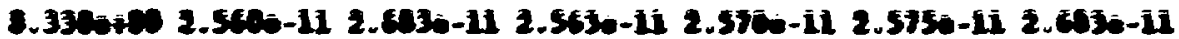

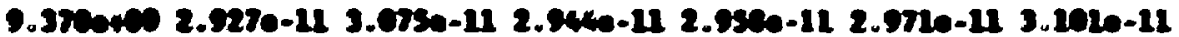

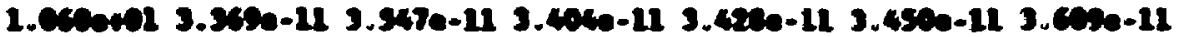

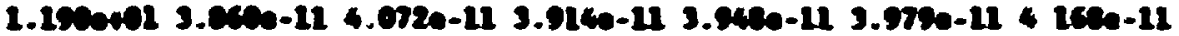

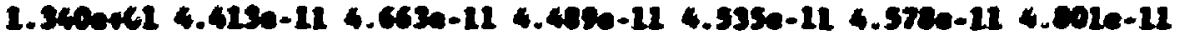

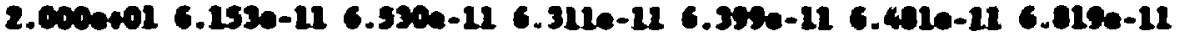

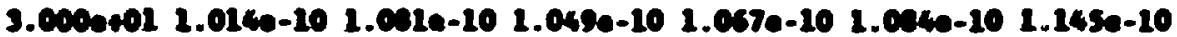
4.0000401 1.5240-10 $1.6340-101.5090-101.6200-101.690 e-101.7440-10$ 5.000e+01 2.600-10 2.226-10 2.167e-10 2.213e-10 2.255e-10 2.377-10 7.5006+01 3.037e-10 3.304-10 3.225e-10 3.2570-10 3.364e-10 3.546e-10 1.000e+e2 4.614e-10 4.947a-10 4.027e-10 4.934e-10 5.043e-10 5.346e-10 


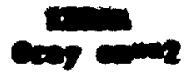

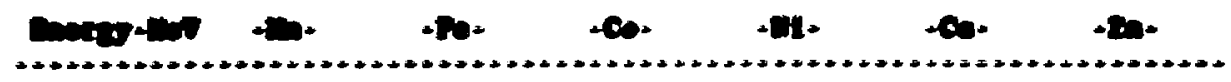
$1 . \cos 0.4$

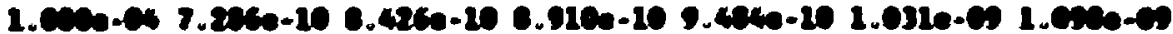

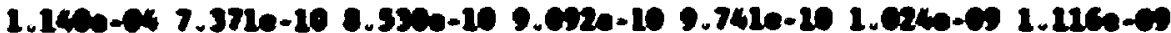

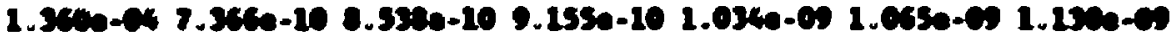

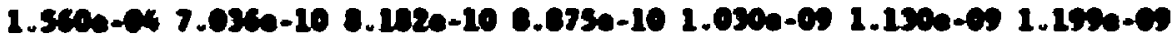

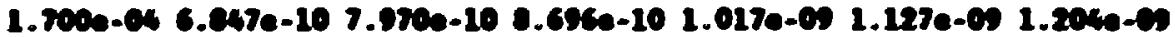

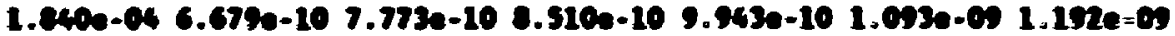

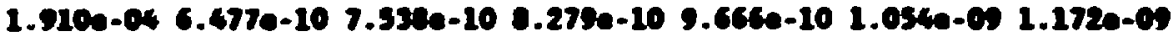 2.140e-04 6.105e-10 7.204e-10 7.947e-10 9.271e-10 $1.002 e-09$ 1.140e-6s $2.240-046.1010-107.1040-107.0420-10 \quad 9.152 e-109.879 e-10$ 1.130e-es 2.410e-04 6.014e-10 $6.992 e-107.721 e-10 \quad 9.0160-10 \quad 9.7360-10 \quad 1.1260-09$ $2.670 e-045.896 e-10 \quad 6.652 e-107.570 e-10 \quad 8.845 e-10 \quad 9.554 a-10 \quad 1.057 a-05$ 2.630e-04 5.740e-10 $6.668 e-107.370 e-10 \quad e .617 e-10 \quad 9.312 e-10 \quad 1.0720-05$ 2.750e-04 $5.495 e-10 \quad 6.379 e-10 \quad 7.056 e-10 \quad 8.257 e-10 \quad s .928 e-10 \quad 1.031 e-05$ 2.820e-04 $5.295 e-10 \quad 5.1440-10 \quad 6.800 e-10 \quad 7.964 e-10 \quad 0.615 e-10 \quad 9.973 e-10$ $2.960 e-045.040 e-10 \quad 5.844 e-10 \quad 6.472 e-10 \quad 7.584 e-10 \quad 8.213 e-10 \quad 9.540 e-10$ 3.180e-04 4.719e-10 $5.468 e-10 \quad 6.061 e-10 \quad 7.113 e-10 \quad 7.704 e-10 \quad 8.986 e-10$ 3.550e-04 4.515e-10 5.226e-10 5.794e-10 $6.796 e-10 \quad 7.360 e-10 \quad$ e.593e-10 $3.610 e-044.313 e-10 \quad 4.992 e-10 \quad 5.530 e-10 \quad 6.493 e-10 \quad 7.021 e-10 \quad 8.203 e-10$ $3.700 e-04 \quad 4.225 e-10 \quad 4.889 e-10 \quad 5.416 e-10 \quad 6.348 e-10 \quad 6.874 e-10 \quad s .035 e-10$ $3.830 e-04 \quad 4.083 e-10 \quad 4.724 e-10 \quad 5.232 e-10 \quad 6.130 e-10 \quad 6.638 e-10 \quad 7.762 e-10$ $4.030 e-04 \quad 3.844 e-10 \quad 4.445 e-10 \quad 4.921 e-10 \quad 5.762 e-10 \quad 6.239 e-10 \quad 7.302 e-10$ $4.150 e-04 \quad 3.692 e-10 \quad 4.268 e-10 \quad 4.723 e-10 \quad 5.533 e-10 \quad 6.009 e-10 \quad 7.012 e-10$ $4.300 e-04 \quad 3.625 e-10 \quad 4.191 e-10 \quad 4.635 e-10 \quad 5.438 e-10 \quad 5.935 e-10 \quad 6.886 e-10$ $4.500 e-04 \quad 3.521 e-10 \quad 4.070 e-10 \quad 4.498 e-10 \quad 5.288 e-10 \quad 5.813 e-10 \quad 6.688 e-10$ $4.810 e-04 \quad 3.332 e-10 \quad 3.851 e-10 \quad 4.249 e-10 \quad 5.015 e-10 \quad 5.582 e-10 \quad 6.330 e-10$ 5.160e-04 3.050e-10 $3.524 e-10 \quad 3.881 e-10 \quad 4.607 e-10 \quad 5.217 e-10 \quad 5.795 e-10$ $5.470 e-04 \quad 2.877 e-10 \quad 3.319 e-10 \quad 3.665 e-10 \quad 4.361 e-10 \quad 4.926 e-10 \quad 5.448 e-10$ 5.550e-04 2.776e-10 3.199e-10 3.541e-10 4.217e-10 4.741e-10 5.244e-10 5.930e-04 2.635e-10 $3.032 e-10 \quad 3.366 e-10 \quad 4.015 e-10 \quad 4.482 e-10 \quad 4.958 e-10$ $5.980 e-04 \quad 2.500 e-10 \quad 2.875 e-10 \quad 3.185 e-10 \quad 3.805 e-10 \quad 4.215 e-10 \quad 4.662 e-10$ 6.250e-04 2.432e-10 2.805e-10 $3.101 e-10 \quad 3.695 e-10 \quad 4.067 e-10 \quad 4.528 e-10$ 6.540e-04 5.651e-10 2.682e-10 2.981e-10 3.525e-10 3.831e-10 4.341e-10 6.750e-04 1.653e-09 2.555e-10 2.856e-10 3.350e-10 3.591e-10 4.146e-10 6.980e-04 1.793e-09 2.454e-10 2.731e-10 3.175e-10 $3.354 e-10 \quad 3.951 e-10$ 7.110e-04 1.734e-09 2.382e-10 2.633e-10 3.048e-10 3.198e-10 3.804e-10 7.250e-04 1.695e-09 1.033e-09 2.588e-10 3.007e-10 3.185e-10 3.745e-10 7.710e-04 1.613e-09 $1.638 e-09 \quad 2.473 e-10 \quad 2.902 e-10 \quad 3.150 e-10 \quad 3.594 e-10$

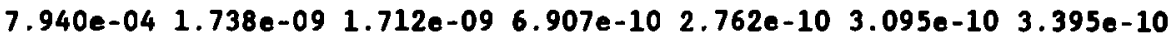 8.180e-04 1.662e-09 $1.643 \mathrm{e}-09 \quad 1.684 \mathrm{e}-09 \quad 2.654 \mathrm{e}-10 \quad 3.019 \mathrm{e}-10 \quad 3.258 \mathrm{e}-10$ 8.380e-04 1.618e-09 1.584e-09 1.684e-09 2.546e-10 2.875e-10 3.157e-10 8.600e-04 1.573e-09 1.686e-09 1.655e-09 2.443e-10 2.724e-10 3.053e-10 8.980e-04 1.501e-09 1.696e-09 1.587e-09 1.262e-09 2.487e-10 2.887e-10 $9.200 e-04 \quad 1.442 e-09 \quad 1.623 e-09 \quad 1.509 e-09 \quad 1.753 e-092.313 e-10 \quad 2.734 e-10$ $9.400 e-041.432 e-091.597 e-091.647 e-09 \quad 1.741 e-09 \quad 3.225 e-10 \quad 2.662 e-10$ $9.560 \mathrm{e}-041.421 \mathrm{e}-091.574 \mathrm{e}-09 \quad 1.720 \mathrm{e}-09 \quad 1.728 \mathrm{e}-09 \quad 1.318 \mathrm{e}-092.596 \mathrm{e}-10$ 

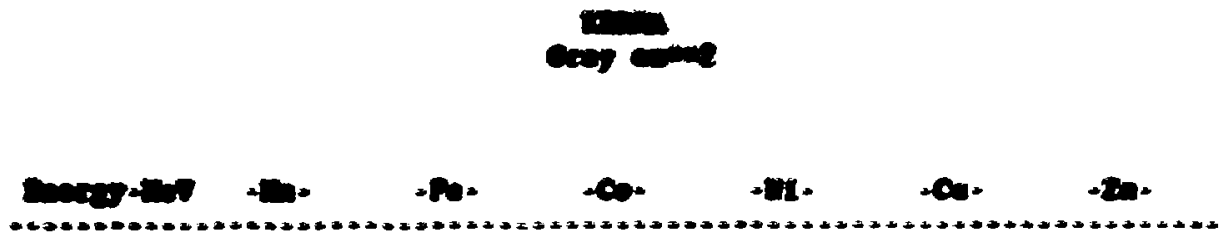

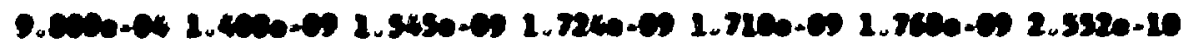

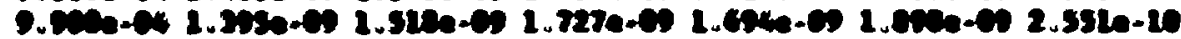

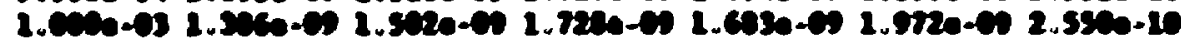

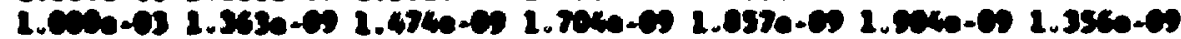

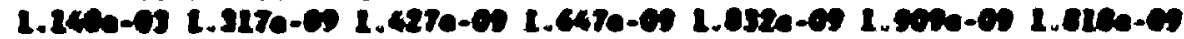

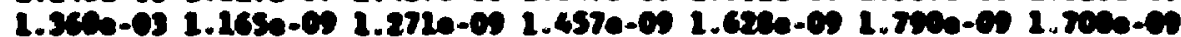

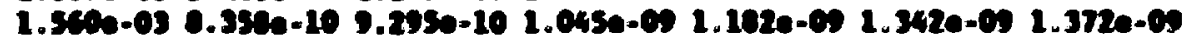

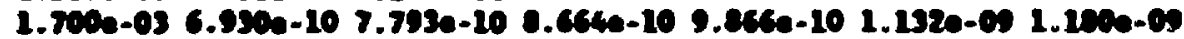

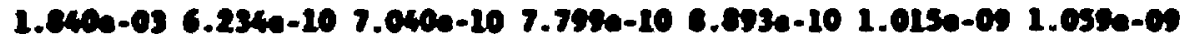

1.910e-03 $5.5330-10 \quad 6.3350-10 \quad 6.9310-107.9440-10 \quad 9.0940-10 \quad 9.457 e-10$

2.140e-03 $4.7520-10 \quad 5.431 e-10 \quad 5.952 e-10 \quad 6.0210-10 \quad 7.676-10 \quad 0.018-10$

2.240e-03 4.452e-10 $5.101 e-105.5060-10 \quad 6.401 e-10 \quad 7.1030-10 \quad 7.502 e-10$

2.410e-03 4.2390-10 4.064e-10 $5.3230-10 \quad 6.103 e-10 \quad 6.036 e-10 \quad 7.153 e-10$

2.470e-03 4.019a-10 4.616a-10 5.050e-10 5.754a-10 6.476e-10 $6.744 e-10$

2.630e-03 3.764e-10 4.332e-10 $4.733 e-10 \quad 5.436 e-10 \quad 6.0530-10 \quad 6.356 e-10$

2.750e-03 $3.3910-10 \quad 3.913 e-10 \quad 4.270 e-10 \quad 4.9100-10 \quad 5.457 e-10 \quad 5.733 e-10$

2.820e-03 3.103e-10 $3.590 e-10 \quad 3.913 e-10 \quad 4.5040-10 \quad 4.963 e-10 \quad 5.251 e-10$

2.960e-03 $2.749 e-10 \quad 3.193 e-10 \quad 3.474 e-10 \quad 4.005 e-10 \quad 4.415 e-10 \quad 4.655 e-10$

3.100-03 $2.320 e-10 \quad 2.709 e-10 \quad 2.940 e-10 \quad 3.398 e-10 \quad 3.719 e-10 \quad 3.941 e-10$

3.550--03 2.101e-10 2.457e-10 2.668e-10 3.088e-10 $3.369 e-10 \quad 3.582 e-10$

$3.610 e-03 \quad 1.910 e-10 \quad 2.237 e-10 \quad 2.430 e-10 \quad 2.816 e-10 \quad 3.064 e-10 \quad 3.269 e-10$

3.700e-03 $1.833 e-10 \quad 2.147 e-10 \quad 2.334 e-10 \quad 2.705 e-10 \quad 2.940 e-10 \quad 3.141 e-10$

3.830e-03 $1.710 a-10 \quad 2.006 e-10 \quad 2.180 e-10 \quad 2.530 a-10 \quad 2.744 e-10 \quad 2.938 e-10$

4.030e-03 $1.508 e-10 \quad 1.773 e-10 \quad 1.929 e-10 \quad 2.241 e-10 \quad 2.4 \varepsilon=e-10 \quad 2.606 e-10$

$4.150 e-03 \quad 1.385 e-10 \quad 1.630 e-10 \quad 1.775 e-10 \quad 2.065 e-10 \quad 2.226 e-10 \quad 2.403 e-10$

$4.300 e-03 \quad 1.336 e-10 \quad 1.573 e-10 \quad 1.714 e-10 \quad 1.995 e-10 \quad 2.149 e-10 \quad 2.324 e-10$

$4.500 e-03 \quad 1.265 e-10 \quad 1.489 e-10 \quad 1.625 e-10 \quad 1.892 e-10 \quad 2.035 e-10 \quad 2.206 e-10$

4.810e-03 $1.142 e-10 \quad 1.345 e-10 \quad 1.470 e-10 \quad 1.714 e-10 \quad 1.840 e-10 \quad 2.003 e-10$

5.160e-03 $9.674 e-11 \quad 1.141 e-10 \quad 1.252 e-10 \quad 1.462 e-10 \quad 1.564 e-10 \quad 1.716 e-10$

5.470e-03 $8.737 e-11 \quad 1.031 e-10 \quad 1.134 e-10 \quad 1.325 e-10 \quad 1.415 e-10 \quad 1.561 e-10$

5.550e-03 $8.240 e-11 \quad 9.719 e-11 \quad 1.071 e-10 \quad 1.253 e-10 \quad 1.337 e-10 \quad 1.479 e-10$

5.930e-03 7.565e-11 8.921e-11 9.856e-11 $1.154 e-10 \quad 1.230 e-10 \quad 1.367 e-10$

$5.980 e-03 \quad 6.877 e-11 \quad 8.108 e-11 \quad 8.985 e-11 \quad 1.053 e-10 \quad 1.120 e-10 \quad 1.252 e-10$

6.250e-03 6.529e-11 7.751e-11 8.651e-11 $1.016 e-10 \quad 1.081 e-10 \quad 1.211 e-10$

$6.540 e-03 \quad 6.082 e-11 \quad 7.273 e-11 \quad 8.262 e-11 \quad 9.772 e-11 \quad 1.039 e-10 \quad 1.166 e-10$

6.750e-03 4.849e-10 $6.789 e-11 \quad 7.861 e-11 \quad 9.363 e-11 \quad 9.947 e-11 \quad 1.120 e-10$

6.980e-03 4.687e-10 6.314e-11 $7.462 e-11 \quad 8.951 e-11 \quad 9.505 e-11 \quad 1.073 e-10$

7.110e-03 4.539e-10 5.893e-11 7.106e-11 8.582e-11 9.109e-11 1.031e-10

$7.250 e-03 \quad 4.419 e-10 \quad 4.616 e-10 \quad 6.818 e-11 \quad 8.284 e-11 \quad 8.788 e-11 \quad 9.967 e-11$

7.710e-03 4.121e-10 4.421e-10 6.250e-11 7.550a-11 8.002e-1: 9.128e-11

$7.940 e-03 \quad 3.737 e-10 \quad 4.108 e-10 \quad 4.539 e-10 \quad 6.616 e-11 \quad 7.000 e-11 \quad 8.056 e-11$

8.180e-03 3.506e-10 $3.914 e-10 \quad 4.353 e-10 \quad 6.034 e-11 \quad 6.395 e-11 \quad 7.426 e-11$

8.380e-03 3.413e-10 3.818e-10 4.246e-10 $1.491 e-10 \quad 6.130 e-11 \quad 7.196 e-11$

8.600e-03 $3.321 e-10 \quad 3.722 e-10 \quad 4.136 e-10 \quad 4.366 e-10 \quad 5.871 e-11 \quad 6.974 e-11$

8.980e-03 $3.173 e-10 \quad 3.567 e-10 \quad 3.959 e-10 \quad 4.206 e-10 \quad 5.607 e-11 \quad 6.621 e-11$

Page -22- 


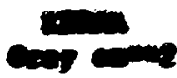

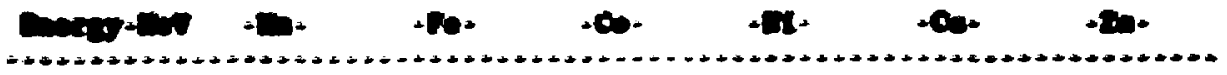

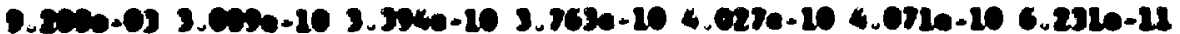

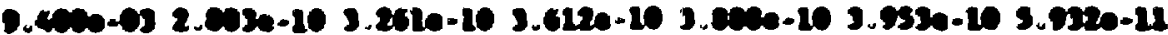

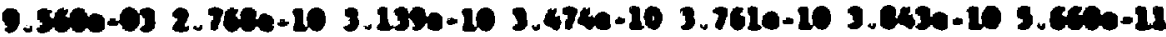

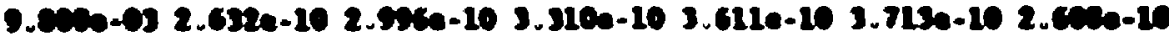

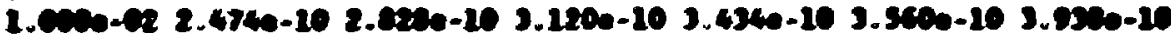

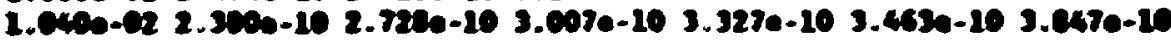

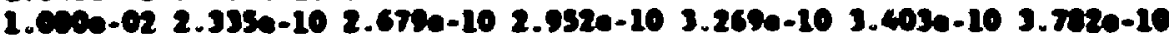

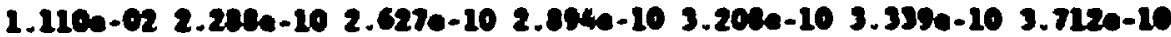

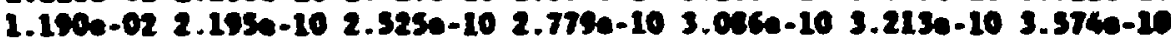
$\begin{array}{lllllll}1.270 e-02 & 2.027 e-10 & 2.334--10 & 2.372 e-10 & 2.065 e-10 & 2.303 e-10 & 3.3220-10\end{array}$ 1.300e-02 $1.035 e-102.185 e-102.401 e-10 \quad 2.682 e-10 \quad 2.753 e-10 \quad 3.113 e-10$

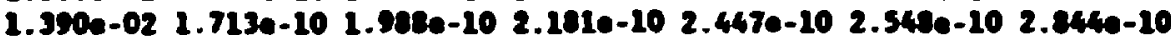
1.470e-02 $1.426 e-101.667 e-101.024 e-10$ 2.063e-10 2.150e-10 2.406e-10 1.520e-02 $1.190 a-10 \quad 1.4030-10 \quad 1.531 e-10 \quad 1.747 e-10 \quad 1.821 e-10 \quad 2.045 a-10$ $1.570 e-02 \quad 1.130 e-10 \quad 1.335 e-10 \quad 1.455 e-10 \quad 1.655 e-10 \quad 1.736 e-10 \quad 1.950 e-10$ 1.640e-02 $1.090 e-101.269 e-10 \quad 1.404 e-10 \quad 1.608 e-10 \quad 1.677 e-10 \quad 1.295 e-10$ 1.700e-02 $1.040 e-10 \quad 1.231 e-10 \quad 1.341 e-10 \quad 1.537 e-10 \quad 1.604 e-10 \quad 1.803 e-10$ 1.720e-02 $1.006 e-10 \quad 1.191 e-10 \quad 1.297 e-10 \quad 1.489 e-10 \quad 1.554 e-10 \quad 1.747 e-10$ $1.810 e-02 \quad 9.539 e-11 \quad 1.130 e-10 \quad 1.230 e-10 \quad 1.414 e-10 \quad 1.477 e-10 \quad 1.661 e-10$ $1.900 e-02 \quad 8.579 e-11 \quad 1.019 e-10 \quad 1.108 e-10 \quad 1.277 e-10 \quad 1.334 e-10 \quad 1.502 e-10$ $1.970 e-02 \quad 7.610 e-11 \quad 9.055 e-11 \quad 9.841 e-11 \quad 1.138 e-10 \quad 1.191 e-10 \quad 1.341 e-10$ 2.010e-02 $6.897 e-11 \quad 8.222 e-11 \quad 8.928 e-11 \quad 1.036 e-10 \quad 1.055 e-10 \quad 1.223 e-10$ $2.100 e-02 \quad 6.663 e-11 \quad 7.947 e-11 \quad 8.628 e-11 \quad 1.002 e-10 \quad 1.050 e-10 \quad 1.183 e-10$ 2.170e-02 $6.523 e-11 \quad 7.781 e-11 \quad 8.446 e-11 \quad 9.809 e-11 \quad 1.028 e-10 \quad 1.159 e-10$ 2.230e-02 6.387e-11 $7.619 e-11 \quad 8.270 e-11 \quad 9.608 e-11 \quad 1.008 e-10 \quad 1.136 e-10$ 2.310e-02 6.217e-11 7.417e-11 8.050e-11 9.356e-11 9.816e-11 $1.106 e-10$ 2.550e-02 5.734e-11 $6.843 e-11 \quad 7.425 e-11 \quad 8.639 e-11 \quad 9.072 e-11 \quad 1.023 e-10$ 2.920e-02 4.486e-11 $5.356 e-11 \quad 5.808 e-11 \quad 6.780 e-11 \quad 7.242 e-11 \quad 8.052 e-11$ 3.320e-02 3.023e-11 $3.613 e-11 \quad 3.913 e-11 \quad 4.596 e-11 \quad 4.872 e-11 \quad 5.494 e-11$ 3.750e-02 2.513e-11 2.999e-11 $3.249 e-11 \quad 3.820 e-11 \quad 4.062 e-11 \quad 4.576 e-11$ 4.200e-02 1.864e-11 2.217e-11 2.403e-11 2.829e-11 $3.025 e-11 \quad 3.402 e-11$ 4.740e-02 $1.512 e-11 \quad 1.793 e-11 \quad 1.944 e-11 \quad 2.289 e-11 \quad 2.457 e-11 \quad 2.760 e-11$ 4.850e-02 1.296e-11 $1.532 a-11 \quad 1.663 e-11 \quad 1.958 a-11 \quad 2.106 e-11 \quad 2.364 e-11$ 5.020e-02 1.187e-11 $1.401 e-11 \quad 1.521 e-11 \quad 1.791 e-11 \quad 1.930 e-11 \quad 2.165 e-11$ 5.500e-02 $1.062 e-11 \quad 1.250 a-11 \quad 1.358 a-11 \quad 1.598 e-11 \quad 1.726 e-11 \quad 1.934 a-11$ 6.100e-02 8.775e-12 $1.029 e-11 \quad 1.118 e-11 \quad 1.315 e-11 \quad 1.424 e-11 \quad 1.593 e-11$ $\begin{array}{llllllll}6.750 e-02 & 7.473 e-12 & 8.725 e-12 & 9.489 e-12 & 1.114 e-11 & 1.210 e-11 & 1.352 e-11\end{array}$ 6.950e-02 6.890e-12 8.026e-12 8.733e-12 $1.024 e-11 \quad 1 \quad 114 a-11 \quad 1.243 e-11$

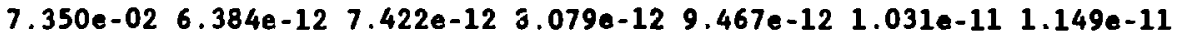

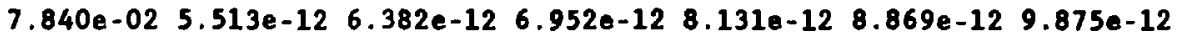

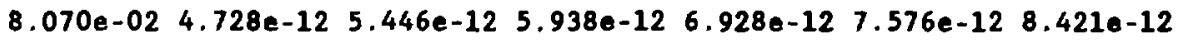
$\begin{array}{lllllll}8.550 e-02 & 4.433 e-12 & 5.095 e-12 & 5.557 e-12 & 6.476 e-12 & 7.086 e-12 & 7.870 e-12\end{array}$ $8.800 e-02 \quad 4.208 e-12 \quad 4.828 e-12 \quad 5.265 e-12 \quad 6.130 e-12 \quad 6.710 e-12 \quad 7.447 e-12$ 9.050e-02 4.032e-12 $4.619 e-12 \quad 5.037 e-12 \quad 5.860 e-12 \quad 6.416 e-12 \quad 7.117 e-12$ $9.230 e-02 \quad 3.868 e-12 \quad 4.424 e-12 \quad 4.825 e-12 \quad 5.609 e-12 \quad 6.143 e-12 \quad 6.820 e-12$ 


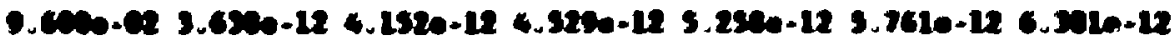

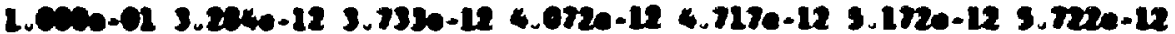

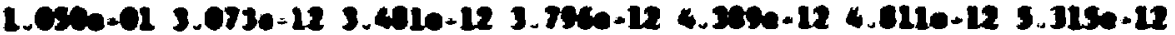

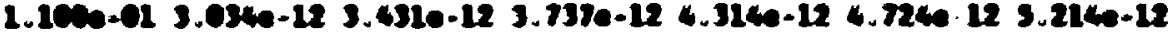

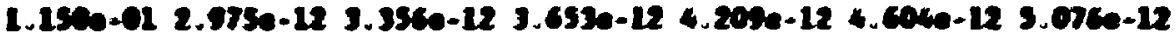

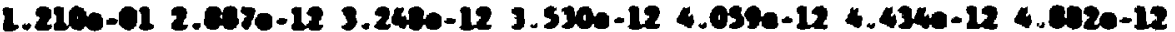
1.350e-01 2.65se-12 2.972e-12 $3.222 e-12 \quad 3.69 e-12 \quad 4.016-12 \quad 4.406-12$ $1.450-012.2120-122.4390-122.6290-12 \quad 2.9790-12 \quad 3.223-12 \quad 3.5150-12$ 1.660-01 $1.0050-122.0540-122.1970-12 \quad 2.4600-12 \quad 2.634-122.0530-12$ 1.000-02 $1.040-12$ 1.996e-12 $2.122 e-12$ 2.361e-12 $2.512 e-12$ 2.703e-12 1.900-01 $1.746 e-12 \quad 1.868 e-12 \quad 1.9700-12 \quad 2.170-12 \quad 2.246 e-12 \quad 2.4400-12$ 2.1000-01 $1.6030-12 \quad 1.7050-12 \quad 1.0650-12 \quad 2.036 e-12 \quad 2.117 e-12 \quad 2.244 e-12$ 2.390e-01 1.747e-12 1.845e-12 $1.917 e-12$ 2.094e-12 $2.151 e-12 \quad 2.271 e-12$ 2.630e-01 $1.706 a-12 \quad 1.877 a-12 \quad 1.9390-12 \quad 2.0950-12 \quad 2.143 e-12 \quad 2.252 e-12$ 2.500e-01 $1.789 e-12 \quad 1.0700-12 \quad 1.9150-12 \quad 2.0540-12 \quad 2.0760-12 \quad 2.166 e-12$ 3.190e-01 $1.703 e-12 \quad 1.052 e-12 \quad 1.879 e-12 \quad 1.999 e-12 \quad 1.993 e-12 \quad 2.062 e-12$ 3.510e-01 $1.076 e-12 \quad 1.943 e-12 \quad 1.963 e-12 \quad 2.091 e-12 \quad 2.062 e-12 \quad 2.126 e-12$ $\begin{array}{llllllll}3.060 e-01 & 1.963 e-12 & 2.029 e-12 & 2.040 e-12 & 2.156 e-12 & 2.122 e-12 & 2.179 e-12\end{array}$ $\begin{array}{llllllll}4.240 e-01 & 2.043 e-12 & 2.106 e-12 & 2.109 e-12 & 2.220 a-12 & 2.170 e-12 & 2.219 e-12\end{array}$ $\begin{array}{lllllll}4.670 e-01 & 2.164 e-12 & 2.229 e-12 & 2.225 e-12 & 2.338 e-12 & 2.276 e-12 & 2.322 e-12\end{array}$ $\begin{array}{lllllll}5.130 e-01 & 2.282 e-12 & 2.347 e-12 & 2.337 e-12 & 2.451 e-12 & 2.377 e-12 & 2.418 e-12\end{array}$

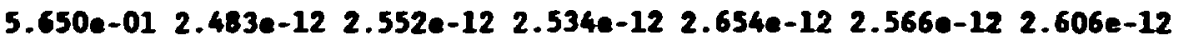
6.210e-01 $2.712 e-12 \quad 2.784 e-12 \quad 2.760 e-12 \quad 2.887 e-12 \quad 2.783 e-12 \quad 2.821 e-12$ 6.830e-01 $2.965 e-12 \quad 3.042 e-12 \quad 3.012 e-12 \quad 3.148 e-12 \quad 3.031 e-12 \quad 3.068 e-12$ 7.510e-01 3.219e-12 3.302e-12 3.266e-12 $3.411 e-12 \quad 3.279 e-12 \quad 3.317 e-12$

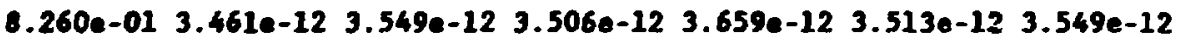
9.090e-01 3.734e-12 3.827e-12 $3.778 e-12 \quad 3.942 e-12 \quad 3.782 e-12 \quad 3.818 e-12$ $1.000 e+00 \quad 4.005 e-12 \quad 4.104 e-12 \quad 4.050 e-12 \quad 4.224 e-12 \quad 4.049 e-12 \quad 4.086 e-12$ $1.120 e+00 \quad 4.375 e-12 \quad 4.482 e-12 \quad 4.421 e-12 \quad 4.609 e-12 \quad 4.417 e-12 \quad 4.455 e-12$ $1.270 e+00 \quad 4.872 e-12 \quad 4.991 e-12 \quad 4.921 e-12 \quad 5.130 e-12 \quad 4.915 e-12 \quad 4.955 e-12$ $1.420 e+005.337 e-12 \quad 5.466 e-12 \quad 5.388 e-12 \quad 5.616 e-12 \quad 5.380 e-12 \quad 5.422 e-12$ $1.600 e+00 \quad 5.770 e-12 \quad 5.909 e-12 \quad 5.823 e-12 \quad 6.069 e-12 \quad 5.814 e-12 \quad 5.857 e-12$ $\begin{array}{lllllll}1.800 e+00 & 6.319 e-12 & 6.473 e-12 & 6.380 e-12 & 6.650 e-12 & 6.372 e-12 & 6.420 e-12\end{array}$ $2.030 e+00 \quad 6.869 e-12 \quad 7.039 e-12 \quad 6.939 e-12 \quad 7.236 e-12 \quad 6.936 e-12 \quad 6.990 e-12$ $2.280 e+007.582 e-12 \quad 7.774 e-12 \quad 7.669 e-12 \quad 8.001 e-12 \quad 7.673 e-12 \quad 7.736 e-12$ $2.570 e+008.438 e-128.659 e-12 \quad 8.547 e-12 \quad 8.924 e-12 \quad 8.565 e-12 \quad 8.642 e-12$ 2.890e+00 9.305e-12 9.558e-12 $9.444 e-12 \quad 9.870 e-12 \quad 9.482 e-12 \quad 9.576 e-12$ 3.250e+00 $1.023 e-11 \quad 1.052 e-11 \quad 1.041 e-11 \quad 1.089 e-11 \quad 1.047 e-11 \quad 1.059 e-11$ $3.650 e+001.140 e-11 \quad 1.174 e-11 \quad 1.163 e-11 \quad 1.219 e-11 \quad 1.174 e-11 \quad 1.188 e-11$ $4.110 e+00 \quad 1.268 e-11 \quad 1.308 e-11 \quad 1.297 e-11 \quad 1.362 e-11 \quad 1.313 e-11 \quad 1.331 e-11$ $4.620 e+001.426 e-11 \quad 1.473 e-11 \quad 1.464 e-11 \quad 1.538 e-11 \quad 1.486 e-11 \quad 1.508 e-11$ $5.200 e+001.607 e-11 \quad 1.663 e-11 \quad 1.655 e-11 \quad 1.742 e-11 \quad 1.686 e-11 \quad 1.714 e-11$ $5.850 e+001.813 e-11 \quad 1.879 e-11 \quad 1.873 e-11 \quad 1.975 e-11 \quad 1.914 e-11 \quad 1.949 e-11$ $6.580 e+002.054 e-112.132 e-11 \quad 2.129 e-11 \quad 2.248 e-11 \quad 2.181 e-112.224 e-11$ $\begin{array}{lllllll}7.410 e+00 & 2.353 e-11 & 2.447 e-11 & 2.447 e-11 & 2.588 e-11 & 2.515 e-11 & 2.568 e-11\end{array}$ 


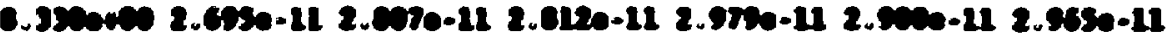

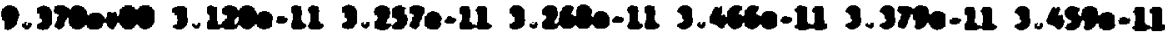

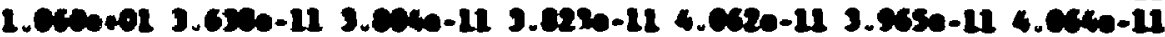

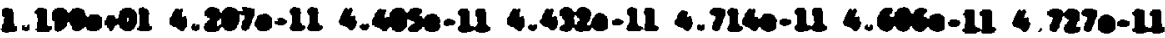

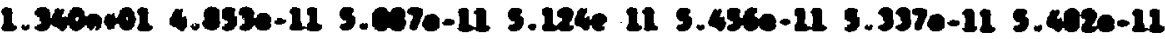

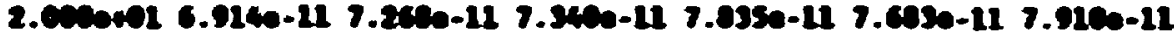

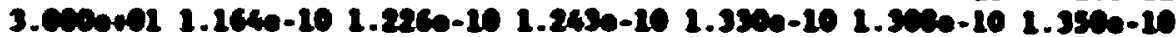

4.c00enel $1.7760-101.0750-101.5030-102.0420-102.0120-102.0750-10$

$5.60004012 .4340-102.5710-102.6120-102.6060-102.7650-102.054 e-10$

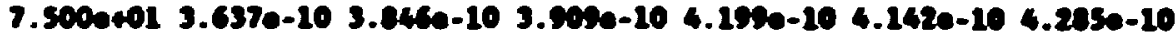

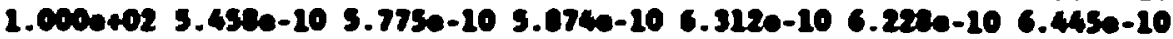




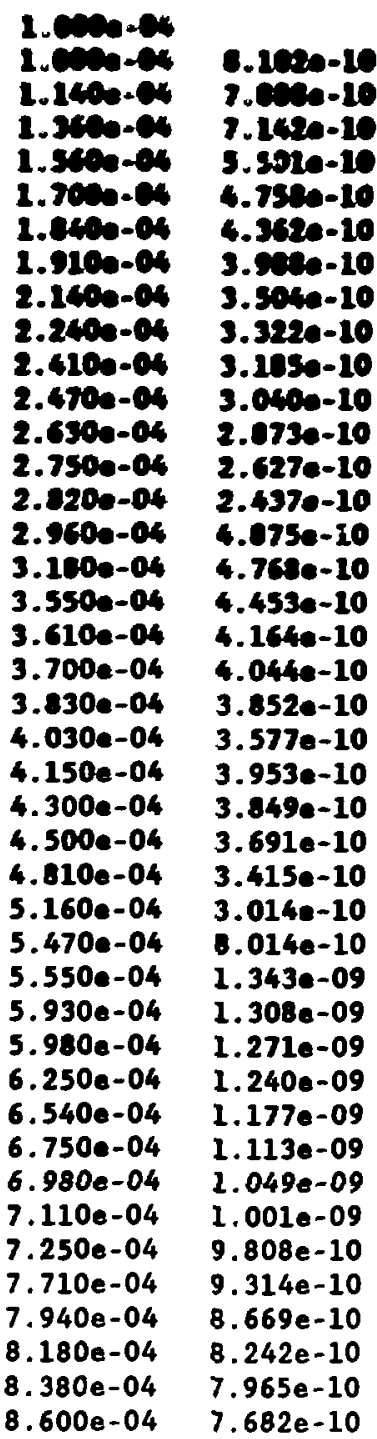

\author{
$0.23 \times-10$ \\ $0.2 \mathrm{men}-10$ \\ 5.7340-10 \\ s.0ste-10 \\ 5. $4020-10$ \\ S.170e-10 \\ 4.916e-10 \\ 4.640e-10 \\ 4.440e-10 \\ 4.2620-10 \\ 4.0500-10 \\ 3.920-10 \\ 3.6640-10 \\ 3. $4630-10$ \\ 8.185e-10 \\ a.205a-10 \\ $8.257 e-10$ \\ 1.014e-09 \\ 1.000e-09 \\ 9.696e-10 \\ 9.120e-10 \\ 9.129e-10 \\ 8.859e-10 \\ $8.728 e-10$ \\ 8. 304e-10 \\ 7.448e-10 \\ 9.922e-10 \\ 1.280e-09 \\ 1.229e-09 \\ $1.176 e-09$ \\ 1.142e-09 \\ 1.086e-09 \\ 1.027e-09 \\ $9.689 e-10$ \\ $9.250 \mathrm{e}-10$ \\ 9.062e-10 \\ 8. $596 e-10$ \\ $7.988 e-10$ \\ $7.588 \mathrm{e}-10$ \\ $7.333 e-10$ \\ $7.074 e-10$
}

Page -26-

\author{
C.173e-10 \\ $7.9570-20$ \\ $7.2120-20$ \\ 5.550-10 \\ 4.0x6-10 \\ 4.5710-10 \\ 4. $1420-10$ \\ 3.594-10 \\ 3.5120-10 \\ $3.3770-10$ \\ $3.230-10$ \\ 3.058- -10 \\ 2. $050-10$ \\ 2. $6050-10$ \\ $4.573 e-10$ \\ 4. 45se-10 \\ 4.186e-10 \\ 3. $913 e-10$ \\ 3. $799 e-10$ \\ 3.621e-10 \\ 3. 370 e -10 \\ $3.762 e-10$ \\ 3. $663 e-10$ \\ 3. $514 e-10$ \\ 3.253e-10 \\ 2.873e-10 \\ 7. $983 e-10$ \\ 1. 350e-09 \\ 1. 317e-09 \\ 1.281e-09 \\ $1.249 e-09$ \\ 1.186e-09 \\ 1.121e-09 \\ $1.057 e-09$ \\ $1.008 \mathrm{e}-09$ \\ 9.884e-10 \\ 9. $388 e-10$ \\ 8. $740 e-10$ \\ 8. $311 e-10$ \\ 8.031e-10 \\ $7.747 e-10$
}

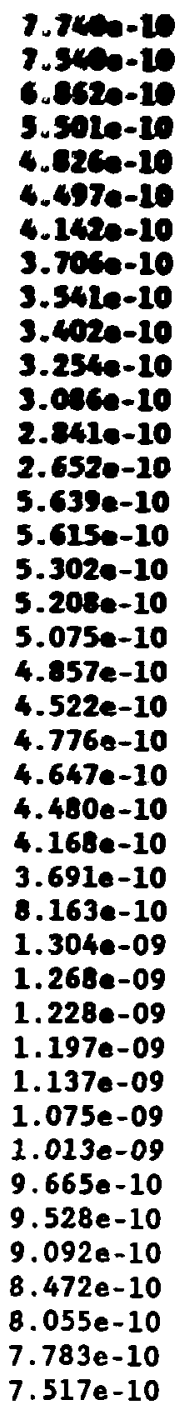


Va a a o u u u u U

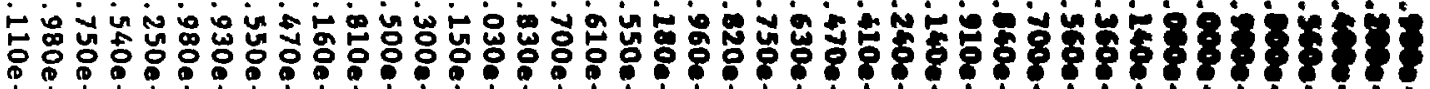

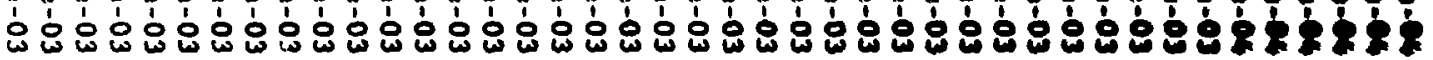

מ

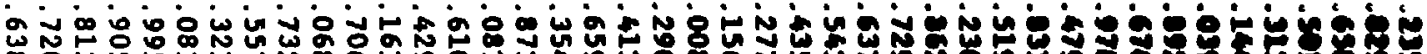
D.

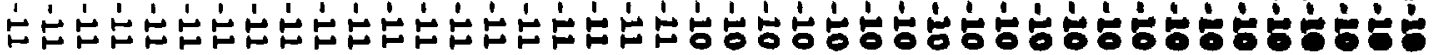

a

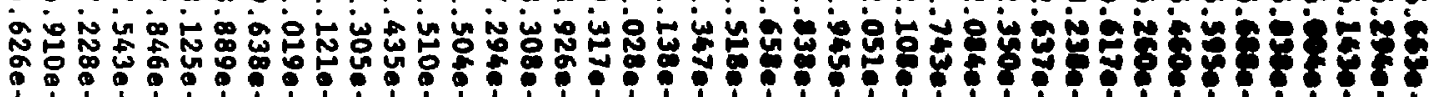

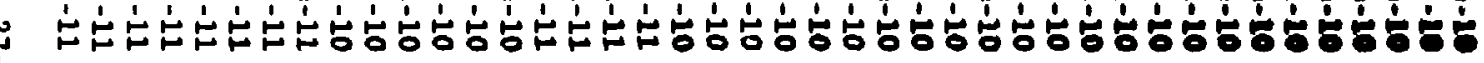

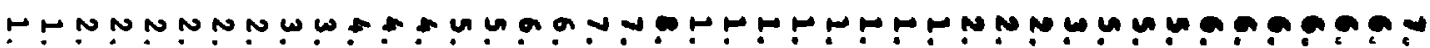

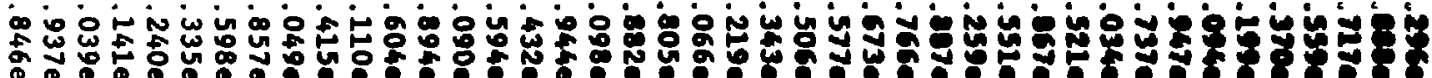

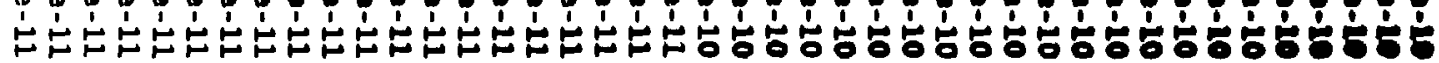

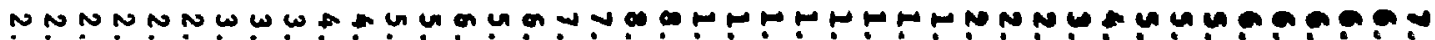

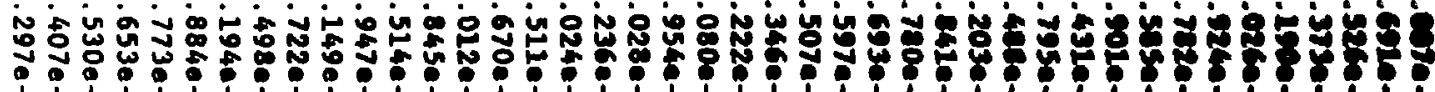

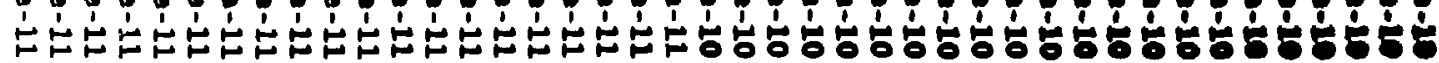




\section{$\cos$}

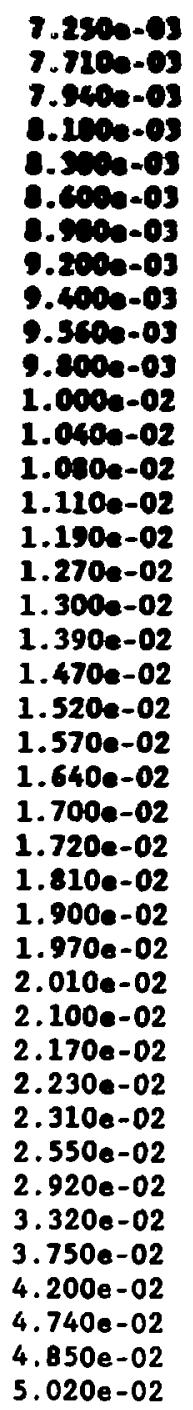

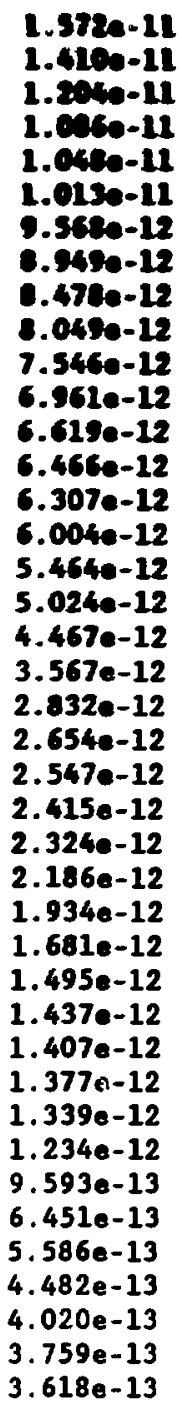

$6.395 e-11$
$5.631 e-11$
$5.110 e-11$
$4.691 e-11$
$4.551 e-11$
$4.417 e-11$
$4.204 e-11$
$3.967 e-11$
$3.706 e-11$
$3.621 e-11$
$3.427 e-11$
$3.201 e-11$
$3.067 e-11$
$3.005 e-11$
$2.939 e-11$
$2.811 e-11$
$2.582 e-11$
$2.395 e-11$
$2.156 e-11$
$1.769 e-11$
$1.451 e-11$
$1.371 e-11$
$1.320 e-11$
$1.256 e-11$
$1.212 e-11$
$1.146 e-11$
$1.024 e-11$
$9.005 e-12$
$8.100 e-12$
$7.806 e-12$
$7.635 e-12$
$7.470 e-12$
$7.264 e-12$
$6.682 e-12$
$5.180 e-12$
$3.433 e-12$
$2.856 e-12$
$2.126 \theta-12$
$1.746 e-12$
$1.517 e-12$
$1.401 e-12$

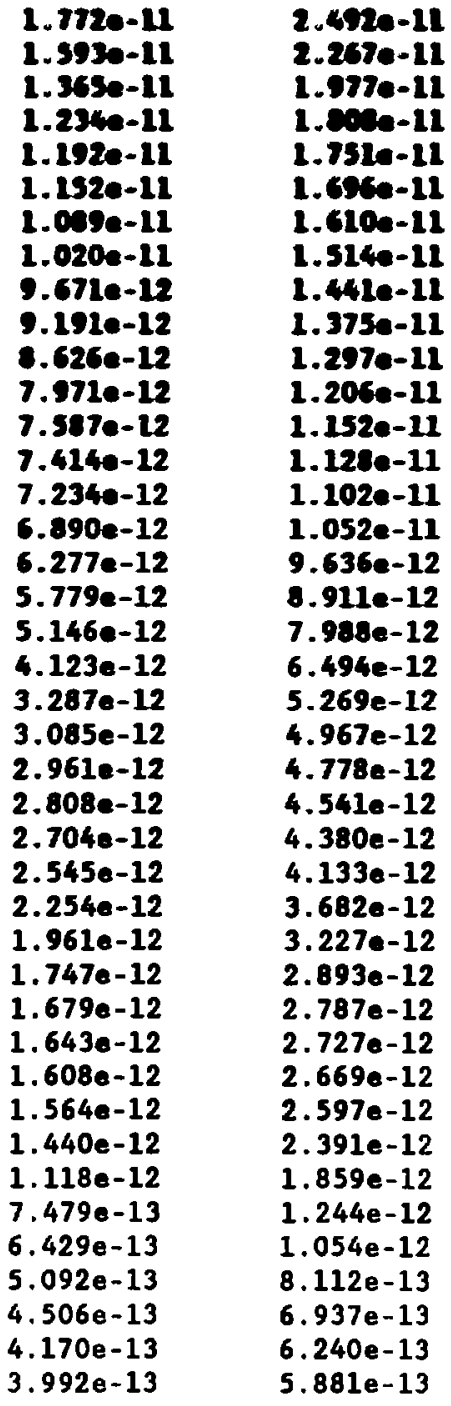




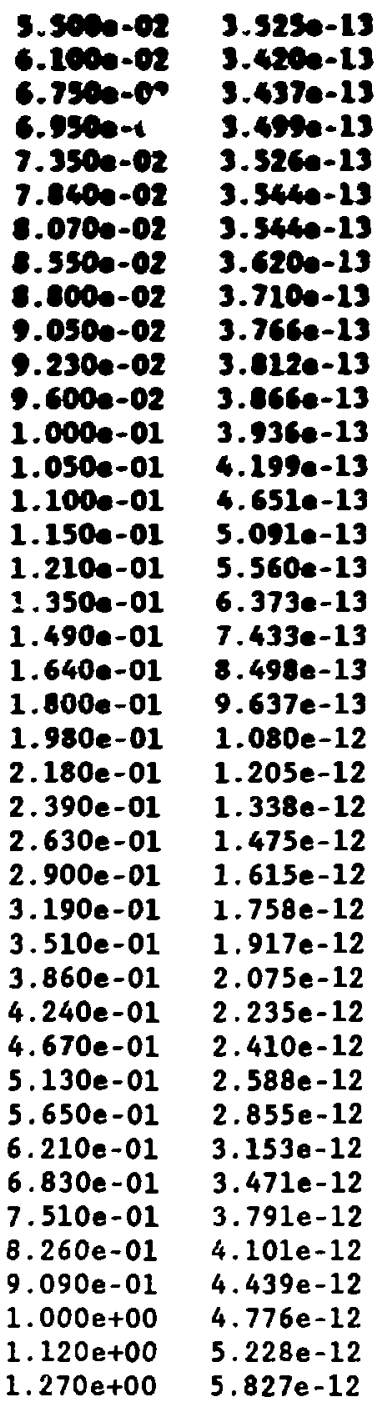

\author{
$1.2740-12$ \\ 1.012e-12 \\ $9.725 \mathrm{se}-13$ \\ $9.242 e-13$ \\ C.002e-13 \\ $0.0190-13$ \\ $7.3010-13$ \\ $7.1010-13$ \\ $6.9720-13$ \\ $6.070-13$ \\ $6.763 e-13$ \\ 6. $605 e-13$ \\ $6.349 a-13$ \\ $6.395 e-13$ \\ $6.763 e-13$ \\ 7. 103e-13 \\ $7.443 e-13$ \\ $7.967 \bullet-13$ \\ 8.521e-13 \\ 9.190e-13 \\ 1.017e-12 \\ $1.112 e-12$ \\ 1. 219e-12 \\ 1. $344 e-12$ \\ 1. $469 e-12$ \\ 1. 595e-12 \\ 1. $723 e-12$ \\ 1.873e-12 \\ 2.023e-12 \\ 2.172e-12 \\ 2.340e-12 \\ 2. 509e-12 \\ 2.765e-12 \\ 3. 052e-12 \\ 3. 357e-12 \\ 3. $665 e-12$ \\ 3. $963 e-12$ \\ 4. $289 e-12$ \\ 4. $613 e-12$ \\ 5. $049 e-12$ \\ 5. 628e-12
}

$5.536 e-13$
$5.052 e-13$
$4.018 e-13$
$4.763 e-13$
$4.650-13$
$4.536 e-13$
$4.391 e-13$
$4.39 e-13$
$4.442 e-13$
$4.463 e-13$
$4.476 e-13$
$4.455 e-13$
$4.455 e-13$
$4.703 e-13$
$5.139 e-13$
$5.560 e-13$
$6.005 e-13$
$6.760 e-13$
$7.717 e-13$
$8.704 e-13$
$9.816 e-13$
$1.094 e-12$
$1.216 e-12$
$1.348 e-12$
$1.483 e-12$
$1.621 e-12$
$1.762 e-12$
$1.919 e-12$
$2.077 e-12$
$2.235 e-12$
$2.410 e-12$
$2.588 e-12$
$2.854 e-12$
$3.152 e-12$
$3.469 e-12$
$3.788 e-12$
$4.097 e-12$
$4.435 e-12$
$4.771 e-12$
$5.223 e-12$
$5.822 e-12$

$3.85 e-13$
$3.659-13$
$3.653 e-13$
$3.706 e-13$
$3.716 e-13$
$3.707 e-13$
$3.692 e-13$
$3.746 e-13$
$3.630 e-13$
$3.061 e-13$
$3.922 e-13$
$3.969 e-13$
$4.028 e-13$
$4.284 e-13$
$4.735 e-13$
$5.172 e-13$
$5.638 e-13$
$6.443 e-13$
$7.469 e-13$
$8.544 e-13$
$9.681 e-13$
$1.084 e-12$
$1.208 e-12$
$1.342 e-12$
$1.478 e-12$
$1.619 e-12$
$1.762 e-12$
$1.920 e-12$
$2.079 e-12$
$2.238 e-12$
$2.414 e-12$
$2.593 e-12$
$2.860 e-12$
$3.159 e-12$
$3.477 e-12$
$3.797 e-12$
$4.107 e-12$
$4.446 e-12$
$4.784 e-12$
$5.236 e-12$
$5.837 e-12$




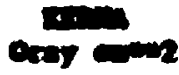

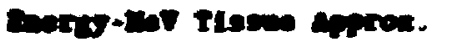

Eave (Rem)

Inecle (rou)

ste. aㅔ

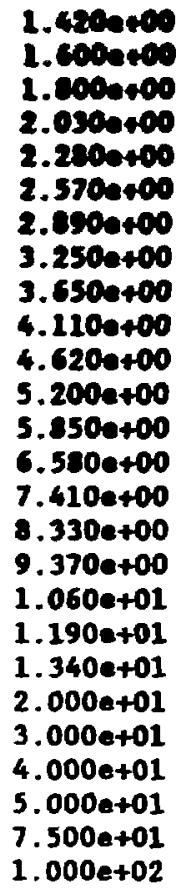
6.3950-12
6.097e-12
7.51\%-12
0.110e-12
c.854a-12
$0.7000-12$
1.046-11
1.122e-11
1.216a-11
1.305e-11
1.413e-11
$1.527 e-11$
1.652e-11
1.790e-11
$1.959 e-11$
2.227e-11
2.334e-11
2.569e-11
2. $842 e-11$
3.132e-11
3. $968 e-11$
5.859e-11
8.203e-11
1.068e-10
1.523e-10
2.209e-10

$6.167 e-12$
$6.653 e-12$
$7.267 e-12$
$7.967 e-12$
$8.579 e-12$
$9.410 e-12$
$1.020 e-11$
$1.097 e-11$
$1.193 e-12$
$1.286 e-11$
$1.401 e-11$
$1.524 e-11$
$1.660 e-11$
$1.821 e-11$
$1.998 e-11$
$2.189 e-11$
$2.425 e-11$
$2.699 e-11$
$3.013 e-11$
$3.352 e-11$
$4.360 e-11$
$6.650 e-11$
$9.529 e-11$
$1.259 e-10$
$1.819 e-10$
$2.666 e-10$

6. 396- -12

6.379e-12

6. 5020-12

6.091e-12

7.5310-12

7.512e-12

8.1240-12

- 106-12

$8.009 e-12$

- . e4se-12

$9.717 \bullet-12$

9.697e-12

1.050e-11

1.048e-11

1. 225e-11

1. 123e-11

$1.219 e-11$

1. 218e-11

1. $300-11$

1. $307 e-11$

1. $4170-11$

1. $417 e-12$

1.532e-11

1.533e-11

1. 658e-11

1.660e-11

1. 797e-11

1.801e-12

1.968e-11

1. 973e-11

2.137e-11

2.145e-11

2.346e-11

2.357e-11

2. 583e-11

2.599e-11

2.859e-11

2.879e-11

3.153e-11

$3.178 e-11$

4.000e-11

4.043e-11

5.917e-11

6.001e-11

8.295e-11

8. 434e-11

1.081e-10

1. 101e-10

$1.542 e-10$

$1.573 e-10$

2.239e-10

2. 286e-10

Page - 30 - 
trefte Hig. Hase. In.

1.000e-04

1.000-04

$1.1400-04$

1.36e-04

1.350-04

$1.7000-04$

$1.0400-04$

1.910e-04

2.140e-04

2.2400-04

$2.4100-04$

$2.4700-04$

2.630e-04

$2.7500-04$

2.820e-04

2.960e-04

3.180e-04

3.550e-04

3.610e-04

3. $700 e-04$

3.830e-04

4.030e-04

4.150e-04

4. 300e-04

4.500e-04

4.810e-04

5.160e-04

5.470e-04

5.550e-04

5.930e-04

5. 980 e- 04

6. 250 e- 04

6. 540e-04

6. 750 e-04

6. $980 e-04$

7.110e-04

7. $250 e-04$

7.710e-04

7. $940 e-04$

8. $180 e-04$

8. 380 e- 04

8. $600 e-04$
$4.5100-10$

4.3440-10

3.95se-10

3.04he-10

2.636- -10

2.419e-10

2.215e-10

1. 94e- 10

1. $842 e-10$

1. $758 e-10$

1.671e-10

1.572e-10

1. $429 e-10$

1. $318 e-10$

1.506e-09

1. 583e-09

$1.497 e-09$

$1.439 e-09$

$1.403 e-09$

1. 344e-09

1.249 e-09

1. $242 e-09$

1. 208e-09

$1.161 e-09$

$1.076 \mathrm{e}-09$

9.496e-10

$9.134 \mathrm{e}-10$

9.121e-10

$8.537 e-10$

$7.938 \mathrm{e}-10$

$7.654 \mathrm{e}-10$

7. 246e-10

6.829e-10

$6.539 e-10$

$6.332 \mathrm{e}-10$

$6.190 \mathrm{e}-10$

$5.840 \mathrm{e}-10$

5. 386e-10

5. $090 \mathrm{e}-10$

$4.909 \mathrm{e}-10$

$4.726 \mathrm{e}-10$ 5.113e-10

$4.9690-10$

$4.4400-10$

3. $426-10$

2.955-10

2.705e-10

2.473e-10

2.169e-10

2.050e-10

1.958e-10

1.862e-10

1.753e-10

1.594e-10

$1.471 \mathrm{e}-10$

$1.272 e-09$

$1.330 e-09$

1.251e-09

1.177e-09

$1.146 e-09$

$1.096 e-09$

$1.029 e-09$

$1.181 \mathrm{e}-09$

1.150e-09

1. 104e-09

1.023e-09

9.058e-10

9. $392 \theta-10$

1. $009 e-09$

9.518e-10

8. $926 e-10$

8. $626 e-10$

8.170e-10

$7.703 e-10$

7. 241e-10

6.891e-10

$6.739 e-10$

6. $361 \mathrm{e}-10$

5. $872 e-10$

5. 553e-10

5. $358 \mathrm{e}-10$

5. $160 \mathrm{e}-10$ 5.814e-10

5.657e-10

5.116-10

3.932e-10

$3.397 e-10$

3.113e-10

2. 847e-10

2. $4990-10$

2.365e-10

2.261e-10

2.153e-10

2.028 - 10

$1.847 e-10$

1. 706e-10

1.227e-09

1.278e-09

1. 202e-09

1.130e-09

1. 100e-09

$1.052 e-09$

9.720e-10

9.194e-10

8.944e-10

8. 566e-10

7.907e-10

6.954e-10

8.737e-10

1.090e-09

1. $041 e-09$

9.892e-10

9.591e-10

9.092e-10

8.579e-10

8. 072e-10

$7.689 e-10$

$7.524 \theta-10$

7. $117 \mathrm{e}-10$

$6.587 e-10$

$6.240 \mathrm{e}-10$

$6.023 e-10$

$5.802 e-10$ 8.250e-10

0.032e-10

7. 273e-10

5. $603 e-10$

$4.847 e-10$

$4.4430-10$

4.063e-10

3.570e-10

3.385e-10

3.245e-10

3.098e-10

2.927e-10

$2.678 e-10$

$2.485 e-10$

4. $396 e-10$

4.249e-10

$3.963 e-10$

3.701e-10

3. $593 e-10$

3. $420 e-10$

$3.177 e-10$

3. $584 e-10$

3. $489 e-10$

3. $347 e-10$

3. 098e-10

$2.734 e-10$

$7.952 e-10$

$1.358 e-09$

$1.325 e-09$

$1.289 e-09$

$1.258 e-09$

$1.194 \mathrm{e}-09$

$1.129 e-09$

1.064e-09

$1.016 e-09$

$9.954 e-10$

$9.455 e-10$

8. $803 e-10$

8. $371 e-10$

8.090e-10

7. $803 e-10$

Page -31- 
Liq. Nave. Ay.

\begin{tabular}{ll}
$8.960 e-04$ & $4.437 e-10$ \\
$7.200 e-04$ & $4.174 e-10$ \\
$9.400 e-04$ & $4.659 e-10$ \\
$9.560 e-04$ & $3.954 e-10$ \\
$9.800 e-04$ & $3.02 e e-10$ \\
$9.900 e-04$ & $3.715 e-10$ \\
$1.000 e-03$ & $3.645 e-10$ \\
$1.090 e-03$ & $3.549 e-10$ \\
$1.140 e-03$ & $3.412 e-10$ \\
$1.360 e-03$ & $2.975 e-10$ \\
$1.560 e-03$ & $2.042 e-10$ \\
$1.700 e-03$ & $1.643 e-10$ \\
$1.840 e-03$ & $1.455 e-10$ \\
$1.910 e-03$ & $1.281 e-10$ \\
$2.140 e-03$ & $1.060 e-10$ \\
$2.240 e-03$ & $9.815 e-11$ \\
$2.410 e-03$ & $9.277 e-11$ \\
$2.470 e-03$ & $8.726 e-11$ \\
$2.630 e-03$ & $8.096 e-11$ \\
$2.750 e-03$ & $7.178 e-11$ \\
$2.820 e-03$ & $6.475 e-11$ \\
$2.960 e-03$ & $5.614 e-11$ \\
$3.180 e-03$ & $4.577 e-11$ \\
$3.550 e-03$ & $4.078 e-11$ \\
$3.610 e-03$ & $3.651 e-11$ \\
$3.700 e-03$ & $3.479 e-11$ \\
$3.830 e-03$ & $3.208 e-11$ \\
$4.030 e-03$ & $2.764 e-11$ \\
$4.150 e-03$ & $3.544 e-11$ \\
$4.300 e-03$ & $3.481 e-11$ \\
$4.500 e-03$ & $3.283 e-11$ \\
$4.810 e-03$ & $2.945 e-11$ \\
$5.160 e-03$ & $2.469 e-11$ \\
$5.470 e-03$ & $2.215 e-11$ \\
$5.550 e-03$ & $2.081 e-11$ \\
$5.930 e-03$ & $1.900 e-11$ \\
$5.980 e-03$ & $1.715 e-11$ \\
$6.250 e-03$ & $1.649 e-11$ \\
$6.540 e-03$ & $1.579 e-11$ \\
$6.750 e-03$ & $1.507 e-11$ \\
$6.980 e-03$ & $1.434 e-11$ \\
$7.110 e-03$ & $2.369 e-11$ \\
\hline &
\end{tabular}

$4.067 e-10$
$4.562 e-10$
$4.439 e-10$
$4.326 e-10$
$4.191 e-10$
$4.070 e-10$
$3.995 e-10$
$3.091 e-10$
$3.742 e-10$
$3.265 e-10$
$2.245 e-10$
$1.808 e-10$
$1.602 e-10$
$1.411 e-10$
$1.169 e-10$
$1.082 e-10$
$1.023 e-10$
$9.619 e-11$
$8.925 e-11$
$7.915 e-11$
$7.141 e-11$
$6.193 e-11$
$5.050 e-11$
$4.500 e-11$
$4.029 e-11$
$3.839 e-11$
$3.539 e-11$
$3.050 e-11$
$2.754 e-11$
$2.643 e-11$
$2.479 e-11$
$2.200 e-11$
$1.810 e-11$
$1.60 e, e-11$
$1.500 e-11$
$1.358 e-11$
$1.213 e-11$
$1.162 e-11$
$1.108 e-11$
$1.053 e-11$
$9.985 e-12$
$9.497 e-12$
3.0

$3.4540-10$

$5.137 e-10$

5.001e-10

4.075e-10

4. $725 \%-10$

$4.5910-10$

4. 501e-10

$4.393 e-10$

$4.227 e-10$

3.694e-10

2.555e-10

2.065e-10

$1.832 e-10$

$1.617 e-10$

1. $343 e-10$

1.245e-10

1.177e-10

$1.108 e-10$

1.029e-10

9.137e-11

8. 253e-11

7.172e-11

5.866e-11

5.233e-11

4.692e-11

$4.473 e-11$

$4.128 e-11$

3. $563 e-11$

3. $222 e-11$

$3.093 a-11$

2. $903 e-11$

$2.580 e-11$

$2.127 e-11$

1.890e-11

$1.767 e-11$

$1.601 e-11$

$1.432 e-11$

1. $372 e-11$

1. $309 \mathrm{e}-11$

1. $245 \mathrm{e}-11$

$1.181 e-11$

1. $124 \mathrm{e}-11$
7. $349 e-10$

$0.030-10$

$6.755 e-10$

6. $605 e-10$

$6.415 e-10$

6. $.93 e-10$

6.131e-10

5. $908 \mathrm{e}-10$

5.769e-10

5.061e-10

3.538e-10

2.881e-10

$2.563 e-10$

2.269e-10

$1.895 e-10$

1. 760 e- 10

1. 667e-10

1.571e-10

1.461e-10

1. 301e-10

$1.178 \mathrm{e}-10$

$1.027 e-10$

$8.447 e-11$

$7.554 e-11$

$6.787 \mathrm{e}-11$

$6.476 e-11$

$5.987 e-11$

5. 186e-1:

4. 701e-11

4. $516 \mathrm{e}-11$

$4.243 e-11$

$3.779 e-11$

3. $129 e-11$

2.786e-11

$2.608 e-11$

2. $367 e-11$

$2.123 e-11$

2. $035 e-11$

$1.944 \mathrm{e}-11$

$1.850 \mathrm{e}-11$

1. $756 \mathrm{e}-11$

$1.672 e-11$

Page - 32 - 


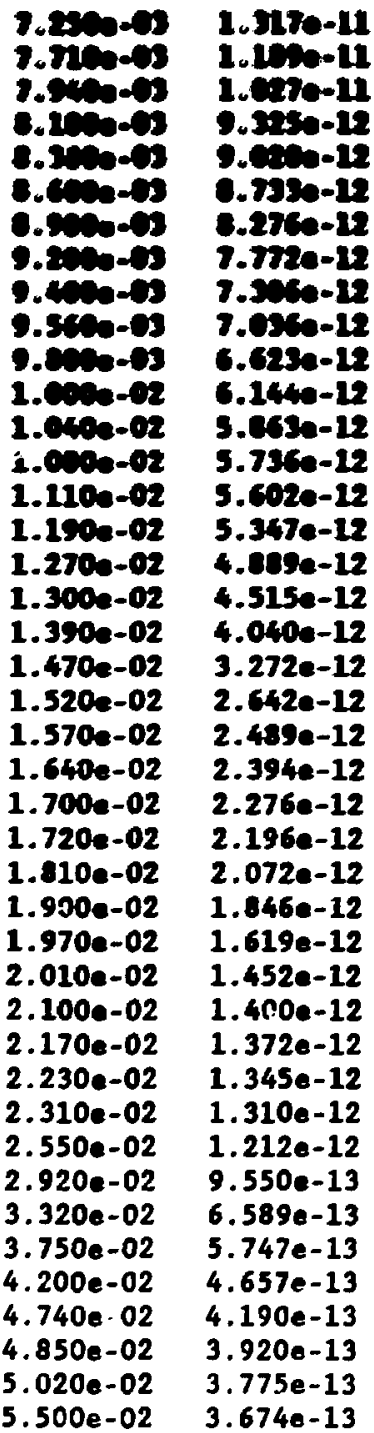

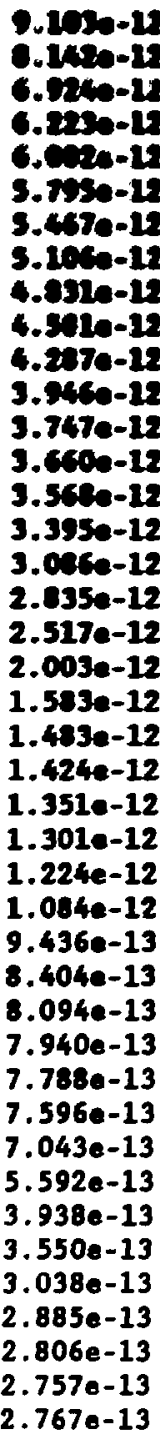

Lucho-21

- c4re-12

c.217e-12

$7.3 \times 4-12$

$7.13 \times-12$

6.6xe-12

$6.5080-12$

6.077e-12

$3.7520-22$

5.4570-12

$5.1110-12$

4.705e-12

$4.473 e-12$

4.35se-12

4.261e-12

4.054e-12

3. $677 e-12$

3.39se-12

3.010e-12

2.358e-12

1.895e-12

1.779e-12

1.708- -12

1. 619e-12

1.559e-12

.. $467 e-12$

1.299-12

1.130--12

1.005e-12

9.674e-13

$9.481 \mathrm{e}-13$

9.291e-13

9.053e-13

8. 371e-13

6.591e-13

4.557e-13

4.046e-13

3. $381 e-13$

$3.146 e-13$

$3.018 e-13$

2. $945 e-13$

2. $928 e-13$
3.

1. $4310-11$

1.2040-11

$12 k-11$

1.0015-21

1.0.0-11

$0.767 e-12$

0.235-12

e.65se-12

0.21e-12

$7.7040-12$

7.107-22

$6.755 e-12$

6. $0030-12$

6. 4400-22

6.130e-12

5.57se-12

5.131e-12

4.562e-12

3.643e-12

2.893e-12

2.711e-12

2.602e-12

2.467e-12

2.374e-12

2.233e-12

1.976e-12

1.717e-12

$1.527 a-12$

$1.468 e-12$

$1.436 a-12$

1. 406a-12

1. 368 - 12

1. 259e-12

9.790e-13

$6.575 e-13$

5. $687 \mathrm{e}-13$

4.555e-13

4.078e-13

3. 808e-13

$3.663 e-13$

$3.565 e-13$

Page -33- 


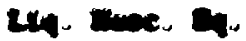

0.100.

$0.7+1.0$

s.sench

7.38 .

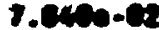

0.Oinatir

0.5rive.er

B. Drivere

9.08c-

9.2310.

9.coser.

2.0.6-01

1.050-01

1.102-01

1.150-01

1.2100-01

1.3500-01

1. $4500-01$

1.640e-01

1.800e-01

1.980e-01

2.180e-01

2.390e-01

2.630e-01

2.900--01

3.190e-01

3.510e-01

3.860e-01

4.240e-01

4.670e-01

5.130e-01

5.650e-01

6.210e-01

6.830e-01

7. 510e-01

8. 260e-01

9.090e-01

1. 000et00

1.120 et 00

1. $270 e+00$
$3.5580-25$

$3.5570-23$

$3.020-13$

$3.0325-13$

3.cis- 13

3.02. 13

$3.301 \mathrm{e}-13$

3. $207-13$

3. $1 \times 1-13$

$3.092-19$

$3.933-23$

$3.5970-13$

4.250 - 13

$4.707 e-13$

5.245e-13

$5.6130-13$

6. 420 - 13

7. 45 e -13

8.526e-13

$0.662 \mathrm{e}-13$

1.092-12

1.206e-12

1.340--12

$1.476 \mathrm{e}-12$

1.616e-12

1.758e-12

1.917e-12

2.075e-12

2. 234e-12

2. 410--12

2. 588- -12

$2.854 \mathrm{e}-12$

$3.153 e-12$

3. 470e-12

3.790e-12

4. 100e-12

$4.438 e-12$

4. $775 \mathrm{e}-12$

$5.227 e-12$

5. $826 e-12$
2. $1020-13$

2.5est 13

3.035-13

3.10a-13

3.11 $5-13$

$3.243-10$

3. 319-13

3. 사- 23

3. 3250-13

$3.5720-13$

3. $(4)-13$

$3.7370-13$

4.0170-13

4.473e-13

4.919e-13

5.396 e-13

$6.226-13$

$7.319-13$

8.409e-13

$9.553 \mathrm{e}-13$

1.072e-12

1. 198- -12

1. 332e-12

$1.468-12$

1. 609e-12

1.752e-12

1.910e-12

2.069e-12

2. 228- -12

2.403e-12

2. 581e-12

2. 847 e -12

$3.145 e-12$

$3.462 \mathrm{e}-12$

3. 781 e-12

4.090e-12

4. $428 \mathrm{e}-12$

4.764e-12

$5.215 e-12$

$5.813 e-12$
$2.9 \mathrm{na}-23$

3.012. 43

$3.2110 \cdot 23$

3. $255-13$

$3.24-13$

$3.2710-13$

3.3510-13

3.45n-23

$3.525-13$

3.574- 13

3. $4440-13$

3.736- 13

4.001-13

4.44e-13

$4.995-13$

5.352 - 13

6.164e-13

7. $230 \mathrm{n}-13$

‥295e-13

$9.420 \mathrm{e}-13$

1.057e-12

1. 180e-12

1. 312 e-12

$1.446 e-12$

$1.584 \mathrm{e}-12$

1.725 - 12

1.081e-12

2.037e-12

2.193--12

2.366e-12

2. 541e-12

2.803e-12

3. 096e-12

3. $408 \mathrm{e}-12$

$3.722 \mathrm{e}-12$

$4.026 e-12$

$4.359 \mathrm{e}-12$

$4.689 e-12$

$5.133 e-12$

5. $722 \mathrm{e}-12$
$3.4530-12$

$3.455-13$

$3.525-13$

$3.5510-23$

3.5xte-13

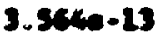

$3.6320-23$

$3.7270-13$

$3.703-13$

$3.029-13$

$3.839-13$

$3.0510-13$

4.214-13

4. $656-23$

5.106e-13

5.576e-13

6.359e-13

7. $449 e-13$

$8.515 e-13$

$9.656 e-13$

1.002e-12

1. 207e-12

1. 341e-12

1.477 e-12

$1.618 e-12$

1.761 e-12

1.920 e- 12

2.079e-12

2.238 e- 12

2. 414e-12

2.592e-12

2.860e-12

$3.159 e-12$

$3.477 e-12$

$3.797 e-12$

4. 108 e -12

$4.446 e-12$

4.784e-12

$5.237 e-12$

$5.837 e-12$

Page -34- 


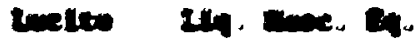

1. Sinowern

1. conowin

1. Comenters

2.0Bern

2.820040)

2.57 wores

2.03nows

$3.250 \mathrm{sich}$

$3.6800+00$

0.1100400

4.6200400

$5.2000+00$

$5.0500+00$

$0.5000+00$

$7.4100+00$

$8.3300+00$

$9.3700+60$

1.060et01

$1.190 e+01$

1. 340e+01

2.000e+01

3.000e+01

$4.000 e+01$

5.000e+01

$7.500 e+01$

1.000e+02
0.3330.12

c.coso-12

7.525e-12

0.1010-12

c.0sso-12

9.675-12

1.066-12

1.1170-12

1.205e-12

1.293e-11

1.398e-11

1.506e-11

1.624e-11

1.755e-11

1. $913 e-11$

2.05e-11

2.259-11

2. $4746-11$

2.725e-11

2.990e-11

3.739e-11

5.429e-11

7.504e-11

9.691e-11

1. 370 e-10

$1.974 e-10$
6.390-12

6.075-12

7.47)-12

-.012-12

$0.0140-12$

$0.6580-12$

2.0420-12

2.1160-21

1.205e-11

1.250-11

1.396e-11

1.502e-11

1.621e-11

1.751e-11

1. 00 e-11

2.063e-11

2.254e-11

2.45e-11

2.719e-11

2.983e-11

3.731e-11

5.417e-11

7. 48ce-11

9.672e-11

1.367e-10

1.971e-10 6.24se-12

c.7me-12

7. 3000-12

7. $\operatorname{css}-12$

- cace-12

$0,5100-12$

1.0270-11

2.cese-12

1.155e-11

$1.276-11$

1.376e-11

1.457e-11

1.coce-11

1.737e-11

1. $097 e-11$

2.053e-11

2.247e-11

2.465e-11

2. 721 - 11

2.991e-11

3. $760 e-11$

5.497e-11

7. 638e-11

9.900e-11

1. 404e-10

2.030e-10 6.316e-12

6. Same-12

$7.5310-12$

0. 204-12

0.05se-12

9.716a-12

1. esco-11

1. $226-11$

1.2180-11

1.307e-11

1.4160-11

1.5300-11

1.656- 11

1.795e-11

1. Sc4e-11

2.132e-11

2. 340e-11

2.576e-11

2.050a-11

3.142e-11

3.982e-11

5. $863 e-11$

8.240e-11

1.073e-10

$1.530 e-10$

2.221e-10 


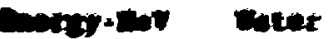

L.encesen

2. content

1.1400-0.

1. $300-4$

1. 5 revery

2.7000-0y

1.040e-c4

1.9100-94

2.1400-96

2.2400-04

$2.4100-04$

2. $4700-04$

2.630e-06

$2.7500-04$

2.020e-04

2.9c0-04

3.1000-04

3.5500-04

3.610e-04

3.700e-04

$3.830 \mathrm{e}-04$

4.030e-04

4.150e-04

4.300e-04

$4.500=-04$

4.8100-04

5.160e-04

5.470e-04

5.550e-04

5.930e-04

5.980e-04

6.250e-04

6.540e-04

6.750e-04

6.980e-04

7.110e-04

$7.250 e-04$

7.710e-04

$7.940 e-04$

8. $180 e-04$

8. $380 e-04$

$8,600 e-04$
- 4 45-10

c.726.10

$8.0030-10$

c. cane-10

5.257e-10

4.024e-10

$4.4160-10$

3. $0010-10$

3. cele-10

$3.3300-10$

3.372e-10

3.106-10

2. 9L1ㄴ-10

2.710e-10

2.452e-10

2.1360-10

$1.967 \mathrm{e}-10$

1.018e-10

$1.757 \mathrm{e}-10$

1.660e-10

$1.499 e-10$

1.400e-10

1. 360e-10

1. 300e-10

1. 196e-10

1.043e-10

7.455e-10

$1.429 e-09$

$1.402 e-09$

1. 373e-09

1.341e-09

$1.274 e-09$

1. 205e-09

$1.136 \mathrm{e}-09$

$1.085 e-09$

$1.064 e-09$

1.011 e-09

$9.429 e-10$

$8.974 \mathrm{e}-10$

$8.675 e-10$

8. $369 e-10$

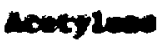

Nor nit

meter sot, whe

4.017e-10

3.003e-10

$3.325-10$

2. $\operatorname{cose-10}$

$2.320-10$

$2.1250-10$

$1.2420-10$

1.703e-10

1. Cose- 20

1.529e-10

1.449e-10

1. $3550-10$

$1.220-10$

1.126e-10

1.752e-09

1.845e-09

1.740e-0s

1.638e-0s

1.595e-09

1.527e-09

$1.412 e-09$

$1.337 e-09$

1.301e-09

$1.246 e-09$

1. 150e-09

1.012e-09

9. 314e-10

8.864e-10

8. $247 e-10$

7. $615 e-10$

7. 328e-10

$6.932 e-10$

$6.527 e-10$

6.127e-10

$5.822 e-10$

5. $686 \mathrm{e}-10$

5. $348 \mathrm{e}-10$

$4.912 \mathrm{e}-10$

$4.630 e-10$

4. $462 \mathrm{e}-10$

$4.293 e-10$
$7.026-10$

7. $420-10$

$6.7220-10$

$5.1336-10$

$4.426-10$

4.0se-10

3.705-10

$3.2560-10$

$3.0310-10$

2.967e-10

2.861e-10

3.021e-10

2.765e-10

2.552e-10

$2.327 e-10$

2.053e-10

$1.9240-10$

$1.7910-10$

$1.735 e-10$

1.646e-10

2. $454 e-10$

1.495e-09

1. 465e-09

1.417e-09

1.333e-09

1.207e-09

1. 2940-09

$1.425 e-09$

$1.352 e-09$

1.277e-09

$1.237 e-09$

$1.172 e-09$

1.107e-09

$1.042 e-09$

$9.925 e-10$

$9.717 e-10$

9.202e-10

8. $531 e-10$

$8.093 e-10$

7. $819 \mathrm{e}-10$

$7.541 e-10$
1. cure-10

7. $470-10$

$8.734-10$

$5.1470-10$

4.45e-10

4.035e-10

$3.725-10$

$3.265=-10$

$3.058-10$

2.975e-10

2.069e-10

3.023e-10

2.767e-10

2.555e-10

2.325e-10

2.054e-10

$1.925 e-10$

$1.791 e-10$

$1.735 e-10$

$1.646 e-10$

2.440e-10

$1.476 e-09$

$1.446 e-09$

1.399e-09

1.316e-09

1.191e-09

1.286e-09

$1.425 e-09$

1.353e-09

$1.279 e-09$

1.238e-09

1.174e-09

$1.108 e-09$

$1.043 e-09$

$9.939 e-10$

9. $731 \mathrm{e}-10$

9.215e-10

8. $545 e-10$

8. $106 \mathrm{e}-10$

$7.831 \mathrm{e}-10$

$7.553 e-10$

Page -36- 

Cond

ester

D.sen.e.

- $20 \mathrm{men}$

o.centas

9. seno-4y

9.cmo-y

9. sene-cy

2. ene-03

2.0.0.-03

2.1400-03

1.340e-03

1.540e-03

1.7000-03

1.400-03

1. 10 -03

2.1400-03

2.2400-03

2.410e-03

2.470e-03

2.630e-03

2.750e-03

2.620e-03

2.960e-03

3.180e-03

3.550e-03

3.610e-03

3.700e-03

3.830e-03

$4.030 e-03$

$4.150=-03$

4. $300 e-03$

$4.500=-03$

4.810e-03

5.160e-03

5.470e-03

5.550e-03

5.930e-03

5.980e-03

6.250e-03

6.540e-03

6.750 e-03

6.980e-03

7.110e-03
7. $010-10$

7. 4he -10

$7.2060-10$

7.c15-20

c.cose-10

c.71C- 10

0.5ine-20

6. 452e-10

$0.205 \mathrm{se} 10$

5. $446-10$

3.016-10

3.123e-10

2.7720-10

2. $456-10$

2.054e-10

1. s0se-10

$1.007 e-10$

1.704e-10

$1.525 e-10$

$1.4120-10$

$1.279 e-10$

1. 116e-10

9.194e-11

B.226e-11

7.393e-11

$7.056 e-11$

6.525e-11

5.657e-11

5.130e-11

4.929e-11

4. 633e-11

$4.128 e-11$

3. $420 \mathrm{e}-11$

3.048e-11

2.854e-11

2.591e-11

2.324e-11

2.229e-11

2.129e-11

2.026e-11

$1.923 e-11$

1. 832e-11


4.025e-10

3.700 .10

3.6710-10

3.572e-10

$3.4530-10$

$3.2470-10$

$3.2220-10$

$3.1330-10$

3.057e-10

2.657e-10

1.016- 10

1. $453 e-10$

$1.2240-10$

$1.127 e-10$

9.209e-11

- $.502 e-11$

6.102e-11

7.612e-11

$7.052 a-11$

$6.2380-11$

$5.615 e-11$

4.852e-11

3.934e-11

3. $497 e-11$

3.124e-11

2.974e-11

2.737e-11

2. 350e-11

2.117e-11

2.029e-11

1. 901e-11

$1.683 e-11$

1. 379e-11

1.220e-11

1.139e-11

1.028e-11

9.165e-12

8. $768 e-12$

8. 358 e-12

7. $936 \mathrm{e}-12$

$7.516 e-12$

$7.142 e-12$

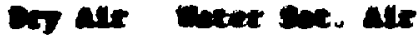

7.200.-10

6.720.10

6.535-20

6. 316-10

6.2010-10

6.03x-10

$3.9340-10$

$5.707 e-10$

5.5700-10

4.071e-10

3.375e-10

$2.732 \cdot-10$

2. $425-10$

2.141e-10

$1.7010-10$

$1.0510-10$

$1.5620-10$

$1.4710-10$

$1.3650-10$

$1.2140-10$

$1.097 e-10$

$9.5400-11$

$7.8100-11$

$7.708 e-11$

$6.985 e-11$

$6.672 e-11$

$6.180 e-11$

5.373e-11

4.883e-11

$4.693 \mathrm{e}-11$

4.414e-11

$3.937 e-11$

3.269e-11

2.916e-11

2.732e-11

$2.483 e-11$

2.231e-11

2.140e-11

2.045e-11

$1.947 e-11$

$1.850 e-11$

$1,762 e-11$
7.120-10

6. $1120-10$

6. $5 \times 70-10$

6.346-10

0.2110-10

6.chice-10

3.9440-10

$5.7970-10$

5.575e-10

4. $000-10$

$3.3020-10$

2.737e-10

2. $430 e-10$

2.145e-10

1.755e-10

1.655e-10

$1.565 e-10$

$1.4746-10$

1. 369e-10

1.217e-20

1. 100 e-10

9.563e-11

7. $8310-11$

7.715e-11

6.990e-11

6.677e-11

6.184e-11

$5.377 e-11$

4.886e-11

$4.696 e-11$

4.416e-11

3.940e-11

3. 271e-11

2.928e-11

2.734e-11

2. 485e-11

2. 232e-11

2. 141e-11

2. 046e-11

$1.948 e-11$

$1.850 \mathrm{e}-11$

$1.763 \mathrm{e}-11$

Page -37- 
croy

$7.2500-01$

7. $716 \mathrm{cos}$

7.940 -

0. Lem.03

0.390.-3

0.cue-03

0. nume.03

$9.2000-03$

-.4000-03

-.5600-03

0.000-03

1.0000-02

1.040-02

1.004- -02

1.1100-02

1.150e-02

$1.270-02$

1.300e-02

1.3900-02

1.470 - 02

1.520e-02

1. 570e-02

1.640e-02

1.700e-02

1.720e-02

1. 110e-02

1.900e-02

2.970e-02

2.010e-02

2.100e-02

2.170e-02

2.230e-02

2.310e-02

2.550e-02

2.920e-02

3.320e-02

3. $750 e-02$

4. 200e-02

4.740e-02

4.850e-02

5. 020e-02
Enet

1. $180-11$

$2.576-12$

2. 35e-12

1.216-21

1.2760-11

1. $1350-11$

$1.0720-11$

1.008e-11

9.506- 12

$0.027 e-12$

c. $458-12$

$7.0120-22$

$7.4300-12$

$7.259 e-12$

7.000- -12

6.760e-12

6. 1350-12

5.643e-12

5.019e-12

4.010e-12

3.186e-12

2.986e-12

2.866e-12

2.717e-12

2.615e-12

$2.459 e-12$

2.175e-12

$1.890 \mathrm{e}-12$

1.681e-12

1.616e-12

1.581e-12

$1.547 e-12$

1. 505e-12

1. $384 e-12$

1. 074e-12

7.176e-13

$6.178 e-13$

$4.908 e-13$

4. $360 e-13$

4.048e-13

$3.882 e-13$ coustion

6. $0410-12$

6. 10. -12

$5.175 \cdot 12$

4. (46)-12

4. 472e-12

4. 315e-12

$4.057 e-12$

$3.7960-12$

3.506e-12

3.397e-12

3.1750-12

2. $9100-12$

2.790-12

2.703e-12

$2.6350-12$

2.505e-12

2.276e-12

2.089e-12

$1.052 e-12$

1. $4700-12$

1.15Be-12

$1.0840-12$

$1.042 e-12$

$9.882 e-13$

9.519e-13

$8.963 e-13$

$7.946 e-23$

$6.923 e-13$

$6.172 e-13$

$5.953 e-13$

5. $850 e-13$

5.747e-13

5. $616 e-13$

5. $234 e-13$

4. 218e-13

3. $068 \mathrm{e}-13$

2. 839e-13

2. $524 e-13$

2. $471 e-13$

2. $452 e-13$

2. $433 e-13$ er alt bater sat. alt

$1.020-11$

$1.520-11$

1. 3m20-11

$1.176-11$

1. 136-11

1.0se-11

1.030 -11

$9.712 e+12$

$9.213 e-12$

0.755e-12

$0.2150-12$

7. $590-12$

$7.222 e-12$

$7.057 e-12$

$6.9850-12$

6.557e-12

5. $973 e-12$

$5.497 e-12$

4.094e-12

3.919e-12

3.122e-12

2.929e-12

2.811e-12

2.666e-12

$2.566 e-12$

2.414e-..2

$2.137 \mathrm{e}-12$

$1.859 e-12$

$1.655 e-12$

1.591e-12

$1.556 e-12$

1. 523e-12

1. 481e-12

1. 362e-12

1.056e-12

7.041e-13

$6.037 e-13$

$4.762 e-13$

$4.199 e-13$

3. $875 e-13$

3. $705 e-13$
1. c.5. 14

1.5210.11

1. $3020-11$

1. 11) 12

1. ISE- 14

1.ene-11

1. 032e-11

- .7210-12

9.216-12

0.757e-12

$0.217 e-12$

$7.590=-12$

7.223e-12

7.054- -12

$6.007 e-12$

6.559e-12

5.974e-12

5. $498 \mathrm{e}-12$

4.895e-12

3.919e-12

3.122e-12

2.929e-12

2.812e-12

2.666e-12

$2.566 e-12$

2. 415e-12

2. 137e-12

1.859e-12

1. 655e-12

1. $591 e-12$

1. 556e-12

1. 523e-12

1. 481e -12

1. $362 e-12$

1.056e-12

7. 042e-13

$6.038 e-13$

4.764e-13

4. 201e-13

3.877e-13

3.707e-13

Page -38- 

Dary - in ?

s.sen-e: c.10me-1. c.7there c.senele 7.3500 7. exea-h - eree-ce - $390 \mathrm{ser}$ C.c00-02 -.050e-02 9.2300-02 9.600e-02 2.000e-01 1.050e-01 1.1000-01 1. 150e-01 1.210e-01 1.350e-01 1.490e-01 1.640e-01 1.800e-01 1.980e-01 2.180e-01 2.390e-01

2.630e-01

2.900e-01

3.190e-01

3.510a-01

3.860e-01 4.240e-01 4.670e-01 5.130e-01 5.650e-01 6. $210 \mathrm{e}-01$ 6.830e-01 7. 510e-01 B. 260e-01 $9.090 e-01$ $1.000 e+00$ 1. $120 \mathrm{e}+00$ $1.270 \mathrm{e}+00$ $1.420 \mathrm{e}+00$

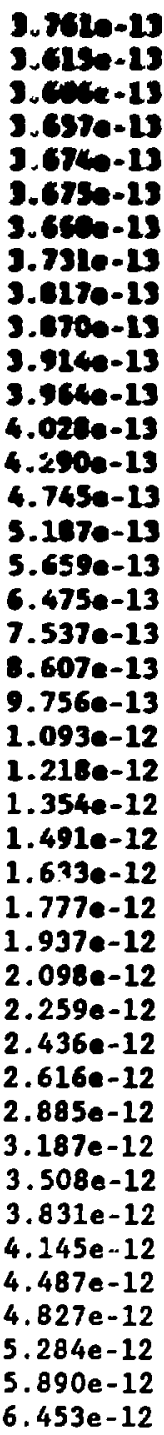

3. $2010-13$

c15e- 13

357e-15

3.675-12

3.675-13

3.640.28

$3.7310-13$

$3.0170-13$

$3.070-18$

$3.916-13$

$3.9640-13$

$4.0240-13$

$4.2500-13$

$4.7450-13$

$5.1870-13$

5.659e-13

$6.475 e-13$

$7.537 e-13$

$8.607 e-13$

1.218e-12

$1.354-12$

$1.6230-12$

$1.777 e-12$

.937e-12

$2.259 \cdot-12$

2.436e-12

.616e-12

3. 187e-12

3. $508 e-12$

$3.831 e-12$

$4.145 e-12$

$4.487 e-12$

$4.827 e-12$

5. $890 e-12$

$6.453 e-12$

merrien

Cos atse

Gove sec. vit

2. $480-23$

2.5ecto- 13

2. $716-13$

2. $0330-13$

2. $420-13$

$3.0220-13$

3.09se-23

3.1910-13

3.300- -23

$3.3720-13$

3.432e- 23

3.507e-13

3. $607 a-13$

3. $1070-13$

4.3340-13

$4.776-13$

$5.247 e-13$

6.05e-13

$7.152 e-13$

8.230e-13

9.356e-13

1.051e-12

1.174e-12

1.306e-12

1. 439 e- 12

$1.578-12$

1.719e-12

$1.874 e-12$

2. 030e-12

2.186e-12

2.358e-12

2.532e-12

2.7946-12

3. 086a-12

3. 397e-12

3. $710 e-12$

4. $014 e-12$

4. $345 e-12$

4.675e-12

5.118e-12

5. $704 e-12$

6. $250 e-12$
$3.5720-23$

3.c5-23

3.3710-13

3. 4ce-13

$3.428-13$

$3.3370-13$

$3.358-15$

3.4270-13

3.5010-13

3.5460-13

3.5020-13

3.623e-13

3.6740-13

3. 505 e -13

4.313a-13

4.705e-13

5.1300-13

5.85se-13

6.004e-13

7.759e-13

$0.709 e-13$

$9.837 e-13$

1.097e-12

1. 218e-12

1.342e-12

1. $459 a-12$

1.599e-12

$1.743 e-12$

$1.887 e-12$

2.031e-12

2.191e-12

2.353e-12

2.595e-12

2. 866e-12

3.1.55e-12

3. $4456-12$

3.727e-12

4.035e-12

4. 341e-12

4. 752e-12

5. 296e-12

$5.804 e-12$
$3.576-21$

3.ce-13

$3.3760-25$

3.40-2) 23

$3.416-13$

J. $4010-13$

3.3730-13

3. $4320-13$

3.5050-13

3.5500-13

3.5xce-13

3.620-13

3.679a-13

3.9110-13

$4.3196-13$

$4.715 e-13$

5.13Le-13

5.067e-13

6.815e-13

7.771e-13

6.803e-13

9.853e-13

1.099e-12

1.220e-12

$1.344 a-12$

1. $4710-12$

1.601e-12

1. 745e-12

$1.890 e-12$

2.034e-12

2. 194e-12

2. 356e-12

2.599e-12

2.87le-12

$3.160 e-12$

$3.451 e-12$

$3.733 \mathrm{e}-12$

4. $041 \mathrm{e}-12$

4. 347e-12

4. $759 e-12$

5. $305 e-12$

$5.813 e-12$

Page - 39 - 
Cis

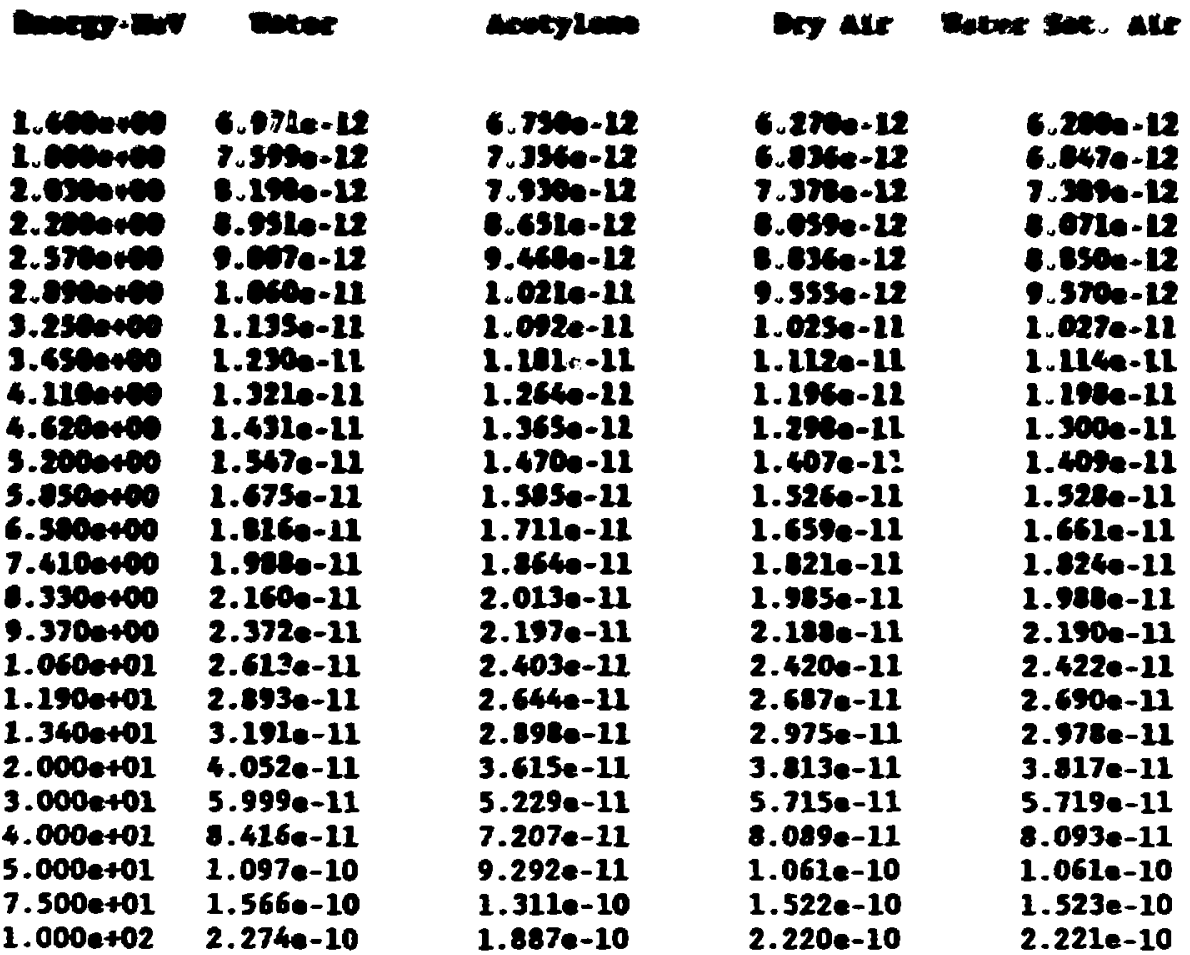


$a x$

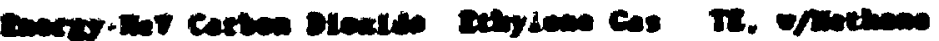

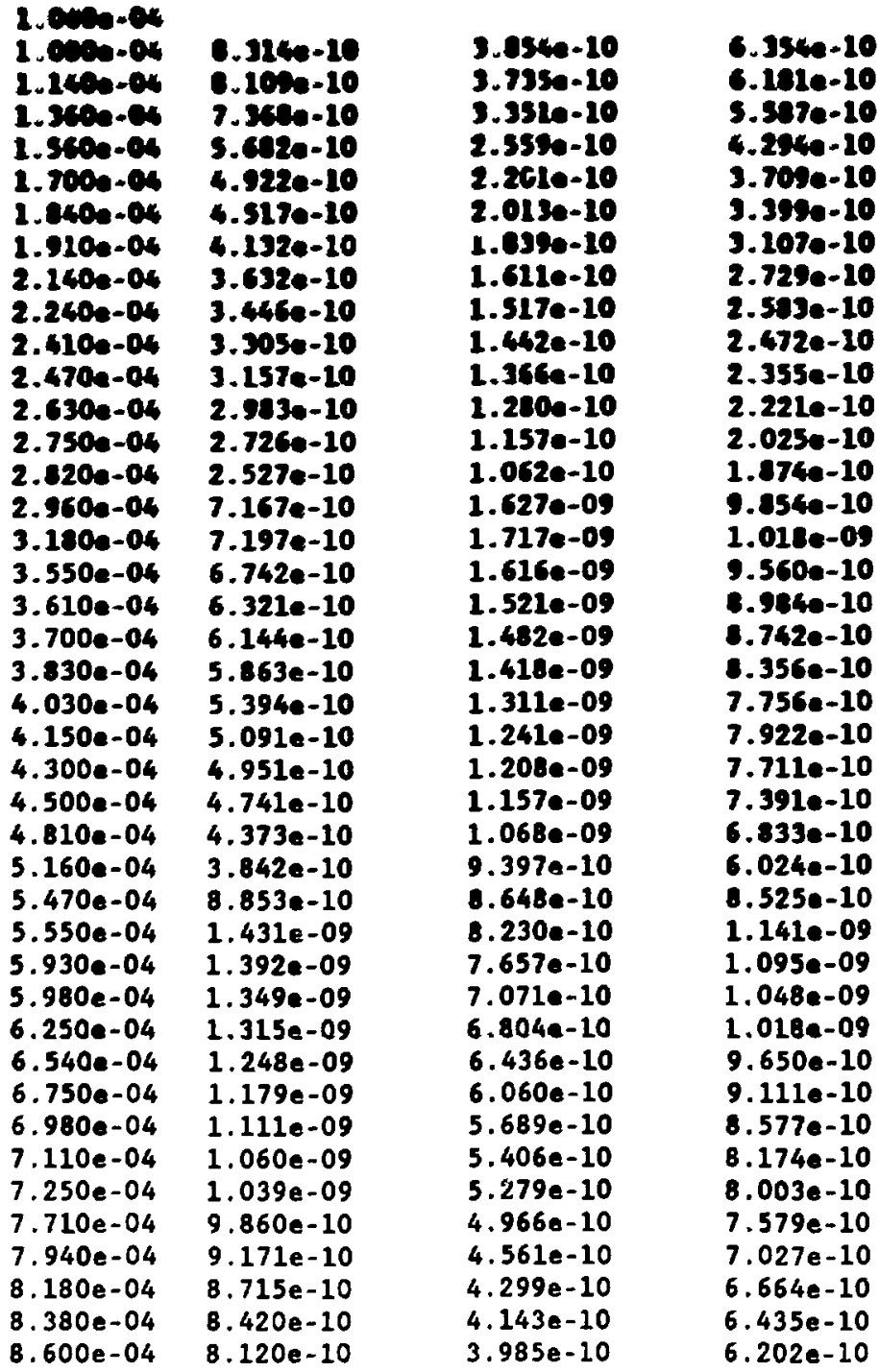

Page -41- 


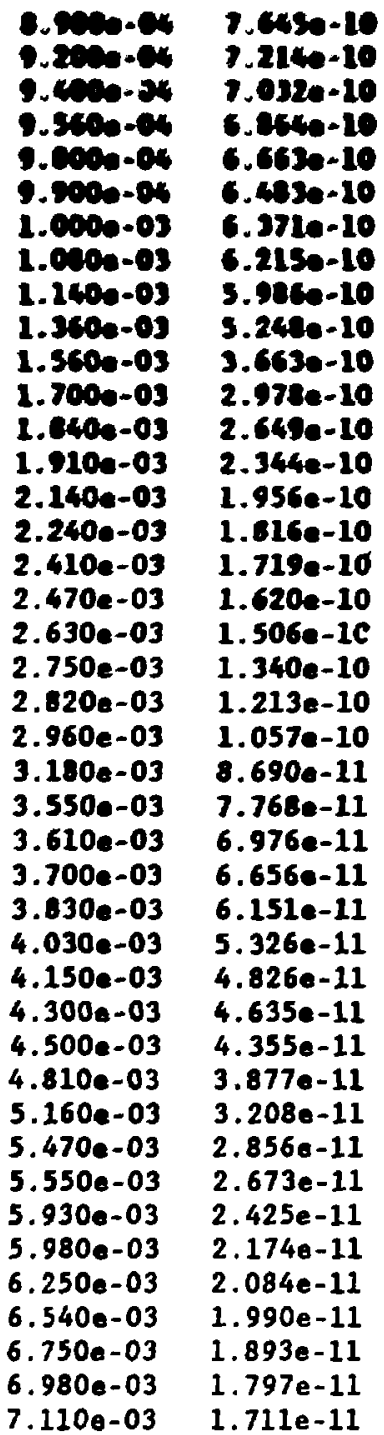

1. $7 x-10$

$3.50 s-10$

3. cate-10

3. 316-10

$3.206-10$

$3.107 e-10$

$3.047 e-20$

2. $9640-10$

2. $0470-10$

2.6760 .10

1. $6960-10$

$1.3490-10$

1. $1920-10$

$1.0460-10$

c.623e-11

$7.966 e-11$

7.521e-11

7.066e-11

$6.547 e-11$

5.791e-11

5.212e-11

4.504e-11

3.652e-11

3. 246e-11

2. $900 e-11$

2.761e-11

2.541e-11

2.181e-11

1. 965e-11

$1.884 e-11$

$1.765 e-11$

1.563e-11

1. 280e-11

1.133e-11

1.057e-11

9. $545 e-12$

8. 508e-12

8. $140 \mathrm{e}-12$

$7.758 \mathrm{e}-12$

$7.367 e-12$

$6.977 \mathrm{e}-12$

$6.630 e-12$
5.036-10

5. $4940-10$

3. 359-10

5.2240-10

S.057e-10

$4.9230-20$

4.03he-10

4.717e-10

4.3400-10

$3.9730-10$

$2.756-10$

2.233--10

1. $942 e-10$

1.7510-10

$1.457 e-10$

1.351e-10

1. 278e-10

1.204e-10

1.118e-10

$9.939 e-11$

c.985e-11

$7.816 e-11$

6. $404 e-11$

5.718e-11

5.129e-11

4.891e-11

4.516e-11

3.903e-11

3.532e-11

3. 391 $a-11$

3. $184 a-11$

2.832e-11

$2.338 e-11$

2.079e-11

$1.944 e-11$

1. $762 e-11$

$1.578 e-11$

1. $512 e-11$

$1.443 e-11$

1. $373 e-11$

1. $302 e-11$

1. $239 e-11$

Page - 42 - 


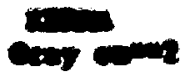

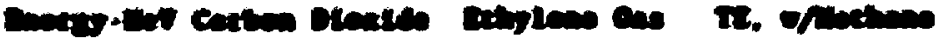

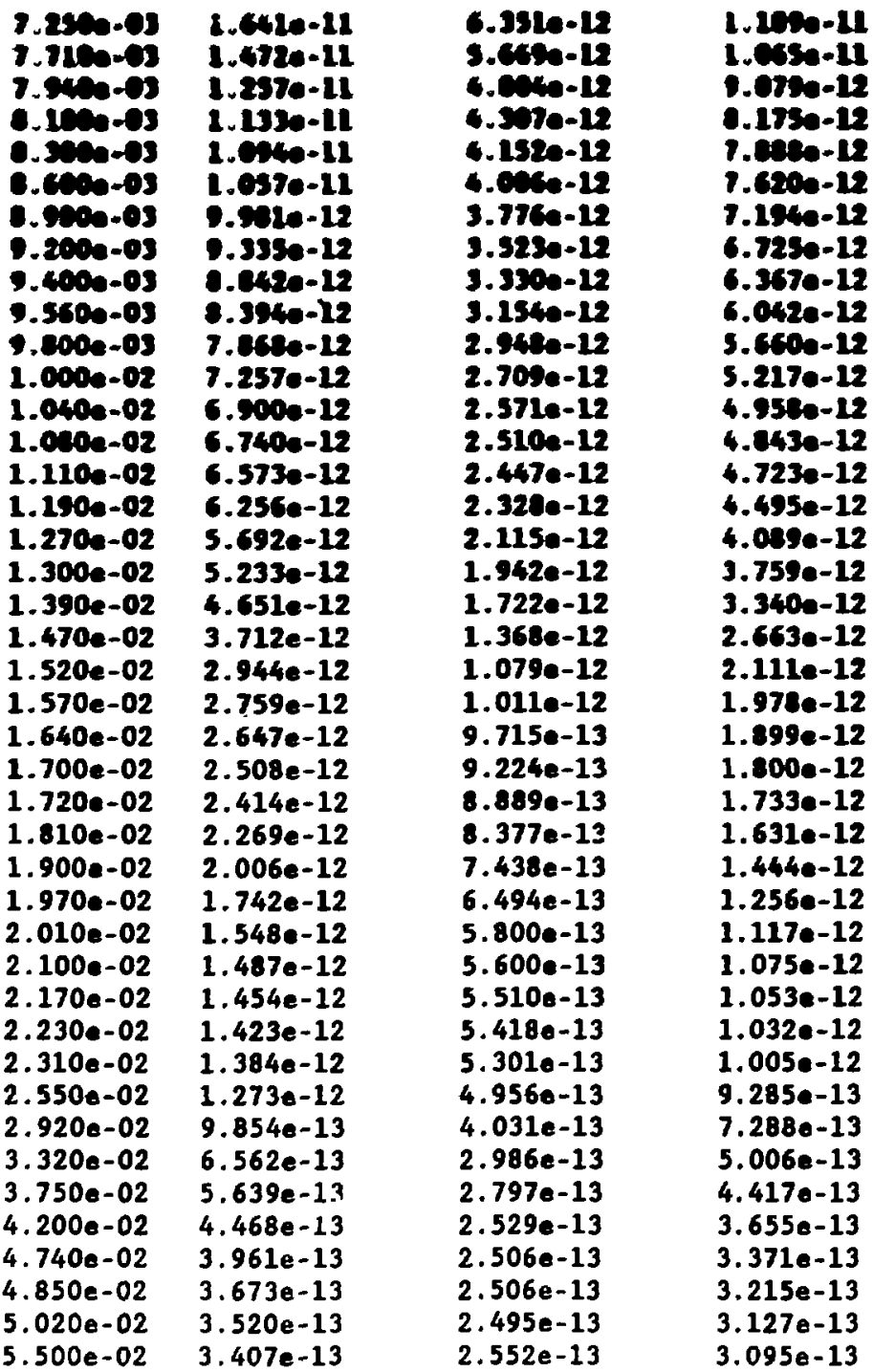

Page -43- 


\begin{tabular}{|c|c|c|c|}
\hline $\begin{array}{l}01 \\
01 \\
01 \\
01 \\
01 \\
01 \\
01 \\
01 \\
01 \\
01 \\
01 \\
01 \\
01 \\
01 \\
01 \\
01 \\
01 \\
01 \\
01 \\
01 \\
01 \\
01 \\
01 \\
01 \\
01\end{array}$ & $\begin{array}{l}3.211 e-11 \\
3.259 e-13 \\
3.393 e-13 \\
3.31 e e-13 \\
3.317 e-13 \\
3.392 e-13 \\
3.36 e-13 \\
3.443 e-13 \\
3.491 e-13 \\
3.530 e-13 \\
3.575 e-13 \\
3.632 e-13 \\
3.867 e-13 \\
4.276 e-23 \\
4.675 e-13 \\
5.099 e-13 \\
5.033 e-13 \\
6.789 e-13 \\
7.752 e-13 \\
8.786 e-13 \\
9.838 e-13 \\
1.097 e-12 \\
1.219 e-12 \\
1.343 e-12 \\
1.471 e-12 \\
1.601 e-12 \\
1.745 e-12 \\
1.889 e-12 \\
2.034 e-12 \\
2.194 e-12 \\
2.356 e-12 \\
2.599 e-12 \\
2.870 e-12 \\
3.159 e-12 \\
3.450 e-12 \\
3.732 e-12 \\
4.040 e-12 \\
4.347 e-12 \\
4.759 e-12 \\
5.304 e-12 \\
5.812 e-12 \\
6.279 e-12\end{array}$ & $\begin{array}{l}2.65 e-13 \\
2.030 e-13 \\
2.961 e-13 \\
3.045 e-13 \\
3.161 e-13 \\
3.246 e-13 \\
3.359 e-13 \\
3.476 e-13 \\
3.553 e-13 \\
3.616 e-13 \\
3.699 e-13 \\
3.008 e-13 \\
4.106 e-13 \\
4.584 e-13 \\
5.051 e-13 \\
5.551 e-13 \\
6.425 e-13 \\
7.578 e-13 \\
8.724 e-13 \\
9.919 e-13 \\
1.114 e-12 \\
1.245 e-12 \\
1.385 e-12 \\
1.527 e-12 \\
1.673 e-12 \\
1.823 e-12 \\
1.988 e-12 \\
2.153 e-12 \\
2.318 e-12 \\
2.501 e-12 \\
2.686 e-12 \\
2.963 e-12 \\
3.273 e-12 \\
3.603 e-12 \\
3.935 e-12 \\
4.257 e-12 \\
4.609 e-12 \\
4.959 e-12 \\
5.428 e-12 \\
6.050 e-12 \\
6.629 e-12 \\
7.159 e-12\end{array}$ & $\begin{array}{l}3.074 e-13 \\
3.151 e-13 \\
3.239 e-13 \\
3.206 e-13 \\
3.345 e-13 \\
3.379 e-13 \\
3.46 e-13 \\
3.567 e-13 \\
3.631 e-13 \\
3.603 e-13 \\
3.747 e-13 \\
3.432 e-13 \\
4.105 e-13 \\
4.561 e-13 \\
5.005 e-13 \\
5.440 e-13 \\
6.305 e-13 \\
7.388 e-13 \\
3.470 e-13 \\
9.615 e-13 \\
1.078 e-12 \\
1.204 e-12 \\
1.338 e-12 \\
1.475 e-12 \\
1.616 e-12 \\
1.759 e-12 \\
1.918 e-12 \\
2.077 e-12 \\
2.237 e-12 \\
2.413 e-12 \\
2.591 e-12 \\
2.858 e-12 \\
3.157 e-12 \\
3.475 e-12 \\
3.795 e-12 \\
4.106 e-12 \\
4.445 e-12 \\
4.782 e-12 \\
5.235 e-12 \\
5.835 e-12 \\
6.394 e-12 \\
6.906 e-12\end{array}$ \\
\hline
\end{tabular}

Page -44- 


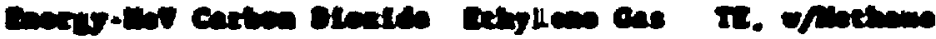

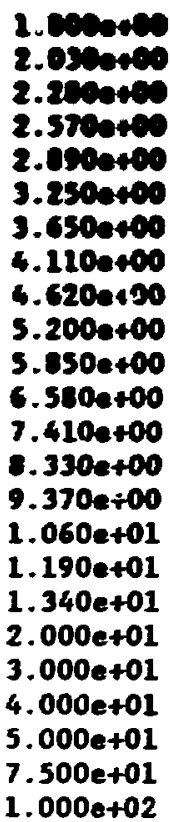

$0.046-12$
$7.390 e-12$
$1.071 e-12$
$1.096 e-12$
$9.572 e-12$
$1.027 e-11$
$1.115 e-11$
$1.199 e-11$
$1.301 e-11$
$1.411 e-11$
$1.531 e-11$
$1.665 e-11$
$1.026 e-11$
$1.993 e-11$
$2.198 e-11$
$2.432 e-11$
$2.702 e-11$
$2.992 e-11$
$3.840 e-11$
$5.762 e-11$
$8.164 e-11$
$1.071 e-10$
$1.538 e-10$
$2.245 e-10$

7.c01e-12

$7.527 e-12$

0.c01e-12

$0.2010-12$

1.0030-11

1.08310-11

1.15 se-11

$1.2410-11$

$1.3340-11$

$1.438--11$

$1.3470-11$

$1.655 e-11$

$1.7^{2} 4 e-11$

-. $1170-12$

-. es:-12

0.605e-12

1.067e-11

2. 1210-11

1.2130-11

1.300e-11

1. $4050-11$

1.516e-11

1.630e-11

1.771e-11

$1.9,1 e-11$

1. $934 e-11$

2.10:1e-11

2.094e-11

2.286e-11

2.291e-11

2. $45.2 e-11$

2.514e-11

2.736e-11

2.774e-11

2. $9900-11$

3.050e-11

$3.698-11$

3.834e-11

5.605e-11

7. 788e-11

1.009e-10

1. $4320-10$

2.069e-10 


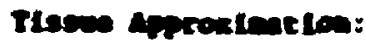

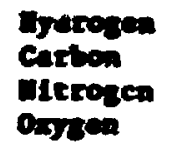

.1002

.1493

.0346

.7157

Lome (Pener):

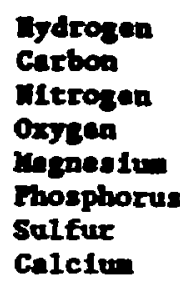

.0640

.2700

.0270

.4100

.0020

.0700

.0020

.1470

Huscle (ICRU):

$\begin{array}{ll}\text { Hydrogen } & .1020 \\ \text { Carbon } & .1230 \\ \text { MItrogen } & .0350 \\ \text { Orggen } & .7290 \\ \text { Sodiun } & .0008 \\ \text { Yagnesium } & .0002 \\ \text { Phosphorus } & .0020 \\ \text { Sulfux } & .0050 \\ \text { Potase1ism } & .0030 \\ \text { Calcium } & .0001\end{array}$

Standard Man :

$\begin{array}{ll}\text { Hydrogen } & .1000 \\ \text { Carbon } & .1800 \\ \text { Mitrogen } & .0300 \\ \text { Oxygen } & .6500 \\ \text { Sodiun } & .0015 \\ \text { Magnesium } & .0005 \\ \text { Phosphorus } & .0100 \\ \text { Sulfur } & .0025 \\ \text { Chlorine } & .0015 \\ \text { Potassium } & .0020 \\ \text { Calcium } & .0150 \\ \text { Iron } & .0070\end{array}$


A.ts trentic:

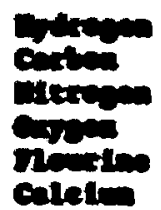

.tots

-77x*

cose

$-632$

.0174

.0154

byten rye oc ors:

Iyleogen

certen

niterogen

ansenen

. $\cos$

.1230

.1414

Ineles:

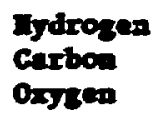

.0905

.5938

.3196

Liquid, Huscle Equivalent:

$$
\begin{aligned}
& \text { Hjdrogen } \\
& \text { Curbon } \\
& \text { ultrogen } \\
& \text { Oxgen }
\end{aligned}
$$

.1020

.1201

.0354

.7425

Water:

Hydrogen

.1119

Oxgren

.8881

Acetylene:

Hydrogen

.0774

Carbon

.9226

Air, Dry:

Garbon
Nitrogen
Oxygen
Argon

.0001

.7552

.2318

.0129

Page - 47- 


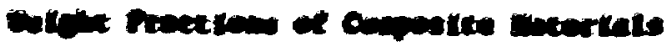

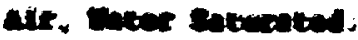

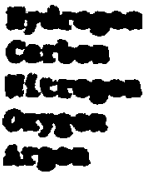

CORC

cent

.743

.2413

.0127

Caxter Dientle:

$$
\text { cartien }
$$

Ixishing:

$$
\text { iblinosen }
$$

carboa

TE Ges, wth Wathane:

$$
\begin{aligned}
& \text { Bjdrozen } \\
& \text { Carbon } \\
& \text { mitrozen } \\
& \text { oxysen }
\end{aligned}
$$

.0352

.4068 